\title{
GLOBAL WELL-POSEDNESS OF THE PERIODIC CUBIC FOURTH ORDER NLS IN NEGATIVE SOBOLEV SPACES
}

\author{
TADAHIRO $\mathrm{OH}^{1}$ and YUZHAO WANG ${ }^{1,2}$ \\ ${ }^{1}$ School of Mathematics, The University of Edinburgh, and The Maxwell Institute for the \\ Mathematical Sciences, James Clerk Maxwell Building, The King's Buildings, Peter Guthrie Tait \\ Road, Edinburgh EH9 3FD, UK; \\ email: hiro.oh@ed.ac.uk \\ ${ }^{2}$ School of Mathematics, Watson Building University of Birmingham, Edgbaston, \\ Birmingham B15 2TT, UK; \\ email: y.wang.14@bham.ac.uk
}

Received 19 June 2017; accepted 22 March 2018

\begin{abstract}
We consider the Cauchy problem for the cubic fourth order nonlinear Schrödinger equation (4NLS) on the circle. In particular, we prove global well-posedness of the renormalized 4NLS in negative Sobolev spaces $H^{s}(\mathbb{T}), s>-\frac{1}{3}$, with enhanced uniqueness. The proof consists of two separate arguments. (i) We first prove global existence in $H^{s}(\mathbb{T}), s>-\frac{9}{20}$, via the short-time Fourier restriction norm method. By following the argument in Guo-Oh for the cubic NLS, this also leads to nonexistence of solutions for the (nonrenormalized) 4NLS in negative Sobolev spaces. (ii) We then prove enhanced uniqueness in $H^{s}(\mathbb{T}), s>-\frac{1}{3}$, by establishing an energy estimate for the difference of two solutions with the same initial condition. For this purpose, we perform an infinite iteration of normal form reductions on the $H^{s}$-energy functional, allowing us to introduce an infinite sequence of correction terms to the $H^{s}$-energy functional in the spirit of the $I$-method. In fact, the main novelty of this paper is this reduction of the $H^{s}$-energy functionals (for a single solution and for the difference of two solutions with the same initial condition) to sums of infinite series of multilinear terms of increasing degrees.
\end{abstract}

2010 Mathematics Subject Classification: 35Q55

\section{Introduction}

\subsection{The cubic nonlinear Schrödinger equation with quartic dispersion. In} this paper, we consider the Cauchy problem for the cubic fourth order nonlinear

(C) The Author(s) 2018. This is an Open Access article, distributed under the terms of the Creative Commons Attribution licence (http://creativecommons.org/licenses/by/4.0/), which permits unrestricted re-use, distribution, and reproduction in any medium, provided the original work is properly cited. 
Schrödinger equation (4NLS) on the circle $\mathbb{T}=\mathbb{R} /(2 \pi \mathbb{Z})$ :

$$
\left\{\begin{array}{l}
i \partial_{t} u=\partial_{x}^{4} u \pm|u|^{2} u \\
\left.u\right|_{t=0}=u_{0},
\end{array} \quad(x, t) \in \mathbb{T} \times \mathbb{R},\right.
$$

where $u$ is a complex-valued function. The equation (1.1) is also called the biharmonic nonlinear Schrödinger equation (NLS) and it was studied in [22, 40] in the context of stability of solitons in magnetic materials. See also [2, 16, 23, 24] for a more general class of fourth order NLS:

$$
i \partial_{t} u=\lambda \partial_{x}^{2} u+\mu \partial_{x}^{4} u \pm|u|^{2} u .
$$

In the following, we focus our attention on the equation (1.1). See Remark 1.8 for a brief discussion on (1.2).

Our main goal is to study the well/ill-posedness issue of (1.1) in the low regularity setting. We first recall the scaling symmetry for (1.1); if $u(x, t)$ is a solution to (1.1) on $\mathbb{R}$, then $u_{\lambda}(x, t)=\lambda^{-2} u\left(\lambda^{-1} x, \lambda^{-4} t\right)$ is also a solution to (1.1) on $\mathbb{R}$ with the scaled initial data $u_{0, \lambda}(x)=\lambda^{-2} u_{0}\left(\lambda^{-1} x\right)$. This scaling symmetry induces the so-called scaling critical Sobolev regularity $s_{\text {crit }}:=-\frac{3}{2}$, leaving the homogeneous $\dot{H}^{s_{\text {crit }}}$-norm invariant under the scaling symmetry. On the one hand, the scaling argument provides heuristics indicating that a PDE is well-posed in $H^{s}$ for $s \geqslant s_{\text {crit }}$ and is ill-posed in $H^{s}$ for $s<s_{\text {crit }}$. This heuristics certainly applies to many equations, including NLS and the nonlinear wave equations. See [9]. On the other hand, this heuristics is known to often fail in negative Sobolev spaces. This is indeed the case for (1.1) and its renormalized variant (1.5).

In [36], the first author and Tzvetkov proved that (1.1) is globally well-posed in $H^{s}(\mathbb{T})$ for $s \geqslant 0$. The proof is based on the Fourier restriction norm method (namely, utilizing the $X^{s, b}$-space defined in (2.7)) with the $L^{4}$-Strichartz estimate:

$$
\|u\|_{L_{x, t}^{4}} \lesssim\|u\|_{X^{0,5 / 16}}
$$

along with the conservation of the $L^{2}$-norm. Following the approach in [5, 8], it was also shown in [36] that (1.1) is mildly ill-posed in $H^{s}(\mathbb{T}), s<0$, in the sense that the solution map: $u_{0} \in H^{s}(\mathbb{T}) \mapsto u \in C\left([-T, T] ; H^{s}(\mathbb{T})\right)$ is not locally uniformly continuous for $s<0$. Moreover, following the work [19], it was pointed out in [36] that (1.1) is indeed ill-posed in negative Sobolev spaces by establishing a nonexistence result. See Corollary 1.2 below for a precise statement. We also mention the following norm inflation result due to Choffrut and Pocovnicu [6]. Let $s<-\frac{7}{6}$. Then, given any $\varepsilon>0$, there exist a solution $u_{\varepsilon}$ to (1.1) on $\mathbb{T}$ and $t_{\varepsilon} \in(0, \varepsilon)$ such that

$$
\left\|u_{\varepsilon}(0)\right\|_{H^{s}(\mathbb{T})}<\varepsilon \quad \text { and } \quad\left\|u_{\varepsilon}\left(t_{\varepsilon}\right)\right\|_{H^{s}(\mathbb{T})}>\varepsilon^{-1} .
$$


See also [38]. It is worthwhile to note that the regularity $-\frac{7}{6}$ is higher than the scaling critical regularity $s_{\text {crit }}=-\frac{3}{2}$ and that this norm inflation result for $s<-\frac{7}{6}$ also applies to the renormalized 4NLS (1.5) below.

In the next subsection, we introduce an alternative formulation for (1.1) such that (i) it is equivalent to (1.1) in $L^{2}(\mathbb{T})$ but (ii) it behaves better than (1.1) in negative Sobolev spaces. In the following, the defocusing/focusing nature of the equation (1.1) does not play any role. Hence, we assume that it is defocusing, that is, with the + sign in (1.1).

1.2. Renormalized cubic fourth order NLS. Given a global solution $u \in$ $C\left(\mathbb{R} ; L^{2}(\mathbb{T})\right)$ to (1.1), we define the following invertible gauge transformation $\mathcal{G}$ by

$$
\mathcal{G}(u)(t):=e^{2 i t \mu(u)} u(t)
$$

with its inverse

$$
\mathcal{G}^{-1}(u)(t):=e^{-2 i t \mu(u)} u(t),
$$

where $\mu(u)=f|u(x, t)|^{2} d x:=(1 / 2 \pi) \int_{\mathbb{T}}|u(x, t)|^{2} d x$. Thanks to the $L^{2}$ conservation, $\mu(u)$ is defined, independently of $t \in \mathbb{R}$, as long as $u_{0} \in L^{2}(\mathbb{T})$. A direct computation shows that the gauged function, which we still denote by $u$, satisfies the following renormalized 4NLS:

$$
\left\{\begin{array}{l}
i \partial_{t} u=\partial_{x}^{4} u+\left(|u|^{2}-2 f|u|^{2} d x\right) u \quad(x, t) \in \mathbb{T} \times \mathbb{R} . \\
\left.u\right|_{t=0}=u_{0},
\end{array}\right.
$$

This renormalization appears as an equivalent formulation of the Wick renormalization in Euclidean quantum field theory [4, 34, 35]. (By viewing $u$ as a complex-valued Gaussian random variables, the Wick renormalization of $|u|^{2} u$ is nothing but a projection onto the Wiener homogeneous chaoses of order three.) For this reason, we will refer to (1.5) as the Wick ordered cubic $4 N L S$ in the following.

In view of the invertibility of $\mathcal{G}$ on $L^{2}(\mathbb{T})$, we see that the original cubic 4NLS (1.1) and the Wick ordered cubic 4NLS (1.5) describe equivalent dynamics on $L^{2}(\mathbb{T})$. On the other hand, the gauge transformation $\mathcal{G}$ does not make sense outside $L^{2}(\mathbb{T})$. Hence, they describe genuinely different dynamics, if any, outside $L^{2}(\mathbb{T})$.

It is easy to see that this specific choice of gauge for (1.5) removes a certain singular component from the cubic nonlinearity. Indeed, the nonlinearity on the right-hand side of (1.5) can be written as

$$
\mathfrak{N}(u)=\mathfrak{N}(u, u, u):=\left(|u|^{2}-2 f|u|^{2} d x\right) u
$$




$$
=\mathcal{N}(u, u, u)-\mathcal{R}(u, u, u),
$$

where the nonresonant part $\mathcal{N}$ and the resonant part $\mathcal{R}$ are defined by

$$
\begin{aligned}
& \mathcal{N}\left(u_{1}, u_{2}, u_{3}\right)(x, t)=\sum_{n \in \mathbb{Z}} \sum_{\substack{n=n_{1}-n_{2}+n_{3} \\
n \neq n_{1}, n_{3}}} \widehat{u}_{1}\left(n_{1}, t\right) \overline{\widehat{u}_{2}\left(n_{2}, t\right)} \widehat{u}_{3}\left(n_{3}, t\right) e^{i n x}, \\
& \mathcal{R}\left(u_{1}, u_{2}, u_{3}\right)(x, t)=\sum_{n \in \mathbb{Z}} \widehat{u}_{1}(n, t){\widehat{\widehat{u}_{2}}}_{2}(n, t) \widehat{u}_{3}(n, t) e^{i n x} .
\end{aligned}
$$

Namely, the gauge transformation basically eliminates the contribution from $n=$ $n_{1}$ or $n=n_{3}$. In the following, we choose to study the Wick ordered cubic 4NLS (1.5). As with any renormalization procedure or gauge choice, we stress that this is a matter of choice. See Remark 1.3.

We now state our first result.

THEOREM 1.1 (Global existence). Let $s \in\left(-\frac{9}{20}, 0\right)$. Given $u_{0} \in H^{s}(\mathbb{T})$, there exists a global solution $u \in C\left(\mathbb{R} ; H^{s}(\mathbb{T})\right)$ to the Wick ordered cubic $4 N L S$ (1.5) with $\left.u\right|_{t=0}=u_{0}$.

On the one hand, as in [36], one can easily prove local well-posedness of (1.5) in $L^{2}(\mathbb{T})$ by a Picard iteration. On the other hand, it is easy to see that (1.5) is mildly ill-posed in negative Sobolev spaces in the sense of the failure of local uniform continuity of the solution map [15, 36]; see [15, Remark 1.4]. This in particular implies that one cannot use a Picard iteration to construct solutions to (1.5) in negative Sobolev spaces. We instead use a more robust energy method to construct solutions. More precisely, we use the short-time Fourier restriction norm method to prove Theorem 1.1. Here, the short-time Fourier restriction norm method simply means that we use dyadically defined $X^{s, b}$-type spaces with suitable localization in time, depending on the dyadic size of spatial frequencies. A precursor of this method appeared in the work of Koch and Tzvetkov [28], where localization in time was combined with the Strichartz norms. The shorttime Fourier restriction norm method has been very effective in establishing $a$ priori bounds on solutions in low regularity spaces (yielding even uniqueness in some cases), in particular, where a solution map is known to fail to be locally uniformly continuous. See $[10,17,21,25-27]$.

Given $T>0$, let $F^{s}(T) \subset C\left([-T, T] ; H^{s}(\mathbb{T})\right)$ denote the local-in-time version of the $X^{s, b}$-space adapted to appropriately chosen short-time scales and let $N^{s}(T)$ be its 'dual' space. See Section 2 for their precise definitions. In establishing the local existence part of Theorem 1.1, our main goal is to establish the following three estimates:

$$
\text { Linear estimate: } \quad\|u\|_{F^{s}(T)} \lesssim\|u\|_{E^{s}(T)}+\|\mathfrak{N}(u)\|_{N^{s}(T)},
$$


Nonlinear estimate: $\quad\|\mathfrak{N}(u)\|_{N^{s}(T)} \lesssim\|u\|_{F^{s}(T)}^{3}$,

Energy estimate: $\quad\|u\|_{E^{s}(T)}^{2} \lesssim\left\|u_{0}\right\|_{H^{s}}^{2}+\|u\|_{F^{s}(T)}^{4}$,

where $E^{s}(T) \approx L^{\infty}\left([-T, T] ; H^{s}(\mathbb{T})\right)$. These three estimates yield an a priori bound on (smooth) solutions in $H^{s}(\mathbb{T})$, which allows us to prove existence of local-in-time solutions (without uniqueness) by a compactness argument. As we see in the later sections, the short-time restriction adapted to the spatial dyadic scales allows us to gain extra modulation (that is, smoothing) in the resonant case. This in particular enables us to prove the trilinear estimate (1.10) below $L^{2}(\mathbb{T})$.

As for the global existence part, we employ the following $H_{M}^{s}$-norm adapted to the parameter $M \geqslant 1$ defined by

$$
\|f\|_{H_{M}^{s}}=\left\|\left(M^{2}+n^{2}\right)^{s / 2} \widehat{f}(n)\right\|_{\ell_{n}^{2}} .
$$

While the $H_{M}^{s}$-norm is equivalent to the standard $H^{s}$-norm, we have the following decay property when $s<0$ :

$$
\lim _{M \rightarrow \infty}\|f\|_{H_{M}^{s}}=0
$$

for all $f \in H^{s}(\mathbb{T})$. This allows us to reduce the problem to a small data theory in some appropriate sense. See Section 6.

As a corollary to the local-in-time a priori estimate established in the proof of Theorem 1.1 for solutions to the Wick ordered cubic 4NLS (1.5) (see Remark 6.4), we obtain the following nonexistence result for the original cubic 4NLS claimed above.

COROLlaRY 1.2. Let $s \in\left(-\frac{9}{20}, 0\right)$ and $u_{0} \in H^{s}(\mathbb{T}) \backslash L^{2}(\mathbb{T})$. Then, for any $T>0$, there exists no distributional solution $u \in C\left([-T, T] ; H^{s}(\mathbb{T})\right)$ to the cubic $4 N L S$ (1.1) such that

(i) $\left.u\right|_{t=0}=u_{0}$.

(ii) There exist smooth global solutions $\left\{u_{n}\right\}_{n \in \mathbb{N}}$ to (1.1) such that $u_{n} \rightarrow u$ in $C\left([-T, T] ; \mathcal{D}^{\prime}(\mathbb{T})\right)$ as $n \rightarrow \infty$.

In [19], the first author with Guo proved a similar nonexistence result for the standard cubic NLS:

$$
i \partial_{t} u=\partial_{x}^{2} u+|u|^{2} u
$$

in $H^{s}(\mathbb{T}), s>-\frac{1}{8}$, by first establishing an a priori estimate for solutions to the following Wick ordered cubic NLS:

$$
i \partial_{t} u=\partial_{x}^{2} u+\left(|u|^{2}-2 f|u|^{2} d x\right) u .
$$


The main idea of the proof is to exploit the fast oscillation in the phase of the inverse gauge transformation (1.4) and apply Riemann-Lebesgue lemma. See [19, Section 9] for details. Note that our assumption in Corollary 1.2 is slightly weaker than that in [19, Theorem 1.1], namely, the convergence in (ii) is assumed only in $C\left([-T, T] ; \mathcal{D}^{\prime}(\mathbb{T})\right)$ but that the same proof applies since the only ingredient needed from this assumption is the following convergence: $\left\langle u_{n}(\cdot, t), \phi(\cdot, t)\right\rangle_{L_{x}^{2}} \rightarrow$ $\langle u(\cdot, t), \phi(\cdot, t)\rangle_{L_{x}^{2}}$ for any test function $\phi \in \mathcal{D}(\mathbb{T} \times[-T, T])$.

REMARK 1.3. By introducing another gauge transformation $\mathcal{G}_{\gamma}(u)(t):=$ $e^{i \gamma t \mu(u)} u(t)$ with a parameter $\gamma \in \mathbb{R}$, we arrive at a different renormalized cubic 4NLS:

$$
\begin{aligned}
i \partial_{t} u & =\partial_{x}^{4} u+\left(|u|^{2}-\gamma f|u|^{2} d x\right) u \\
& =\partial_{x}^{4} u+\left(|u|^{2}-\gamma \cdot \infty\right) u .
\end{aligned}
$$

As it was mentioned in [19] in the context of the cubic NLS, it is crucial to subtract off the right amount of infinity in this renormalization procedure. It is easy to see that (1.1), (1.5), and (1.13) are all equivalent in $L^{2}(\mathbb{T})$. In negative Sobolev spaces, however, they are very different. In fact, the same nonexistence result in negative Sobolev spaces holds for (1.13) unless $\gamma=2$, which shows that ' $2 \cdot \infty$ ' is the right amount to subtract in the renormalization procedure.

REMARK 1.4. By applying our analysis with a parameter $M \geqslant 1$, we can extend the local existence result of the Wick ordered cubic NLS (1.12) in [19] to global existence (without uniqueness) in $H^{s}(\mathbb{T}), s>-\frac{1}{8}$.

Next, we turn our attention to the uniqueness issue of the solutions constructed in Theorem 1.1. The main source of difficulty lies in establishing an energy estimate for the difference of two solutions. The energy estimate (1.11) for a single solution follows from an argument analogous to the $I$-method (the method of almost conservation laws) [12, 13], which is ultimately based on the conservation of the $L^{2}$-norm for (1.5). The $L^{2}$-norm of the difference of two solutions, however, is not conserved under (1.5). Moreover, an estimate of the form:

$$
\|u-v\|_{E^{s}(T)}^{2} \lesssim\|u(0)-v(0)\|_{H^{s}}^{2}+\left(\|u\|_{F^{s}(T)}^{3}+\|v\|_{F^{s}(T)}^{3}\right)\|u-v\|_{F^{s}(T)}
$$


is false since it would imply smooth dependence on initial data and such smooth dependence is known to fail in negative Sobolev spaces $[15,36]$. In the following, we establish an energy estimate for the difference of two solutions with the same initial condition and thus prove uniqueness of solutions to the Wick ordered cubic 4NLS (1.5). Furthermore, our argument for proving uniqueness does not use any auxiliary function space (in particular, we do not use the short-time Fourier restriction norm method) and thus yields uniqueness in an enhanced sense.

THEOREM 1.5 (Global well-posedness with enhanced uniqueness). Let $s \in\left(-\frac{1}{3}\right.$, $0)$. Then, the Wick ordered cubic $4 N L S(1.5)$ is globally well-posed in $H^{s}(\mathbb{T})$. More precisely, the solution constructed in Theorem 1.1 is unique and the solution map is continuous. Here, the uniqueness holds in an enhanced sense; the solution constructed in Theorem 1.1 is unique among all the solutions in $C\left(\mathbb{R} ; H^{s}(\mathbb{T})\right.$ ) to (1.5) with the same initial data equipped with smooth approximating solutions.

Here, we implicitly assume that these solutions belong to various auxiliary functions spaces so that the cubic nonlinearity makes sense in some appropriate manner. The point is that we do not need to know which auxiliary function space each solution belongs to. Moreover, we assume that they satisfy the local-in-time estimate: $\|u\|_{C_{T} H^{s}} \lesssim\left\|u_{0}\right\|_{H^{s}}$ for some $T=T\left(\left\|u_{0}\right\|_{H^{s}}\right)>0$. See Remark 8.19.

Note that our enhanced uniqueness does not assert unconditional uniqueness in $C\left(\mathbb{R} ; H^{s}(\mathbb{T})\right.$ ), since we do assume that solutions with smooth approximating solutions have some extra regularity so that the cubic nonlinearity makes sense. (By slightly modifying the presentation in [18], one can easily prove unconditional uniqueness of (1.1) and (1.5) in $C\left(\mathbb{R} ; H^{s}(\mathbb{T})\right)$ for $s \geqslant \frac{1}{6}$. Clearly, the threshold $s \geqslant \frac{1}{6}$ is sharp in view of the embedding: $H^{1 / 6}(\mathbb{T}) \subset L^{3}(\mathbb{T}$ ) (in making sense of the cubic nonlinearity). Recall also the nonuniqueness result by Christ [7] of weak solutions in the extended sense in negative Sobolev spaces, where the nonlinearity is interpreted only as a limit of smooth nonlinearities.) Instead, our uniqueness statement should be interpreted as follows; given $u_{0} \in H^{s}(\mathbb{T})$, let $u$ be a solution to (1.5) with $\left.u\right|_{t=0}=u_{0}$ constructed in Theorem 1.1 via this particular version of the short-time Fourier restriction norm method. Suppose that $v$ is another solution to (1.5) with $\left.v\right|_{t=0}=u_{0}$ constructed by some other method, for example, by another version of the short-time Fourier restriction norm method or by an adaptation of Takaoka and Tsutsumi's argument [39] to (1.5), where the definition of the $X^{s, b}$-space incorporates the initial data. (In a recent paper [29], Kwak applied the ideas from [32, 39] and proved local wellposedness of (1.5) in $H^{s}(\mathbb{T})$ for $s \geqslant-\frac{1}{3}$. On the one hand, this result extends local well-posedness of (1.5) to the endpoint regularity $s=-\frac{1}{3}$. On the other hand, the uniqueness in [29] holds only in (a variant of) the $X^{s, b}$-space. When 
$s>-\frac{1}{3}$, the enhanced uniqueness in Theorem 1.5 allows us to conclude that the local-in-time solution constructed in [29] agrees with our solution constructed in Theorem 1.5 and hence is global. When $s=-\frac{1}{3}$, global well-posedness of (1.5) is open. We also mention an analogous work by Miyaji and Tsutsumi [30], prior to [29], on local well-posedness of the (renormalized) third order NLS in negative Sobolev spaces, based on a variant of the $X^{s, b}$-space incorporating initial data.) In general, we do not have a way to compare these solutions belonging to different resolution spaces. The enhanced uniqueness in Theorem 1.5, however, asserts that $u$ and $v$ must agree. It is in this sense that our uniqueness statement in Theorem 1.5 is enhanced since it allows us to compare solutions constructed by different methods. It seems that this notion of enhanced uniqueness is one of the strongest forms of uniqueness 'in practice'. (Given a solution, it seems reasonable in practice to assume that it comes with at least one sequence of smooth approximating solutions.)

We stress that this enhanced uniqueness is by no means automatic since we do not have a priori continuous dependence. (Our proof of continuous dependence follows as a consequence of the uniqueness statement and the a priori bound obtained in the proof of Theorem 1.1. See Section 7.) Let $u, v \in C\left(\mathbb{R} ; H^{s}(\mathbb{T})\right)$ be two solutions to (1.5) with the same initial data with some smooth approximating solutions $\left\{u_{n}\right\}_{n \in \mathbb{N}}$ and $\left\{v_{m}\right\}_{m \in \mathbb{N}}$, respectively. Then, given $T>0$, we have

$$
\|u-v\|_{C_{T} H^{s}} \leqslant\left\|u-u_{n}\right\|_{C_{T} H^{s}}+\left\|u_{n}-v_{m}\right\|_{C_{T} H^{s}}+\left\|v_{m}-v\right\|_{C_{T} H^{s}},
$$

where $C_{T} H^{s}=C\left([-T, T] ; H^{s}(\mathbb{T})\right)$. The first and third terms on the right-hand side tend to 0 as $n, m \rightarrow \infty$. We, however, do not have any way to compare $u_{n}$ and $v_{m}$ in general, since we do not even know how these solutions $u$ and $v$ are constructed. Nonetheless, our enhanced uniqueness in Theorem 1.5 allows us to conclude that $u \equiv v$.

In establishing an energy estimate for the difference of two solutions $u$ and $v$ to (1.5) with the same initial condition, we perform an infinite iteration of normal form reductions (= integration by parts in time. In fact, this process basically corresponds to the Poincaré-Dulac normal form reductions. See the introduction in [18].) In [18], the first author with Guo and Kwon proved unconditional well-posedness of the cubic NLS on $\mathbb{T}$ in low regularity by performing normal form reductions infinitely many times. See also [11]. (On the one hand, we implemented an infinite iteration of normal form reductions in [11]. On the other hand, symmetrization at each step played a crucial role in [11]. In this paper, we will not employ such a symmetrization argument.) In our current setting, we do not work at the level of the equation (1.5) unlike [18]. We instead implement an infinite iteration scheme of normal form reductions for the evolution equations satisfied by energy quantities. See (1.16) and (1.18) below. 
We first apply an infinite iteration of normal form reductions to a solution $u \in$ $C\left([-T, T] ; H^{s}(\mathbb{T})\right)$ to $(1.5)$ and re-express $u$ as

$$
\begin{aligned}
& |\widehat{u}(n, t)|^{2}-\left|\widehat{u}_{0}(n)\right|^{2}=\mathfrak{S}_{\infty}(u)(n, t) \\
& \quad:=\left.\sum_{j=2}^{\infty} \mathcal{N}_{0}^{(j)}(u)\left(n, t^{\prime}\right)\right|_{0} ^{t}+\int_{0}^{t}\left[\sum_{j=2}^{\infty} \mathcal{R}^{(j)}(u)\left(n, t^{\prime}\right)+\sum_{j=1}^{\infty} \mathcal{N}_{1}^{(j)}(u)\left(n, t^{\prime}\right)\right] d t,
\end{aligned}
$$

where $\mathcal{N}_{0}^{(j)}$ (and $\mathcal{R}^{(j)}$ and $\left.\mathcal{N}_{1}^{(j)}\right)$ are $2 j$-linear forms $((2 j+2)$-linear forms, respectively). (More precisely, for fixed $t \in \mathbb{R},\left\{\mathcal{N}_{0}^{(j)}(n, t)\right\}_{n \in \mathbb{Z}}$ is a sequence of $2 j$-linear forms. Equivalently, by viewing $\mathcal{N}_{0}^{(j)}(u)(n, t)$ as the Fourier coefficient of $\mathcal{N}_{0}^{(j)}(u)(t)$, we can view $\mathcal{N}_{0}^{(j)}(\cdot)(t)$ as a $2 j$-linear operator. With abuse of terminology, however, we simply refer to $\mathcal{N}_{0}^{(j)}$ as a $2 j$-linear form in the following. A similar comment applies to $\mathcal{R}^{(j)}$ and $\mathcal{N}_{1}^{(j)}$.) Moreover, we show that these multilinear forms are bounded in $C\left([-T, T] ; H^{s}(\mathbb{T})\right), s>-\frac{1}{3}$, uniformly in $n \in \mathbb{Z}$. (In fact, we show that they are absolutely summable over $n \in \mathbb{Z}$.) See Proposition 7.1 below for a precise statement.

Now, take two solutions $u$ and $v$ to (1.5) constructed in Theorem 1.1 with the same initial condition $\left.u\right|_{t=0}=\left.v\right|_{t=0}=u_{0}$, satisfying $\|u\|_{C_{T} H^{s}},\|v\|_{C_{T} H^{s}} \lesssim\left\|u_{0}\right\|_{H^{s}}$. Then, with (1.5), we have

$$
\begin{aligned}
\frac{d}{d t}\|u(t)-v(t)\|_{H^{s}}^{2}= & -2 \operatorname{Re} i \sum_{n \in \mathbb{Z}}\langle n\rangle^{2 s}[\widehat{\mathcal{N}(u)}(n)-\widehat{\mathcal{N}(v)}(n)] \overline{(\widehat{u}(n)-\widehat{v}(n))} \\
& +2 \operatorname{Re} i \sum_{n \in \mathbb{Z}}\langle n\rangle^{2 s}[\widehat{\mathcal{R}(u)}(n)-\widehat{\mathcal{R}(v)}(n)] \overline{(\widehat{u}(n)-\widehat{v}(n))} \\
= & \mathrm{I}+\mathrm{II} .
\end{aligned}
$$

Let us only consider the second term II, corresponding to the resonant contribution. Using (1.14) with $\left.u\right|_{t=0}=\left.v\right|_{t=0}=u_{0}$ and (1.8), we obtain

$$
\begin{aligned}
|\mathrm{II}(t)| & \lesssim\left|\sum_{n \in \mathbb{Z}}\langle n\rangle^{2 s}\left(|\widehat{u}(n, t)|^{2}-|\widehat{v}(n, t)|^{2}\right) \overline{(\widehat{u}(n)-\widehat{v}(n))} \widehat{v}(n)\right| \\
& \leqslant \sup _{m \in \mathbb{Z}}\left|\mathfrak{S}_{\infty}(u)(m, t)-\mathfrak{S}_{\infty}(v)(m, t)\right| \cdot \sum_{n \in \mathbb{Z}}\langle n\rangle^{2 s}|\widehat{u}(n)-\widehat{v}(n)||\widehat{v}(n)| \\
& \leqslant C\left(\left\|u_{0}\right\|_{H^{s}}\right)\|u-v\|_{C_{T} H^{s}}^{2},
\end{aligned}
$$

where we used the multilinearity of $\mathcal{N}_{0}^{(j)}, \mathcal{R}^{(j)}$, and $\mathcal{N}_{1}^{(j)}$ along with their $C\left([-T, T] H^{s}(\mathbb{T})\right)$-bounds to control the first factor and Cauchy-Schwarz 
inequality on the second factor. As for the nonresonant contribution I in (1.15), we expand it into a sum of infinite series analogous to (1.14) and obtain an estimate of the form:

$$
|\mathrm{I}(t)| \leqslant C\left(\left\|u_{0}\right\|_{H^{s}}\right)\|u-v\|_{C_{T} H^{s}}^{2} .
$$

See Proposition 7.2 and Section 8.6. This yields the desired energy estimate for the difference of two solutions with the same initial condition.

Therefore, the main task is to prove the identity (1.14) with good estimates. We achieve this goal by performing integration by parts in an iterative manner, which introduces nonlinear terms of higher and higher degrees. While these nonlinear terms thus introduced are of higher degrees, it turns out that they satisfy better estimates. Namely, this infinite iteration of normal form reductions allows us to exchange analytical difficulty with combinatorial and notational complexity. In order to keep track of all possible ways to perform integration by parts, we introduce the notion of ordered bi-trees. We devote Section 8 for presenting the normal form reductions.

Finally, we point out the connection to the $I$-method. (The connection between normal form reductions and modified energies in the $I$-method has already been pointed out in [19].) At each step of integration by parts, we introduce boundary terms. This corresponds to adding a correction term appearing in the $I$-method. Namely, in the context of the $I$-method, our approach is nothing but to compute and estimate a modified energy of an infinite order. (The highest order of modified energies used in the literature is three in the application of the $I$-method to the $\mathrm{KdV}$ equation [13], corresponding to two iterations of normal form reductions.) For example, our argument yields the following infinite expansion of the $H^{s}$ energy for a single solution:

$$
\begin{aligned}
\|u(t)\|_{H^{s}}^{2}-\|u(0)\|_{H^{s}}^{2}= & \left.\sum_{j=2}^{\infty} \sum_{n \in \mathbb{Z}}\langle n\rangle^{2 s} \mathcal{N}_{0}^{(j)}(u)\left(n, t^{\prime}\right)\right|_{0} ^{t} \\
& +\int_{0}^{t}\left[\sum_{j=2}^{\infty} \sum_{n \in \mathbb{Z}}\langle n\rangle^{2 s} \mathcal{R}^{(j)}(u)\left(n, t^{\prime}\right)\right. \\
& \left.+\sum_{j=1}^{\infty} \sum_{n \in \mathbb{Z}}\langle n\rangle^{2 s} \mathcal{N}_{1}^{(j)}(u)\left(n, t^{\prime}\right)\right] d t^{\prime} .
\end{aligned}
$$

See (8.53) below. Namely, defining a modified energy $E_{\infty}(u)$ of an infinite order by

$$
E_{\infty}(u)=\|u\|_{H^{s}}^{2}-\sum_{j=2}^{\infty} \sum_{n \in \mathbb{Z}}\langle n\rangle^{2 s} \mathcal{N}_{0}^{(j)}(u)(n),
$$


we obtain

$$
\begin{aligned}
& E_{\infty}(u)(t)-E_{\infty}(u)(0) \\
& =\int_{0}^{t}\left[\sum_{j=2}^{\infty} \sum_{n \in \mathbb{Z}}\langle n\rangle^{2 s} \mathcal{R}^{(j)}(u)\left(n, t^{\prime}\right)+\sum_{j=1}^{\infty} \sum_{n \in \mathbb{Z}}\langle n\rangle^{2 s} \mathcal{N}_{1}^{(j)}(u)\left(n, t^{\prime}\right)\right] d t^{\prime} .
\end{aligned}
$$

While we do not need the modified energy $E_{\infty}(u)$ in this paper, such an expansion by adding an infinite sequence of correction terms seems to be new and of interest.

As for the difference of two solutions with the same initial data, while there are contributions from the resonant part as well as the cross terms ( $\mathrm{I}_{u v}$ and $\mathrm{I}_{v u}$ below) in the nonresonant part, we also have a similar infinite expansion (with two factors of $u-v)$ :

$$
\begin{aligned}
\| u(t)- & v(t) \|_{H^{s}}^{2} \\
= & \int_{0}^{t}\left\{\left(\mathrm{I}_{u u}\left(t^{\prime}\right)-\mathrm{I}_{u v}\left(t^{\prime}\right)\right)-\left(\mathrm{I}_{v u}\left(t^{\prime}\right)-\mathrm{I}_{v v}\left(t^{\prime}\right)\right)\right\} d t^{\prime} \\
& +\int_{0}^{t} \sum_{n \in \mathbb{Z}}\langle n\rangle^{2 s}\left(\mathfrak{S}_{\infty}(u)\left(n, t^{\prime}\right)-\mathfrak{S}_{\infty}(v)\left(n, t^{\prime}\right)\right) \overline{\left(\widehat{u}_{n}-\widehat{v}_{n}\right)} \widehat{v}_{n}\left(t^{\prime}\right) d t^{\prime},
\end{aligned}
$$

where the second term on the right-hand side involves a sum of infinite series in view of (1.14). As for the integrands in the first integral, see (8.51), (8.53), (8.54), (8.58), and (8.59), where each integrand is written as a sum of infinite series. See Remark 8.20.

We conclude this introduction by stressing that reducing the $H^{s}$-energy functionals to the infinite series expansions (1.16) and (1.18) (also see (1.17)) is the main novelty of this paper. In the proof of Theorem 1.5, we use the infinite series expansion (1.18) for the difference of two solutions with the same initial data to prove uniqueness of solutions to (1.5). In [33], (a variant of) the infinite series expansion (1.16) for a single solution plays an important role in establishing a crucial energy estimate in studying the transport property of Gaussian measures on periodic functions under the flow of 4NLS (1.1). See Remark 1.6. We hope that this idea of expanding energy functionals into infinite series by normal form reductions can be applied to other equations in various settings.

REMARK 1.6. In a recent work [33], the first author with Sosoe and Tzvetkov established an optimal regularity result for quasi-invariance of the Gaussian measures on Sobolev spaces under the original 4NLS (1.1) by implementing a similar infinite iteration of normal form reductions on the $H^{s}$-functional for solutions to (1.1) for $s \in\left(\frac{1}{2}, 1\right)$. While there are similarities between the normal form approach in [33] and in Section 8 of this paper, more care is required 
in the present paper since we need to gain derivatives at each step of normal form reductions in order to estimate the multilinear forms $\mathcal{N}_{0}^{(j)}(u), \mathcal{R}^{(j)}(u)$, and $\mathcal{N}_{1}^{(j)}(u)$ of arbitrarily large degrees in terms of the negative Sobolev norm of $u$.

REMARK 1.7. Recall that the mean-zero Gaussian white noise on $\mathbb{T}$ is formally given by

$$
d \mu=Z^{-1} e^{-1 / 2\|\phi\|_{L^{2}}^{2}} d \phi .
$$

In particular, a typical element under $\mu$ is given by

$$
\phi^{\omega}(x)=\phi(x ; \omega)=\sum_{n \in \mathbb{Z}} g_{n}(\omega) e^{i n x},
$$

where $\left\{g_{n}\right\}_{n \in \mathbb{Z}}$ is a sequence of independent standard complex-valued Gaussian random variables on a probability space $(\Omega, \mathcal{F}, P)$. (In (1.19), we dropped the harmless factor of $2 \pi$. The same comment applies to the remaining part of this paper.) From (1.19), it is easy to see that $\phi^{\omega}$ in (1.19) lies in $H^{s}(\mathbb{T}) \backslash H^{-1 / 2}(\mathbb{T})$, $s<-\frac{1}{2}$, almost surely. In particular, the regularity of the white noise is below the regularities stated in Theorems 1.1 and 1.5.

In view of the $L^{2}$-conservation for (1.5) and the Hamiltonian structure of the equation, one may expect that the white noise is invariant under the dynamics of (1.5). In [37], the authors with Tzvetkov proved that this is indeed the case. The main difficulty in [37] lies in constructing local-in-time dynamics with respect to the random initial data (1.19), which was overcome by a combination of new stochastic analysis and deterministic analysis different from the analysis presented in this paper.

REMARK 1.8. We can also start our discussion with the more general cubic fourth order NLS (1.2) with $\mu \neq 0$ and consider its renormalized version. In this case, the following phase function

$$
\begin{aligned}
\phi_{\lambda, \mu}(\bar{n}) & =-\lambda\left(n_{1}^{2}-n_{2}^{2}+n_{3}^{2}-n^{2}\right)+\mu\left(n_{1}^{4}-n_{2}^{4}+n_{3}^{4}-n^{4}\right) \\
& =\left(n_{1}-n_{2}\right)\left(n_{1}-n\right)\left\{-2 \lambda+\mu\left(n_{1}^{2}+n_{2}^{2}+n_{3}^{2}+n^{2}+2\left(n_{1}+n_{3}\right)^{2}\right)\right\}
\end{aligned}
$$

plays an important role in the analysis. Compare this with (2.4).

If the last factor in (1.20) does not vanish for any $n_{1}, n_{2}, n_{3}, n \in \mathbb{Z}$, then the main results in this paper clearly hold with the same proofs. Note that even if the last factor may be 0 , that is, $2 \lambda \in \mu \mathbb{N}$, the new resonance occurs only for low frequencies, where $\max \left(n_{1}^{2}, n_{2}^{2}, n_{3}^{2}, n^{2}\right) \lesssim \lambda / \mu$, and hence the same argument basically holds. 
This paper is organized as follows. In Section 2, we introduce notations and the function spaces along with their basic properties. In Section 3, we present multilinear Strichartz estimates, which are then used to prove the crucial trilinear estimate (1.10) and the energy estimate (1.11) in Sections 4 and 5, respectively. In Section 6, we present the proof of global existence (Theorem 1.1). In particular, given any $T>0$, we choose $M=M(T) \gg 1$ such that the estimates (1.9)(1.11) adapted to the parameter $M$ allow us to construct solutions on the time interval $[-T, T]$. In Section 7, by assuming the key propositions (Propositions 7.1 and 7.2), we prove uniqueness of solutions to (1.5) in $H^{s}(\mathbb{T})$ for $s>-\frac{1}{3}$, which is then used to establish continuous dependence on initial data and thus global wellposedness (Theorem 1.5). In Section 8, we present details of the normal form reductions and prove Propositions 7.1 and 7.2.

\section{Notations, function spaces, and their basic properties}

2.1. Notations. For $a, b>0$, we use $a \lesssim b$ to mean that there exists $C>0$ such that $a \leqslant C b$. By $a \sim b$, we mean that $a \lesssim b$ and $b \lesssim a$. We also use $a+$ (and $a-$ ) to denote $a+\varepsilon$ (and $a-\varepsilon$, respectively) for arbitrarily small $\varepsilon \ll 1$.

Given a function $u$ on $\mathbb{T} \times \mathbb{R}$, we use $\widehat{u}$ and $\mathcal{F}(u)$ to denote the space-time Fourier transform of $u$ given by

$$
\widehat{u}(n, \tau)=\int_{\mathbb{T} \times \mathbb{R}} e^{-i n x} e^{-i t \tau} u(x, t) d x d t .
$$

When there is no confusion, we may simply use $\widehat{u}$ or $\mathcal{F}(u)$ to denote the spatial, temporal, or space-time Fourier transform of $u$, depending on the context. In dealing with the spatial Fourier transform, we often denote $\widehat{u}(n, t)$ by $\widehat{u}_{n}(t)$.

For $k \in \mathbb{Z}_{\geqslant 0}:=\mathbb{Z} \cap[0, \infty)$, we define the dyadic intervals $I_{k}$ by setting $I_{0}=$ $\{\xi:|\xi|<1\}$ and $I_{k}=\left\{\xi: 2^{k-1} \leqslant|\xi|<2^{k}\right\}$ for $k \geqslant 1$. Next, we define the dyadic intervals $I_{k}^{M}, k \geqslant \log _{2} M$, adapted to a given dyadic parameter $M \geqslant 1$, by setting

$$
I_{k}^{M}= \begin{cases}I_{k}, & k>\log _{2} M, \\ \bigcup_{k=0}^{\log _{2} M} I_{k}, & k=\log _{2} M .\end{cases}
$$

For simplicity, when $k=\log _{2} M$, we set

$$
I_{\mathrm{low}}^{M}=I_{\log _{2} M}^{M} .
$$

In the following, all the definitions depend on this dyadic parameter $M \geqslant 1$. For convenience, we set

$$
\mathbb{Z}_{M}=\mathbb{Z} \cap\left[\log _{2} M, \infty\right)
$$


For $k \in \mathbb{Z}_{M}$ and $j \geqslant 0$, let

$$
D_{k, j}^{M}=\left\{(n, \tau) \in \mathbb{Z} \times \mathbb{R}: n \in I_{k}^{M}, \tau+n^{4} \in I_{j}\right\}
$$

and $D_{k, \leqslant j}^{M}=\bigcup_{j^{\prime} \leqslant j} D_{k, j^{\prime}}^{M}$. We also define $D_{\leqslant j}$ by $D_{\leqslant j}=\bigcup_{k \in \mathbb{Z}_{M}} D_{k, \leqslant j}^{M}$.

For $k \in \mathbb{Z}_{M}$, we use $\mathbf{P}_{k}$ to denote the projection operator on $L^{2}(\mathbb{T})$ defined by $\widehat{\mathbf{P}_{k} u}(n)=\mathbf{1}_{I_{k}^{M}}(n) \widehat{u}(n)$. Note that $\mathbf{P}_{\log _{2} M}$ is the projection onto 'low' frequencies $\{|n| \lesssim M\}$. With a slight abuse of notation, we also use $\mathbf{P}_{k}$ to denote the projection operator on $L^{2}(\mathbb{T} \times \mathbb{R})$ given by $\mathcal{F}\left(\mathbf{P}_{k} u\right)(n, \tau)=\mathbf{1}_{I_{k}^{M}}(n) \mathcal{F}(u)(n, \tau)$. We also set

$$
\mathbf{P}_{\leqslant k}=\sum_{\log _{2}} \mathbf{P}_{\ell \leqslant \ell \leqslant k} \quad \text { and } \quad \mathbf{P}_{>k}=\sum_{\ell>k} \mathbf{P}_{\ell}
$$

Let $\eta_{0}: \mathbb{R} \rightarrow[0,1]$ be an even smooth cutoff function supported on $\left[-\frac{8}{5}, \frac{8}{5}\right]$ such that $\eta_{0} \equiv 1$ on $\left[-\frac{5}{4}, \frac{5}{4}\right]$. We define $\eta$ by $\eta(\xi)=\eta_{0}(\xi)-\eta_{0}(2 \xi)$, and set $\eta_{k}(\xi)=\eta\left(2^{-k} \xi\right)$ for $k \in \mathbb{Z}$. Namely, $\eta_{k}$ is supported on $\left\{\frac{5}{4} \cdot 2^{k-1} \leqslant|\xi| \leqslant \frac{8}{5} \cdot 2^{k}\right\}$. As before, we define $\eta_{\leqslant k}=\sum_{\ell \leqslant k} \eta_{\ell}$, and so on.

Given a set of indices such as $j_{i}$ and $k_{i}, i=1, \ldots, 4$, we use $j_{i}^{*}$ and $k_{i}^{*}$ to denote the decreasing rearrangements of these indices. Also, given a set of frequencies $n_{i}, i=1, \ldots, 4$, we use $n_{i}^{*}$ to denote the decreasing rearrangements of $\left|n_{i}\right|, i=1$, $\ldots, 4$.

In the following, we use $S(t)=e^{-i t \partial_{x}^{4}}$ to denote the solution operator to the linear fourth order Schrödinger equation: $i \partial_{t} u=\partial_{x}^{4} u$. Namely, for $f \in L^{2}(\mathbb{T})$, we have

$$
S(t) f=\sum_{n \in \mathbb{Z}} e^{i n x-i n^{4} t} \widehat{f}(n) .
$$

In performing normal form reductions in Section 8, we use the following interaction representation $\mathbf{u}($ of $u)$ on $\mathbb{T} \times \mathbb{R}$ :

$$
\mathbf{u}(t):=S(-t) u(t)=e^{i t \partial_{x}^{4}} u(t) .
$$

On the Fourier side, we have $\widehat{\mathbf{u}}_{n}(t)=e^{i t n^{4}} \widehat{u}_{n}(t), n \in \mathbb{Z}$. With this notation, (1.5) can be written as

$$
\begin{aligned}
\partial_{t} \widehat{\mathbf{u}}_{n} & =-i \sum_{\Gamma(n)} e^{-i \phi(\bar{n}) t} \widehat{\mathbf{u}}_{n_{1}} \widehat{\mathbf{u}}_{n_{2}} \widehat{\mathbf{u}}_{n_{3}}+i\left|\widehat{\mathbf{u}}_{n}\right|^{2} \widehat{\mathbf{u}}_{n} \\
& =:-i \mathrm{~N}(\mathbf{u})_{n}(t)+i \mathrm{R}(\mathbf{u})_{n}(t),
\end{aligned}
$$

where the plane $\Gamma(n)$ is defined

$$
\Gamma(n)=\left\{\left(n_{1}, n_{2}, n_{3}\right) \in \mathbb{Z}^{3}: n=n_{1}-n_{2}+n_{3} \text { and } n_{1}, n_{3} \neq n\right\}
$$


and the phase function $\phi(\bar{n})$ is defined by

$$
\begin{aligned}
\phi(\bar{n}) & =\phi\left(n_{1}, n_{2}, n_{3}, n\right)=n_{1}^{4}-n_{2}^{4}+n_{3}^{4}-n^{4} \\
& =-\left(n-n_{1}\right)\left(n-n_{3}\right)\left(n_{1}^{2}+n_{2}^{2}+n_{3}^{2}+n^{2}+2\left(n_{1}+n_{3}\right)^{2}\right) .
\end{aligned}
$$

Here, the last equality holds under $n=n_{1}-n_{2}+n_{3}$. See [36, Lemma 3.1].

We also recall the phase function $\mu(\bar{n})$ for the usual Schrödinger equation:

$$
\begin{aligned}
\mu(\bar{n}) & :=-n_{1}^{2}+n_{2}^{2}-n_{3}^{2}+n^{2} \\
& =2\left(n_{2}-n_{1}\right)\left(n_{2}-n_{3}\right)=2\left(n-n_{1}\right)\left(n-n_{3}\right),
\end{aligned}
$$

where the last two equalities hold under $n=n_{1}-n_{2}+n_{3}$.

2.2. Function spaces and their basic properties. Recall the definition of the standard Sobolev space $H^{s}(\mathbb{T})$ :

$$
\|f\|_{H^{s}}=\left\|\langle n\rangle^{s} \widehat{f}(n)\right\|_{\ell_{n}^{2}},
$$

where $\langle\cdot\rangle=\left(1+|\cdot|^{2}\right)^{1 / 2}$. Given $M \geqslant 1$, we define the $H_{M}^{s}$-norm adapted to the parameter $M \geqslant 1$ by

$$
\|f\|_{H_{M}^{s}}=\left\|\left(M^{2}+n^{2}\right)^{s / 2} \widehat{f}(n)\right\|_{\ell_{n}^{2}} .
$$

Clearly, the $H_{M}^{s}$-norm is equivalent to the standard $H^{s}$-norm. When $s<0$, however, it follows from the dominated convergence theorem that

$$
\lim _{M \rightarrow \infty}\|f\|_{H_{M}^{s}}=0
$$

for all $f \in H^{s}(\mathbb{T})$. This decay property (2.6) plays an important role in our analysis.

Next, we define our solution space adapted to this parameter $M \geqslant 1$. In [3], Bourgain introduced the dispersive Sobolev space $X^{s, b}(\mathbb{T} \times \mathbb{R})$ via the norm:

$$
\|u\|_{X^{s, b}(\mathbb{T} \times \mathbb{R})}=\left\|\langle n\rangle^{s}\left\langle\tau+n^{4}\right\rangle^{b} \widehat{u}(n, \tau)\right\|_{\ell_{n}^{2} L_{\tau}^{2}(\mathbb{Z} \times \mathbb{R})}=\left\|\left\langle\partial_{x}\right\rangle^{s}\left\langle\partial_{t}\right\rangle^{b} \mathbf{u}\right\|_{L^{2}(\mathbb{T} \times \mathbb{R})},
$$

where $\mathbf{u}$ is the interaction representation defined in (2.1). The Fourier restriction norm method, utilizing the $X^{s, b}$-spaces and their variants, has been very effective in studying nonlinear evolution equations in low regularity settings. In the following, we consider the $X^{s, b}$-spaces adapted to short-time scales and the parameter $M \geqslant 1$. When $M=1$, these spaces were introduced by Ionescu et al. [21] in the context of the KP-I equation. Also, see Christ et al. [10] and Koch and Tataru [26, 27] for similar definitions. While we state some basic 
properties of these function spaces, we refer readers to [19] for the details of their proofs.

Fix $M \geqslant 1$. For $k \in \mathbb{Z}_{M}$, we define the dyadic $X^{s, b}$-type spaces $X_{M, k}$ by

$$
\begin{aligned}
& X_{M, k}=\left\{f_{k} \in L^{2}(\mathbb{Z} \times \mathbb{R}): f_{k}(n, \tau) \text { is supported on } I_{k}^{M} \times \mathbb{R}\right. \\
& \left.\quad \text { and }\left\|f_{k}\right\|_{X_{M, k}}:=\sum_{j=0}^{\infty} 2^{j / 2}\left\|\eta_{j}\left(\tau+n^{4}\right) f_{k}(n, \tau)\right\|_{\ell_{n}^{2} L_{\tau}^{2}}<\infty\right\} .
\end{aligned}
$$

Then, the following properties hold for $X_{M, k}, k \in \mathbb{Z}_{M}$ (with implicit constants independent of $M \geqslant 1$ ):

(i) We have

$$
\left\|\int_{\mathbb{R}}\left|f_{k}(n, \tau)\right| d \tau\right\|_{\ell_{n}^{2}} \lesssim\left\|f_{k}\right\|_{X_{M, k}} \quad \text { and } \quad \int_{\mathbb{R}}\left\|g_{k}(n, \tau)\right\|_{\ell_{n}^{2}} d \tau \lesssim\left\|f_{k}\right\|_{X_{M, k}}
$$

for all $f_{k} \in X_{M, k}$, where $g_{k}(n, \tau)=f_{k}\left(n, \tau-n^{4}\right)$.

(ii) For $k, \ell \in \mathbb{Z}_{M}$ and $f_{k} \in X_{k}$, we have then

$$
\begin{aligned}
& \sum_{j=\ell+1}^{\infty} 2^{j / 2}\left\|\eta_{j}\left(\tau+n^{4}\right) \int_{\mathbb{R}}\left|f_{k}\left(n, \tau^{\prime}\right)\right| 2^{-\ell}\left(1+2^{-\ell}\left|\tau-\tau^{\prime}\right|\right)^{-4} d \tau^{\prime}\right\|_{\ell_{n}^{2} L_{\tau}^{2}} \\
& \quad+2^{\ell / 2}\left\|\eta_{\leqslant \ell}\left(\tau+n^{4}\right) \int_{\mathbb{R}}\left|f_{k}\left(n, \tau^{\prime}\right)\right| 2^{-\ell}\left(1+2^{-\ell}\left|\tau-\tau^{\prime}\right|\right)^{-4} d \tau^{\prime}\right\|_{\ell_{n}^{2} L_{\tau}^{2}} \\
& \lesssim\left\|f_{k}\right\|_{X_{M, k}},
\end{aligned}
$$

where the implicit constant is independent of $k$ and $\ell$. See [17] for the proof.

(iii) As a consequence of (ii), we have

$$
\left\|\mathcal{F}\left[\gamma\left(2^{\ell}\left(t-t_{0}\right)\right) \cdot \mathcal{F}^{-1}\left(f_{k}\right)\right]\right\|_{X_{M, k}} \lesssim\left\|f_{k}\right\|_{X_{M, k}}
$$

for $k, \ell \in \mathbb{Z}_{M}, t_{0} \in \mathbb{R}, f_{k} \in X_{M, k}$ and $\gamma \in \mathcal{S}(\mathbb{R})$, where the implicit constant in (2.9) is also independent of $k, \ell$, and $t_{0}$.

Next, we consider the time localization of the $X_{M, k}$-space onto the time scale $\sim 2^{-[\alpha k]}$, where $\alpha>0$ is to be determined later. Here, $[x]$ denotes the integer part of $x$. For $k \in \mathbb{Z}_{M}$ we define the spaces $F_{M, k}^{\alpha}$ and $N_{M, k}^{\alpha}$ by

$$
\begin{aligned}
F_{M, k}^{\alpha}=\left\{u \in L^{2}(\mathbb{T} \times \mathbb{R}): \widehat{u}(n, \tau) \text { is supported in } I_{k}^{M} \times \mathbb{R}\right. \\
\left.\quad \text { and }\|u\|_{F_{M, k}^{\alpha}}=\sup _{t_{k} \in \mathbb{R}}\left\|\mathcal{F}\left[\eta_{0}\left(2^{[\alpha k]}\left(t-t_{k}\right)\right) \cdot u\right]\right\|_{X_{M, k}}<\infty\right\},
\end{aligned}
$$


$N_{M, k}^{\alpha}=\left\{u \in L^{2}(\mathbb{T} \times \mathbb{R}): \widehat{u}(n, \tau)\right.$ is supported in $I_{k}^{M} \times \mathbb{R}$ and

$$
\left.\|u\|_{N_{M, k}^{\alpha}}=\sup _{t_{k} \in \mathbb{R}}\left\|\left(\tau+n^{4}+i 2^{[\alpha k]}\right)^{-1} \mathcal{F}\left[\eta_{0}\left(2^{[\alpha k]}\left(t-t_{k}\right)\right) \cdot u\right]\right\|_{X_{M, k}}<\infty\right\} .
$$

Given $T>0$, we define the time restriction spaces $F_{M, k}^{\alpha}(T)$ and $N_{M, k}^{\alpha}(T)$ by

$$
\begin{aligned}
F_{M, k}^{\alpha}(T) & =\left\{u \in C\left([-T, T] ; L^{2}(\mathbb{T})\right):\|u\|_{F_{M, k}^{\alpha}(T)}=\inf _{\widetilde{u}=u \text { on } \mathbb{T} \times[-T, T]}\|\widetilde{u}\|_{F_{M, k}^{\alpha}}\right\}, \\
N_{M, k}^{\alpha}(T) & =\left\{u \in C\left([-T, T] ; L^{2}(\mathbb{T})\right):\|u\|_{N_{M, k}^{\alpha}(T)}=\inf _{\widetilde{u}=u \text { on } \mathbb{T} \times[-T, T]}\|\widetilde{u}\|_{N_{M, k}^{\alpha}}\right\} .
\end{aligned}
$$

Here, the infimum is taken over all extensions $\widetilde{u} \in C_{0}\left(\mathbb{R} ; L^{2}(\mathbb{T})\right)$.

We finally define our solution space $F_{M}^{s, \alpha}(T)$ and its dual space $N_{M}^{s, \alpha}(T)$ by putting together the dyadic spaces defined above via the Littlewood-Paley decomposition. For $s \in \mathbb{R}, \alpha>0$, and $T>0$, we define the spaces $F_{M}^{s, \alpha}(T)$ and $N_{M}^{s, \alpha}(T)$ by

$$
\begin{aligned}
& F_{M}^{s, \alpha}(T)=\left\{u:\|u\|_{F_{M}^{s}(T)}^{2}=\sum_{k \in \mathbb{Z}_{M}} 2^{2 k s}\left\|\mathbf{P}_{k} u\right\|_{F_{M, k}^{\alpha}(T)}^{2}<\infty\right\}, \\
& N_{M}^{s, \alpha}(T)=\left\{u:\|u\|_{N_{M}^{s}(T)}^{2}=\sum_{k \in \mathbb{Z}_{M}} 2^{2 k s}\left\|\mathbf{P}_{k} u\right\|_{N_{M, k}^{\alpha}(T)}^{2}<\infty\right\} .
\end{aligned}
$$

Here, $\alpha=\alpha(s)>0$ is a parameter to be chosen later. See Section 2.4. When $M=1$, we simply drop the subscript $M$ from the function spaces and use $F^{s, \alpha}(T)$, and so on.

In order to handle the short-time structure embedded in the definitions of $F_{M}^{s, \alpha}(T)$ and $N_{M}^{s, \alpha}(T)$, we define the corresponding energy space $E_{M}^{s}(T)$ by

$$
\|u\|_{E_{M}^{s}(T)}^{2}=\sum_{k \in \mathbb{Z}_{M}} \sup _{t_{k} \in[-T, T]} 2^{2 k s}\left\|\mathbf{P}_{k} u\left(t_{k}\right)\right\|_{L^{2}(\mathbb{T})}^{2}
$$

for $u \in C\left([-T, T] ; H^{\infty}(\mathbb{T})\right)$. While the definition of $E_{M}^{s}(T)$ depends on $M \geqslant 1$, it is independent of the parameter $\alpha>0$. This space is essentially the usual energy space $C\left([-T, T] ; H_{M}^{s}(\mathbb{T})\right)$ but with a logarithmic difference. See Section 2.3.

We conclude this subsection by recalling some basic lemmas from [19]. While these properties are stated and proved for $M=1$ in [19], a straightforward modification yields the corresponding statements for $M \geqslant 1$ (with implicit constants independent of $M \geqslant 1$ ). In the following, we fix $M \geqslant 1$ and $\alpha>0$.

The first lemma shows that a smooth time cutoff supported on an interval of size $\sim 2^{-[\alpha k]}$ acts boundedly on $N_{M, k}^{\alpha}$. 
LEMMA 2.1. Let $k \in \mathbb{Z}_{M}, t_{k} \in \mathbb{R}$, and $\gamma \in \mathcal{S}(\mathbb{R})$. Then, we have

$$
\begin{gathered}
\left\|\left(\tau+n^{4}+i 2^{[\alpha k]}\right)^{-1} \mathcal{F}\left[\gamma\left(2^{[\alpha k]}\left(t-t_{k}\right)\right) \cdot \mathcal{F}^{-1}\left(f_{k}\right)\right]\right\|_{X_{M, k}} \\
\lesssim\left\|\left(\tau+n^{4}+i 2^{[\alpha k]}\right)^{-1} f_{k}\right\|_{X_{M, k}}
\end{gathered}
$$

for $f_{k}$ supported on $I_{k}^{M} \times \mathbb{R}$. Here, the implicit constant is independent of $M, \alpha$, $k$, and $t_{k}$.

The second lemma shows that $F_{k}^{\alpha}$ - and $F^{s, \alpha}$-norms control the supremum in time (of the appropriate spatial norms).

LEMMA 2.2. (i) Let $u$ be a function on $\mathbb{T} \times \mathbb{R}$ such that supp $\widehat{u} \subset I_{k}^{M} \times \mathbb{R}$, $k \in \mathbb{Z}_{M}$. Then, we have

$$
\|u\|_{L_{t}^{\infty} L_{x}^{2}} \lesssim\|u\|_{F_{M, k}^{\alpha}}
$$

Similarly, we have

$$
\left\|\mathcal{F}^{-1}\left[\eta_{\leqslant j}\left(\tau+n^{4}\right) \widehat{u}\right]\right\|_{L_{t}^{\infty} L_{x}^{2}} \lesssim\|u\|_{F_{M, k}^{\alpha}}
$$

for any $j \in \mathbb{Z}_{\geqslant 0}$. Here, (2.11) also holds when we replace $\eta_{\leqslant j}$ by $\eta_{j}$ or $\eta_{>j}$.

(ii) Let $s \in \mathbb{R}$ and $T>0$. Then, we have

$$
\sup _{t \in[-T, T]}\|u(t)\|_{H_{M}^{s}} \lesssim\|u\|_{F_{M}^{s, \alpha}(T)} .
$$

In the following, we define the corresponding function spaces with the temporal regularity $b$. For $k \in \mathbb{Z}_{M}$ and $b \in \mathbb{R}$, define $X_{M, k}^{b}$ by

$$
\left\|f_{k}\right\|_{X_{M, k}^{b}}:=\sum_{j=0}^{\infty} 2^{j b}\left\|\eta_{j}\left(\tau+n^{4}\right) f_{k}(n, \tau)\right\|_{\ell_{n}^{2} L_{\tau}^{2}}
$$

for $f_{k}$ supported on $I_{k}^{M} \times \mathbb{R}$. Note that $X_{M, k}=X_{M, k}^{1 / 2}$. Then, we define the spaces $F_{M, k}^{b, \alpha}$ and $F_{M}^{s, b, \alpha}(T)$ with $X_{M, k}^{b}$, just as we defined $F_{M, k}^{\alpha}$ and $F^{s, \alpha}(T)$ with $X_{M, k}$.

The following lemma allows us to gain a small power of time localization at a slight expense of the regularity in modulation.

LeMmA 2.3. Let $T>0$ and $b<\frac{1}{2}$. Then, we have

$$
\left\|\mathbf{P}_{k} u\right\|_{F_{M, k}^{b, \alpha}} \lesssim T^{1 / 2-b-}\left\|\mathbf{P}_{k} u\right\|_{F_{M, k}^{\alpha}}
$$

for any function u supported on $\mathbb{T} \times[-T, T]$. 
The following lemma shows that the multiplication by a sharp cutoff function in time is 'almost' bounded in $X_{M, k}$.

LEMMA 2.4. Let $k \in \mathbb{Z}_{M}$. Then, for any interval $I=\left[t_{1}, t_{2}\right] \subset \mathbb{R}$, we have

$$
\sup _{j \in \mathbb{Z}_{\geqslant 0}} 2^{j / 2}\left\|\eta_{j}\left(\tau+n^{4}\right) \mathcal{F}\left[\mathbf{1}_{I}(t) \cdot \mathbf{P}_{k} u\right]\right\|_{\ell_{n}^{2} L_{\tau}^{2}} \lesssim\left\|\mathcal{F}\left(\mathbf{P}_{k} u\right)\right\|_{X_{M, k}},
$$

where the implicit constant is independent of $M, k$ and $I$.

Finally, we state a linear estimate associated with the fourth order Schrödinger equation.

LEMMA 2.5. Let $T>0$. Suppose that $u \in C\left([-T, T] ; H^{\infty}(\mathbb{T})\right)$ is a solution to the following nonhomogeneous linear fourth order Schrödinger equation:

$$
i \partial_{t} u-\partial_{x}^{4} u=v \quad \text { on } \mathbb{T} \times(-T, T),
$$

where $v \in C\left([-T, T] ; H^{\infty}(\mathbb{T})\right)$. Then, for any $s \in \mathbb{R}$ and $\alpha \geqslant 0$, we have

$$
\|u\|_{F_{M}^{s, \alpha}(T)} \lesssim\|u\|_{E_{M}^{s}(T)}+\|v\|_{N_{M}^{s, \alpha}(T)} .
$$

2.3. On the energy space. As pointed out above, the energy space $E_{M}^{s}(T)$ defined in (2.10) is essentially the usual energy space $C\left([-T, T] ; H_{M}^{s}(\mathbb{T})\right)$ but there is a logarithmic difference that we need to handle. In this subsection, we introduce a sequence $\left\{a_{k_{0}}\right\}_{k_{0} \in \mathbb{Z}_{M}}$ of symbols that allows us to control the $E_{M}^{s}$-norm. Similar symbols have been used in [26, 27].

Fix $k_{0} \in \mathbb{Z}_{M}$. For sufficiently small $\delta_{0}=\delta_{0}(s)>0$ (to be chosen later. See Proposition 5.2 below), we define a symbol $a_{k_{0}}^{0}$ on $\mathbb{R}$ by setting

$$
a_{k_{0}}^{0}(\xi)=|\xi|^{2 s} \min \left\{\frac{|\xi|}{2^{k_{0}}}, \frac{2^{k_{0}}}{|\xi|}\right\}^{\delta_{0}}
$$

for $|\xi|=2^{k}$ with $k \in \mathbb{Z}_{M}$ and we extend the definition of $a_{k_{0}}^{0}$ onto $\mathbb{R}$ by linear interpolation. As for the choice of $\delta_{0}>0$, see Proposition 5.2 below. In particular, it is constant on $[-M, M]$. As it is, $a_{k_{0}}^{0}$ is not smooth and thus we need to smooth it out.

Let $\eta_{0}: \mathbb{R} \rightarrow[0,1]$ be a smooth cutoff function with $\eta_{0}(\xi) \equiv 1$ for $|\xi| \leqslant \frac{5}{4}$ and $=0$ for $|\xi| \geqslant \frac{8}{5}$ as above. Then, choose $c_{0}>0$ such that $\int c_{0} \eta_{0}(\xi) d \xi=1$. Given $k \in \mathbb{Z}_{M}$, we define a symbol $a_{k_{0}}$ in a neighbourhood of a dyadic point $2^{k}$ by

$$
a_{k_{0}}(\xi)=\left(a_{k_{0}}^{0} * \theta_{k}\right)(\xi) \quad \text { on } J_{k}:=\left\{\xi \in \mathbb{R}:\left|\xi-2^{k}\right| \leqslant \frac{1}{4} \cdot 2^{k}\right\},
$$

where $\theta_{k}(\xi)=\left(10 c_{0} / 2^{k}\right) \eta_{0}\left(\left(10 / 2^{k}\right) \xi\right)$. For $\xi \notin \bigcup_{k \in \mathbb{Z}_{M}} J_{k}$, we set $a_{k_{0}}(\xi)=a_{k_{0}}^{0}(\xi)$. Then, the symbol $a_{k_{0}}$ satisfies the following properties: 
(i) For $\gamma=1,2$, we have

$$
\left|\partial_{\xi}^{\gamma} a_{k_{0}}(\xi)\right| \lesssim a_{k_{0}}(\xi) \cdot\left(M^{2}+\xi^{2}\right)^{-\gamma / 2} .
$$

(ii) For $|\xi| \leqslant M / 2$ and $k_{0} \in \mathbb{Z}_{M}$, we have

$$
a_{k_{0}}(\xi)=a_{k_{0}}(0) \sim M^{2 s+\delta_{0}} 2^{-\delta_{0} k_{0}} .
$$

(iii) For $\xi \in I_{k}^{M}$, we have

$$
a_{k_{0}}(\xi) \sim 2^{2 k s} 2^{-\delta_{0}\left|k-k_{0}\right|} .
$$

As a consequence of (ii) and (iii), we have, for $|\xi| \sim\left|\xi^{\prime}\right|$,

$$
a_{k_{0}}(\xi) \sim a_{k_{0}}\left(\xi^{\prime}\right) .
$$

Next, we define a sequence $\left\{E_{k_{0}}\right\}_{k_{0} \in \mathbb{Z}_{M}}$ of energy functionals by

$$
E_{k_{0}}(u)(t)=\left\langle a_{k_{0}}(D) u(t), u(t)\right\rangle_{L^{2}}=\sum_{n \in \mathbb{Z}} a_{k_{0}}(n)|\widehat{u}(n, t)|^{2} .
$$

Then, from (2.12) and (2.15), we have

$$
2^{2 k_{0} s}\left\|\mathbf{P}_{k_{0}} u(t)\right\|_{L^{2}(\mathbb{T})}^{2} \lesssim E_{k_{0}}(u)(t)
$$

In particular, from (2.10) and (2.17), we have

$$
\|u\|_{E_{M}^{s}(T)}^{2} \lesssim \sum_{k_{0} \in \mathbb{Z}_{M}} \sup _{t_{k_{0}} \in[-T, T]} E_{k_{0}}(u)\left(t_{k_{0}}\right)
$$

for any $T>0$. In Section 5, we establish the desired energy estimate (1.11) by estimating $\sup _{t_{k_{0}} \in[-T, T]} E_{k_{0}}(u)\left(t_{k_{0}}\right)$ in a summable manner over $k_{0} \in \mathbb{Z}_{M}$.

2.4. On the choice of $\boldsymbol{\alpha}$. In Section 2.2, we defined the function spaces $F_{M}^{s, \alpha}(T)$ and $N_{M}^{s, \alpha}(T)$ depending on a parameter $\alpha>0$. In this subsection, we provide a heuristic discussion on how to choose $\alpha>0$. In fact, we choose the smallest $\alpha>0$ so that a solution to (1.5) localized around the spatial frequencies $\left\{|n| \sim 2^{k}\right\}$ behaves like a linear solution up to time $\sim 2^{-\alpha k}$. Namely, $2^{-\alpha k}$ is the first time scale on which the nonlinear effect becomes visible. In the following, we set $M=1$ for simplicity.

Fix $s<0$ and $k \in \mathbb{Z}_{\geqslant 0}$. Let $f \in L^{2}(\mathbb{T})$ with supp $\widehat{f} \subset I_{k}$ such that $\|f\|_{H^{s}}=1$. Then, we have $\|f\|_{L^{2}} \sim 2^{-k s}$. Let $u$ be the (smooth) solution to (1.5) with $\left.u\right|_{t=0}=$ $f$, satisfying the following Duhamel formulation:

$$
u(t)=S(t) f-i \int_{0}^{t} S\left(t-t^{\prime}\right) \mathfrak{N}(u)\left(t^{\prime}\right) d t^{\prime},
$$


where $\mathfrak{N}(u)$ is the nonlinear part of (1.5) defined in (1.6). We investigate the largest time scale $T$ such that $u(t) \approx S(t) f$ on $[0, T]$. By the standard $X^{s, b}$ estimates and the $L^{4}$-Strichartz estimate (1.3) as in [36], we have

$$
\begin{aligned}
\left\|\int_{0}^{t} S\left(t-t^{\prime}\right) \mathfrak{N}(u)\left(t^{\prime}\right) d t^{\prime}\right\|_{L_{T}^{\infty} L_{x}^{2}} & \lesssim\left\|\int_{0}^{t} S\left(t-t^{\prime}\right) \mathfrak{N}(u)\left(t^{\prime}\right) d t^{\prime}\right\|_{X_{T}^{0,1 / 2+}} \\
& \lesssim\left\||u|^{2} u\right\|_{X_{T}^{0,-1 / 2+}} \\
& =\left.\sup _{\|v\|_{X^{0,1 / 2-}}=1}\left|\int_{\mathbb{T} \times[0, T]} v\right| u\right|^{2} u d x d t \mid \\
& =\sup _{\|v\|_{X^{0,1 / 2-}}=1}\|v\|_{L_{x, T}^{4}}\|u\|_{L_{x, T}^{4}}^{3} \\
& \lesssim T^{3 / 4-}\|u\|_{X_{T}^{0,1 / 2+}}^{3} .
\end{aligned}
$$

By making a heuristic substitution $u(t) \approx S(t) f$,

$$
\lesssim T^{3 / 4-}\|f\|_{L^{2}}^{3} \sim T^{3 / 4-} 2^{-3 k s} .
$$

Here, $X_{T}^{s, b}$ denotes the local-in-time version of the $X^{s, b}$-space restricted on the time interval $[0, T]$. This shows that the solution $u$ basically propagates linearly on the time scale $T$ if $T^{3 / 4-} 2^{-3 k s} \ll 2^{-k s}$, that is, $T \ll 2^{-\alpha k}$ with

$$
\alpha=-\frac{8 s}{3}+\varepsilon
$$

for some small $\varepsilon>0$. Indeed, the condition (2.19) on $\alpha$ naturally appears in establishing the crucial trilinear estimate. See Section 4.

\section{Strichartz and related multilinear estimates}

In this section, we state and prove certain multilinear Strichartz estimates. While the basic structure of the argument follows closely that in [19], we obtain stronger estimates with simpler proofs thanks to the stronger quartic dispersion.

Recall the following periodic $L^{4}$ - and $L^{6}$-Strichartz estimates:

$$
\|u\|_{L_{x, t}^{4}(\mathbb{T} \times \mathbb{R})} \lesssim\|u\|_{X^{0,5 / 16}} \quad \text { and } \quad\|S(t) \phi\|_{L_{x, t}^{6}(\mathbb{T} \times \mathbb{R})} \leqslant C_{\varepsilon}|I|^{\varepsilon}\|\phi\|_{L^{2}}
$$

for any $\varepsilon>0$, where $\phi$ is a function on $\mathbb{T}$ such that supp $\widehat{\phi}$ is contained in an interval $I$ of length $|I|$. These estimates are essentially due to Bourgain [3]. See [36] for the proof of the $L^{4}$-Strichartz estimate. The $L^{6}$-Strichartz estimate follows from the algebraic identity (2.4) and the divisor counting argument as in [3].

By the Galilean transformation and the transference principle, we have the following estimate; if we assume that supp $\widehat{u} \subset D_{\leqslant j} \cap(I \times \mathbb{R})$ for some interval $I$, 
then we have

$$
\|u\|_{L_{x, t}^{6}} \leqslant C_{\varepsilon}|I|^{\varepsilon} 2^{j / 2}\|u\|_{L_{x, t}^{2}}
$$

for any $\varepsilon>0$. As a corollary to (3.1) and (3.2), we have the following lemma. See [19] for the proofs.

LEMMA 3.1. Let $u_{k_{i}, j_{i}}$ be a function on $\mathbb{T} \times \mathbb{R}$ such that $\operatorname{supp} \widehat{u}_{k_{i}, j_{i}} \subset D_{k_{i}, \leqslant j_{i}}^{M}$. Then, we have

$$
\begin{aligned}
& \left|\int_{\mathbb{T} \times \mathbb{R}} u_{k_{1}, j_{1}} \bar{u}_{k_{2}, j_{2}} u_{k_{3}, j_{3}} \bar{u}_{k_{4}, j_{4}} d x d t\right| \lesssim \prod_{i=1}^{4} 2^{5 j_{i} / 16}\left\|\mathcal{F}\left(u_{k_{i}, j_{i}}\right)\right\|_{\ell_{n}^{2} L_{\tau}^{2}}, \\
& \left|\int_{\mathbb{T} \times \mathbb{R}} u_{k_{1}, j_{1}} \bar{u}_{k_{2}, j_{2}} u_{k_{3}, j_{3}} \bar{u}_{k_{4}, j_{4}} d x d t\right| \lesssim 2^{-j_{1}^{*} / 2} 2^{\varepsilon k_{3}^{*}} \prod_{i=1}^{4} 2^{j_{i} / 2}\left\|\mathcal{F}\left(u_{k_{i}, j_{i}}\right)\right\|_{\ell_{n}^{2} L_{\tau}^{2}}
\end{aligned}
$$

for any $\varepsilon>0$. Here, $j_{i}^{*}$ and $k_{i}^{*}$ denote the decreasing rearrangements of $j_{i}$ and $k_{i}$, $i=1, \ldots, 4$.

As in [19], we can refine the analysis and obtain the following multilinear estimates.

LEMMA 3.2. Let $u_{i}$ be supported in $D_{k_{i}, \leqslant j_{i}}^{M}, i=1,2,3$. Suppose that $2^{k_{1}^{*}} \gg M$. Then, the following estimate holds:

$$
\left\|\mathbf{P}_{k_{4}} \mathcal{N}\left(u_{1}, u_{2}, u_{3}\right)\right\|_{L_{x, t}^{2}} \lesssim 2^{k_{4}^{*} / 2} \min _{i=1,3}\left\{\left(1+2^{j_{i}-2 k_{1}^{*}}\right)^{1 / 2} 2^{-j_{i} / 2}\right\}\left(\prod_{i=1}^{3} 2^{j_{i} / 2}\left\|\mathcal{F}\left(u_{i}\right)\right\|_{\ell_{n}^{2} L_{\tau}^{2}}\right),
$$

Here, $\mathcal{N}\left(u_{1}, u_{2}, u_{3}\right)$ is the nonresonant part of the nonlinearity defined in (1.7).

The proof of Lemma 3.2 is analogous to that of [19, Lemma 5.3]. Note that, thanks to the stronger dispersion, we do not need frequency separation which was assumed in [19, Lemma 5.3].

Proof. Let $f_{i}=\widehat{u}_{i}$ for $i=1,3$ and $f_{i}=\widehat{u}_{i}$ for $i=2$. By duality, we have

LHS of (3.5)

$$
=\sup _{\substack{\left\|f_{4}\right\|_{L^{2}=1} \\ \operatorname{supp} f_{4} \subset I_{k_{4}}^{M} \times \mathbb{R}}} \int_{\tau_{1}-\tau_{2}+\tau_{3}-\tau_{4}=0} \sum_{\substack{n_{1}-n_{2}+n_{3}-n_{4}=0 \\ n_{1} \neq n_{2}, n_{4}}} \prod_{i=1}^{4} f_{i}\left(n_{i}, \tau_{i}\right) d \tau_{1} d \tau_{2} d \tau_{3} .
$$


For simplicity of notations, we drop the supremum over $f_{4}$ in the following. Note that, under the assumption $2^{k_{1}^{*}} \gg M$, we have

$$
n_{1}^{*} \sim 2^{k_{1}^{*}} .
$$

See Remark 3.4 below.

- Case (a): $\left|n_{4}\right| \lesssim 2^{k_{4}^{*}}$.

Under $n_{1}-n_{2}+n_{3}-n_{4}=0$, we have $\max \left\{\left|n_{2}\right|,\left|n_{3}\right|\right\} \sim 2^{k_{1}^{*}}$. With $g_{i}(n, \tau)=$ $f_{i}\left(n, \tau-n^{4}\right)$, we have

$$
\begin{aligned}
(3.6) \leqslant & \int \sum_{n_{4}}\left|g_{4}\left(n_{4}, \tau_{4}\right)\right| \sum_{n_{1}, n_{2}}\left|g_{1}\left(n_{1}, \tau_{1}\right)\right|\left|g_{2}\left(n_{2}, \tau_{2}\right)\right| \\
& \times\left|g_{3}\left(-n_{1}+n_{2}+n_{4}, h_{3}\left(n_{1}, n_{2}, n_{4}, \tau_{1}, \tau_{2}, \tau_{4}\right)\right)\right| d \tau_{1} d \tau_{2} d \tau_{4},
\end{aligned}
$$

where $h_{3}\left(n_{1}, n_{2}, n_{4}, \tau_{1}, \tau_{2}, \tau_{4}\right)$ is defined by

$$
h_{3}\left(n_{1}, n_{2}, n_{4}, \tau_{1}, \tau_{2}, \tau_{4}\right)=-\tau_{1}+\tau_{2}+\tau_{4}+n_{1}^{4}-n_{2}^{4}-n_{4}^{4}+\left(-n_{1}+n_{2}+n_{4}\right)^{4} .
$$

For fixed $n_{1}, n_{4}, \tau_{1}, \tau_{2}$, and $\tau_{4}$, define the set $E_{32}=E_{32}\left(n_{1}, n_{4}, \tau_{1}, \tau_{2}, \tau_{4}\right)$ by

$$
E_{32}=\left\{n_{2} \in \mathbb{Z}: h_{3}\left(n_{1}, n_{2}, n_{4}, \tau_{1}, \tau_{2}, \tau_{4}\right)=O\left(2^{j_{3}}\right)\right\} .
$$

Since $n_{1} \neq n_{4}$, we have

$$
\begin{aligned}
\left|\partial_{n_{2}} h_{3}\right| & =4\left|-n_{2}^{3}+\left(-n_{1}+n_{2}+n_{4}\right)^{3}\right| \\
& =4\left|\left(-n_{1}+n_{4}\right)\left(n_{2}^{2}+n_{2}\left(-n_{1}+n_{2}+n_{4}\right)+\left(-n_{1}+n_{2}+n_{4}\right)^{2}\right)\right| \\
& \gtrsim \max \left\{n_{2}^{2}, n_{3}^{2}\right\} \sim 2^{2 k_{1}^{*}},
\end{aligned}
$$

where the second to the last step follows from completing a square:

$$
n_{2}^{2}+n_{2} n_{3}+n_{3}^{2}=\left(n_{2}+\frac{1}{2} n_{3}\right)^{2}+\frac{3}{4} n_{3}^{2}=\frac{3}{4} n_{2}^{2}+\left(\frac{1}{2} n_{2}+n_{3}\right)^{2} .
$$

Hence, we conclude that

$$
\left|E_{32}\right| \lesssim 1+2^{j_{3}-2 k_{1}^{*}}
$$

Now we are ready to estimate (3.6). By Cauchy-Schwarz inequality in $n_{2}, n_{1}$, $n_{4}$, we obtain

$$
\begin{aligned}
(3.7) \lesssim & \left(1+2^{j_{3}-2 k_{1}^{*}}\right)^{1 / 2} \int \sum_{n_{4}}\left|g_{4}\left(n_{4}, \tau_{4}\right)\right| \sum_{n_{1}}\left|g_{1}\left(n_{1}, \tau_{1}\right)\right|\left(\sum_{n_{2}}\left|g_{2}\left(n_{2}, \tau_{2}\right)\right|^{2}\right. \\
& \left.\times\left|g_{3}\left(-n_{1}+n_{2}+n_{4}, h_{3}\left(n_{1}, n_{2}, n_{4}, \tau_{1}, \tau_{2}, \tau_{4}\right)\right)\right|^{2}\right)^{1 / 2} d \tau_{1} d \tau_{2} d \tau_{4}
\end{aligned}
$$




$$
\begin{aligned}
\lesssim & 2^{k_{4} / 2} \cdot\left(1+2^{j_{3}-2 k_{1}^{*}}\right)^{1 / 2}\left\|g_{4}\left(n_{4}, \tau_{4}\right)\right\|_{\ell_{n_{4}}^{2} L_{\tau_{4}}^{2}} \\
& \times \sup _{n_{4}} \int\left\|g_{1}\left(n_{1}, \tau_{1}\right)\right\|_{\ell_{n_{1}}^{2}}\left(\sum_{n_{2}}\left|g_{2}\left(n_{2}, \tau_{2}\right)\right|^{2}\right. \\
& \left.\times \sum_{n_{1}}\left\|g_{3}\left(-n_{1}+n_{2}+n_{4}, h_{3}\left(n_{1}, n_{2}, n_{4}, \tau_{1}, \tau_{2}, \tau_{4}\right)\right)\right\|_{L_{\tau_{4}}^{2}}^{2}\right)^{1 / 2} d \tau_{1} d \tau_{2} .
\end{aligned}
$$

Noting that $h_{3}$ is linear in $\tau_{4}$ and applying Cauchy-Schwarz inequality in $\tau_{1}$ and $\tau_{2}$,

$$
\begin{aligned}
& \leqslant 2^{k_{4} / 2} \cdot\left(1+2^{j_{3}-2 k_{1}^{*}}\right)^{1 / 2}\left(\prod_{i_{1}=1}^{2}\left\|g_{i_{1}}\right\|_{L_{\tau_{i_{1}}}^{1} \ell_{n_{i_{1}}}^{2}}\right)\left(\prod_{i_{2}=3}^{4}\left\|g_{i_{2}}\right\|_{\ell_{n_{i_{2}}}^{2} L_{\tau_{i_{2}}}^{2}}\right) \\
& \lesssim 2^{k_{4} / 2} \cdot\left(1+2^{j_{3}-2 k_{1}^{*}}\right)^{1 / 2} 2^{-j_{3} / 2} \prod_{i=1}^{3} 2^{j_{i} / 2}\left\|f_{i}\right\|_{\ell_{n}^{2} L_{\tau}^{2}},
\end{aligned}
$$

yielding (3.5). Note that even if we replace the role of $n_{1}$ and $n_{3}$, the same argument still holds with a factor $\left(1+2^{j_{1}-2 k_{1}^{*}}\right)^{1 / 2} 2^{-j_{1} / 2}$. The same comment applies to Cases (b) and (c).

- Case (b): $\left|n_{1}\right| \sim 2^{k_{4}^{*}}$. (A similar argument applies to the case $\left|n_{3}\right| \sim 2^{k_{4}^{*}}$.)

In this case, we have $\max \left\{\left|n_{2}\right|,\left|n_{3}\right|\right\} \sim 2^{k_{1}^{*}}$ and thus (3.9) holds. Then, proceeding as before, we have

$$
\begin{aligned}
(3.7) \lesssim & \left(1+2^{j_{3}-2 k_{1}^{*}}\right)^{1 / 2} \int \sum_{n_{1}}\left|g_{1}\left(n_{1}, \tau_{1}\right)\right| \sum_{n_{4}}\left|g_{4}\left(n_{4}, \tau_{4}\right)\right|\left(\sum_{n_{2}}\left|g_{2}\left(n_{2}, \tau_{2}\right)\right|^{2}\right. \\
& \left.\times\left|g_{3}\left(-n_{1}+n_{2}+n_{4}, h_{3}\left(n_{1}, n_{2}, n_{4}, \tau_{1}, \tau_{2}, \tau_{4}\right)\right)\right|^{2}\right)^{1 / 2} d \tau_{1} d \tau_{2} d \tau_{4} \\
\lesssim & 2^{k_{1} / 2} \cdot\left(1+2^{j_{3}-2 k_{1}^{*}}\right)^{1 / 2} \int\left\|g_{1}\left(n_{1}, \tau_{1}\right)\right\|_{\ell_{n_{1}}^{2}} d \tau_{1}\left\|g_{4}\left(n_{4}, \tau_{4}\right)\right\|_{\ell_{n_{4}}^{2} L_{\tau_{4}}^{2}} \\
& \times \sup _{n_{1}, \tau_{1}} \int\left(\sum_{n_{2}}\left|g_{2}\left(n_{2}, \tau_{2}\right)\right|^{2}\right. \\
& \left.\times \sum_{n_{4}}\left\|g_{3}\left(-n_{1}+n_{2}+n_{4}, h_{3}\left(n_{1}, n_{2}, n_{4}, \tau_{1}, \tau_{2}, \tau_{4}\right)\right)\right\|_{L_{\tau_{4}}^{2}}^{2}\right)^{1 / 2} d \tau_{2} .
\end{aligned}
$$

The rest follows as in Case (a). 
- Case (c): $k_{2}=k_{4}^{*}$.

In this case, we have $\max \left\{\left|n_{1}\right|,\left|n_{3}\right|\right\} \sim 2^{k_{1}^{*}}$. For fixed $n_{2}, n_{4}, \tau_{1}, \tau_{2}$, and $\tau_{4}$, define the set $E_{31}=E_{31}\left(n_{2}, n_{4}, \tau_{1}, \tau_{2}, \tau_{4}\right)$ by

$$
E_{31}=\left\{n_{1} \in \mathbb{Z}: h_{3}\left(n_{1}, n_{2}, n_{4}, \tau_{1}, \tau_{2}, \tau_{4}\right)=O\left(2^{j_{3}}\right)\right\},
$$

where $h_{3}$ is as in (3.8). Note that

$$
\begin{aligned}
\left|\partial_{n_{1}} h\right| & =4\left|n_{1}^{3}-4\left(-n_{1}+n_{2}+n_{4}\right)^{3}\right| \\
& =4\left|\left(2 n_{1}-n_{2}-n_{4}\right)\left(n_{1}^{2}+n_{1}\left(-n_{1}+n_{2}+n_{4}\right)+\left(-n_{1}+n_{2}+n_{4}\right)^{2}\right)\right| \\
& \sim\left|n_{1}-n_{3}\right| \cdot 2^{2 k_{1}^{*}} .
\end{aligned}
$$

If $n_{1}=n_{3}$, then we have $n_{1}=\left(n_{2}+n_{4}\right) / 2$. Namely, $n_{1}$ is uniquely determined for fixed $n_{2}$ and $n_{4}$ and hence we have $\left|E_{31}\right|=1$. Otherwise, we have $\left|\partial_{n_{1}} h\right| \gtrsim 2^{2 k_{1}^{*}}$. Therefore, we conclude that

$$
\left|E_{31}\right| \lesssim 1+2^{j_{3}-2 k_{1}^{*}}
$$

Then, proceeding as before, we have

$$
\begin{aligned}
(3.7) \lesssim & \left(1+2^{j_{3}-2 k_{1}^{*}}\right)^{1 / 2} \int \sum_{n_{4}}\left|g_{4}\left(n_{4}, \tau_{4}\right)\right| \sum_{n_{2}}\left|g_{2}\left(n_{2}, \tau_{2}\right)\right|\left(\sum_{n_{1}}\left|g_{1}\left(n_{1}, \tau_{1}\right)\right|^{2}\right. \\
& \left.\times\left|g_{3}\left(-n_{1}+n_{2}+n_{4}, h_{3}\left(n_{1}, n_{2}, n_{4}, \tau_{1}, \tau_{2}, \tau_{4}\right)\right)\right|^{2}\right)^{1 / 2} d \tau_{1} d \tau_{2} d \tau_{4} \\
\lesssim & 2^{k_{2} / 2} \cdot\left(1+2^{j_{3}-2 k_{1}^{*}}\right)^{1 / 2} \int\left\|g_{2}\left(n_{2}, \tau_{2}\right)\right\|_{\ell_{n_{2}}} d \tau_{2}\left\|g_{4}\left(n_{4}, \tau_{4}\right)\right\|_{\ell_{n_{4}}^{2} L_{\tau_{4}}^{2}} \\
& \times \sup _{n_{2}, \tau_{2}} \int\left(\sum_{n_{1}}\left|g_{1}\left(n_{1}, \tau_{1}\right)\right|^{2}\right. \\
& \left.\times \sum_{n_{4}}\left\|g_{3}\left(-n_{1}+n_{2}+n_{4}, h_{3}\left(n_{1}, n_{2}, n_{4}, \tau_{1}, \tau_{2}, \tau_{4}\right)\right)\right\|_{L_{\tau_{4}}^{2}}^{2}\right)^{1 / 2} d \tau_{1} .
\end{aligned}
$$

Then, the rest follows as before.

As a corollary to Lemma 3.2, we obtain the following multilinear estimates by further assuming $j_{i} \geqslant\left[\alpha k_{1}^{*}\right], i=1,2,3$, and $\alpha \in[0,2]$.

LEMMA 3.3. Let $\alpha \in[0,2]$. Let $u_{i}$ be a function on $\mathbb{T} \times \mathbb{R}$ such that $\operatorname{supp} \widehat{u}_{i} \subset$ $D_{k_{i}, j_{i}}^{M}$ and $2^{k_{1}^{*}} \gg M$. Suppose that $j_{1}, j_{2}, j_{3} \geqslant\left[\alpha k_{1}^{*}\right]$. Then, we have

$$
\left|\int_{\mathbb{T} \times \mathbb{R}} \mathcal{N}\left(u_{1}, u_{2}, u_{3}\right) \cdot \bar{u}_{4} d x d t\right| \lesssim 2^{-j_{1}^{*} / 2} 2^{-(1 / 2) \alpha k_{1}^{*}+(1 / 2) k_{4}^{*}} \prod_{i=1}^{4} 2^{j_{i} / 2}\left\|\mathcal{F}\left(u_{i}\right)\right\|_{\ell_{n}^{2} L_{\tau}^{2}} .
$$


When $j_{4} \gtrsim j_{1}^{*}$, by noting that $2^{-j_{4} / 2} \lesssim 2^{-j_{1}^{*} / 2}$, the desired estimate (3.10) directly follows from Lemma 3.2. When $j_{4} \ll j_{1}^{*}$, we first rewrite the left-hand side of (3.10) as

$$
\left|\int_{\mathbb{T} \times \mathbb{R}} \mathcal{N}\left(u_{1}, u_{2}, u_{3}\right) \cdot \bar{u}_{4} d x d t\right|=\left|\int_{\mathbb{T} \times \mathbb{R}} \mathcal{N}\left(u_{i_{1}}, u_{i_{2}}, u_{i_{3}}\right) \cdot \bar{u}_{i_{4}} d x d t\right|,
$$

where $\left(i_{1}, i_{2}, i_{3}, i_{4}\right)=(2,3,4,1),(3,4,1,2)$, or $(4,1,2,3)$ such that $j_{i_{4}} \gtrsim j_{1}^{*}$. Then, (3.10) in this case also follows from Lemma 3.2.

REMARK 3.4. The assumption $2^{k_{1}^{*}} \gg M$ is necessary in Lemmas 3.2 and 3.3. In fact, when $2^{k_{1}^{*}}=M$, we only know that $n_{1}^{*}$ belongs to the interval $I_{\text {low }}^{M}$ but it is possible to have $n_{1}^{*} \ll 2^{k_{1}^{*}}=M$. We also point out that Lemmas 3.2 and 3.3 also hold under an alternative assumption: $n_{1}^{*} \sim 2^{k_{1}^{*}}$. This observation plays an important role in the energy estimate in Section 5, where we apply symmetrization to eliminate the contribution from the low frequencies $\left\{\left(n_{1}, n_{2}, n_{3}, n_{4}\right): n_{1}^{*} \leqslant\right.$ $M / 2\}$.

We conclude this section by stating a multilinear estimate when there is a gap between the two largest (spatial) frequencies and the rest.

LEMMA 3.5. Let $\alpha \in[0,2]$. Let $u_{i}$ be a function on $\mathbb{T} \times \mathbb{R}$ such that $\operatorname{supp} \widehat{u}_{i} \subset$ $D_{k_{i}, j_{i}}^{M}$. Suppose that $k_{3}, k_{4} \leqslant k_{2}^{*}-10, j_{1}, j_{2}, j_{3} \geqslant\left[\alpha k_{1}^{*}\right]$, and $2^{j_{1}^{*}} \geqslant|\phi(\bar{n})|$, where $\phi(\bar{n})$ is the phase function defined in (2.4). Then, we have

$$
\left|\int_{\mathbb{T} \times \mathbb{R}} \mathcal{N}\left(u_{1}, u_{2}, u_{3}\right) \cdot \bar{u}_{4} d x d t\right| \lesssim \Lambda \cdot \prod_{i=1}^{4} 2^{j_{i} / 2}\left\|\mathcal{F}\left(u_{i}\right)\right\|_{\ell_{n}^{2} L_{\tau}^{2}},
$$

where $\Lambda$ is given by

$$
\Lambda= \begin{cases}2^{-(1 / 2)(3+\alpha) k_{1}^{*}-k_{3}^{*} / 2+k_{4}^{*} / 2} & \text { if }\left|k_{3}-k_{4}\right| \geqslant 2, \\ 2^{-(1 / 2)(3+\alpha) k_{1}^{*}+k_{3}^{*} / 4} & \text { otherwise. }\end{cases}
$$

Proof. First, we consider the case $\left|k_{3}-k_{4}\right| \geqslant 2$. Then, we have $j_{1}^{*} \geqslant 3 k_{1}^{*}+k_{3}^{*}-5$, since $|\phi(\bar{n})| \sim\left|\left(n_{2}-n_{3}\right)\left(n_{3}-n_{4}\right)\right|\left(n_{1}^{*}\right)^{2} \sim\left(n_{1}^{*}\right)^{3}\left|n_{3}-n_{4}\right| \sim 2^{3 k_{1}^{*}}\left|n_{3}-n_{4}\right|$. Then, (3.11) follows from Lemma 3.3. Here we used $n_{1}^{*} \sim 2^{k_{1}^{*}}$ and $n_{3}^{*} \sim 2^{k_{3}^{*}}$, which was implied by the assumption $2^{k_{1}^{*}} \gg 2^{k_{3}^{*}} \gg 2^{k_{4}^{*}} \geqslant M$.

Next, we consider the case $\left|k_{3}-k_{4}\right| \leqslant 1$. We separately estimate the contributions from the following two cases: (a) $\left|n_{3}-n_{4}\right| \geqslant 2^{k_{3}^{*} / 2}$ and (b) $\left|n_{3}-n_{4}\right| \leqslant 2^{k_{3}^{*} / 2}$. 
In Case (a), we have $j_{1}^{*} \geqslant(3 / 2) k_{1}^{*}+k_{3}^{*} / 2-5$. Then, Lemma 3.3 yields

$$
\text { LHS of }(3.11) \lesssim 2^{-(1 / 2)(3+\alpha) k_{1}^{*}} 2^{k_{3}^{*} / 4} \prod_{i=1}^{4} 2^{j_{i} / 2}\left\|\mathcal{F}\left(u_{i}\right)\right\|_{\ell_{n}^{2} L_{\tau}^{2}} .
$$

In Case (b), we write $I_{k_{3}}=\bigcup_{\ell_{i}} J_{\ell_{i}}, i=3,4$ where $\left|J_{\ell_{i}}\right|=2^{k_{3}^{*} / 2}$. Then, if $n_{3} \in J_{\ell_{3}}$ for some $\ell_{3}$, there are only $O(1)$ many possible values of $\ell_{4}=\ell_{4}\left(\ell_{3}\right)$ such that $n_{4} \in J_{\ell_{4}}$. Then, by writing

$$
\sum_{n_{3}} \sum_{n_{4}}=\sum_{\ell_{3}} \sum_{\ell_{4}=\ell_{4}\left(\ell_{3}\right)} \sum_{n_{3} \in J_{\ell_{3}}} \sum_{n_{4} \in J_{\ell_{4}}}
$$

and repeating the previous argument for each $\ell_{3}$, we only lose $\left|J_{\ell_{i}}\right|^{1 / 2}=2^{k_{3}^{*} / 4}$ by applying Cauchy-Schwarz inequality in $n_{3}$ or $n_{4}$ at the end. Finally, applying Cauchy-Schwarz inequality in $\ell_{3}$, we obtain (3.12).

\section{Trilinear estimates}

In this section, we prove the crucial trilinear estimate for the Wick ordered cubic 4NLS (1.5). This establishes the nonlinear estimate part (1.10) of the short-time Fourier restriction norm method.

Proposition 4.1. Let $s \in\left(-\frac{9}{20}, 0\right)$ and $T>0$. Then, with $\alpha=-8 s / 3+$, there exists $\theta>0$ such that

$$
\left\|\mathcal{N}\left(u_{1}, u_{2}, u_{3}\right)\right\|_{N_{M}^{s, \alpha}(T)}+\left\|\mathcal{R}\left(u_{1}, u_{2}, u_{3}\right)\right\|_{N_{M}^{s, \alpha}(T)} \lesssim T^{\theta} \prod_{i=1}^{3}\left\|u_{i}\right\|_{F_{M}^{s, \alpha}(T)},
$$

where $\mathcal{N}\left(u_{1}, u_{2}, u_{3}\right)$ and $\mathcal{R}\left(u_{1}, u_{2}, u_{3}\right)$ are as in (1.7) and (1.8).

The proof of Proposition 4.1 is analogous to the proof of the trilinear estimate for the Wick ordered cubic NLS (1.12) considered in [19]. More precisely, we prove Proposition 4.1 by first applying the dyadic decomposition and then performing case-by-case analysis on different frequency interactions. For readers' convenience, we first summarize the size estimates on the phase function $\phi(\bar{n})$ defined in (2.4) in various frequency regimes under the nonresonance assumption $\left\{n_{1}, n_{3}\right\} \neq\left\{n, n_{2}\right\}$ :

(i) If $|n| \sim\left|n_{3}\right| \gg\left|n_{1}\right|,\left|n_{2}\right|$, then

$$
|\phi(\bar{n})| \sim\left(n_{1}^{*}\right)^{3}\left|n_{2}-n_{1}\right| .
$$


(ii) If $|n| \sim\left|n_{2}\right| \gg\left|n_{1}\right|,\left|n_{3}\right|$, then

$$
|\phi(\bar{n})| \sim\left(n_{1}^{*}\right)^{4} .
$$

(iii) If $|n| \sim\left|n_{2}\right| \sim\left|n_{3}\right| \gg\left|n_{1}\right|$, then

$$
|\phi(\bar{n})| \sim\left(n_{1}^{*}\right)^{4} .
$$

These size estimates immediately follow from the factorization in (2.4). Note that the conditions (i)-(iii) hold under the symmetries $n_{1} \leftrightarrow n_{3}$ and $n \leftrightarrow n_{2}$, and $\left\{n_{1}\right.$, $\left.n_{3}\right\} \leftrightarrow\left\{n, n_{2}\right\}$, respectively. Recall that we have $2^{k_{i}} \geqslant M, i=1, \ldots, 4$.

In the following, by assuming that $u_{i}$ has the Fourier transform supported on $I_{k_{i}} \times \mathbb{R}$, we prove trilinear estimates for different frequency interactions. We first consider the case when the output frequency is high (relative to the input frequencies). In particular, this also includes the case when all the frequencies are low.

LEMMA 4.2. Let $\alpha \geqslant 0$. If $k_{4} \geqslant k_{1}^{*}-5$, then we have

$$
\left\|\mathbf{P}_{k_{4}} \mathcal{N}\left(u_{1}, u_{2}, u_{3}\right)\right\|_{N_{M, k_{4}}^{\alpha}}+\left\|\mathbf{P}_{k_{4}} \mathcal{R}\left(u_{1}, u_{2}, u_{3}\right)\right\|_{N_{M, k_{4}}^{\alpha}} \lesssim 2^{-(3 / 4)(\alpha-\varepsilon) k_{1}^{*}} \prod_{i=1}^{3}\left\|u_{i}\right\|_{F_{M, k_{i}}^{\alpha}}
$$

for any $\varepsilon>0$.

In view of (1.8), there is no contribution from the resonant part $\mathcal{R}\left(u_{1}, u_{2}, u_{3}\right)$ except for the case: $2^{k_{1}^{*}} \sim 2^{k_{4}^{*}}$, which is treated in Lemma 4.2. Lemma 4.2 also handles the low frequency case: $2^{k_{1}^{*}} \lesssim M$. The proof of Lemma 4.2 closely follows that of [19, Lemma 6.2]. We present the details for readers' convenience.

Proof. Let $\gamma: \mathbb{R} \rightarrow[0,1]$ be a smooth cutoff function supported on $[-1,1]$ with $\gamma \equiv 1$ on $\left[-\frac{1}{4}, \frac{1}{4}\right]$ such that

$$
\sum_{m \in \mathbb{Z}} \gamma^{3}(t-m) \equiv 1, \quad t \in \mathbb{R}
$$

Then, there exist $c, C>0$ such that

$$
\eta_{0}\left(2^{\left[\alpha k_{4}\right]}\left(t-t_{k_{4}}\right)\right)=\eta_{0}\left(2^{\left[\alpha k_{4}\right]}\left(t-t_{k_{4}}\right)\right) \sum_{|m| \leqslant C} \gamma^{3}\left(2^{\left[\alpha k_{1}^{*}\right]+c}\left(t-t_{k_{4}}\right)-m\right)
$$

and

$$
\eta_{0}\left(2^{\left[\alpha k_{i}\right]} t\right) \cdot \gamma\left(2^{\left[\alpha k_{1}^{*}\right]+c} t\right)=\gamma\left(2^{\left[\alpha k_{1}^{*}\right]+c} t\right)
$$


for $i=1,2,3$. Let $f_{k_{i}}=\mathcal{F}\left[\gamma\left(2^{\left[\alpha k_{1}^{*}\right]+c}\left(t-t_{k_{4}}\right)\right) \cdot u_{i}\right], i=1,3$, and $\overline{f_{k_{2}}}=$ $\mathcal{F}\left[\gamma\left(2^{\left[\alpha k_{1}^{*}\right]+c}\left(t-t_{k_{4}}\right)\right) \cdot u_{2}\right]$. Then, it follows from the definition and Lemma 2.1 that

$$
\begin{aligned}
& \text { LHS of (4.4) } \lesssim \sup _{t_{k_{4}} \in \mathbb{R}}\left\|\left(\tau+n^{4}+i 2^{\left[\alpha k_{4}\right]}\right)^{-1} \mathbf{1}_{I_{k_{4}}}(n)\left(f_{k_{1}} * \widetilde{f}_{k_{2}} * f_{k_{3}}\right)\right\|_{X_{M, k_{4}}} \\
& \lesssim \sup _{t_{k_{4}} \in \mathbb{R}} \sum_{j_{4}=0}^{\infty} 2^{j_{4} / 2} \sum_{j_{1}, j_{2}, j_{3} \geqslant\left[\alpha k_{4}\right]} \|\left(2^{j_{4}}+2^{\left[\alpha k_{4}\right]}\right)^{-1} \\
& \times \mathbf{1}_{D_{k_{4}, j_{4}}^{M}} \cdot\left(f_{k_{1}, j_{1}} * \widetilde{f}_{k_{2}, j_{2}} * f_{k_{3}, j_{3}}\right) \|_{\ell_{n}^{2} L_{\tau}^{2}},
\end{aligned}
$$

where $\tilde{f}(n, \tau)=f(-n,-\tau)$ and $f_{k_{i}, j_{i}}, i=1,2,3$, is defined by

$$
f_{k_{i}, j_{i}}(n, \tau)= \begin{cases}f_{k_{i}}(n, \tau) \eta_{j_{i}}\left(\tau+n^{4}\right) & \text { for } j_{i}>\left[\alpha k_{4}\right], \\ f_{k_{i}}(n, \tau) \eta_{\leqslant\left[\alpha k_{4}\right]}\left(\tau+n^{4}\right) & \text { for } j_{i}=\left[\alpha k_{4}\right] .\end{cases}
$$

Using the fact $\mathbf{1}_{D_{k_{4}, j_{4}}^{M}} \leqslant \mathbf{1}_{D_{k_{4}, \leqslant j_{4}}^{M}}$, we have

$$
\begin{aligned}
\text { (4.6) } \lesssim & \sup _{t_{k_{4}} \in \mathbb{R}}\left(\sum_{j_{4}<\left[\alpha k_{4}\right]}+\sum_{j_{4} \geqslant\left[\alpha k_{4}\right]}\right) 2^{j_{4} / 2} \\
& \times \sum_{j_{1}, j_{2}, j_{3} \geqslant\left[\alpha k_{4}\right]}\left\|\left(2^{j_{4}}+2^{\left[\alpha k_{4}\right]}\right)^{-1} \mathbf{1}_{D_{k_{4}, j_{4}}^{M}} f_{k_{1}, j_{1}} * \widetilde{f}_{k_{2}, j_{2}} * f_{k_{3}, j_{3}}\right\|_{\ell_{n}^{2} L_{\tau}^{2}} \\
\lesssim & \sup _{t_{k_{4}} \in \mathbb{R}} \sum_{j_{1}, j_{2}, j_{3}, j_{4} \geqslant\left[\alpha k_{4}\right]} 2^{-j_{4} / 2}\left\|\mathbf{1}_{D_{k_{4}, \leqslant j_{4}}^{M}} \cdot\left(f_{k_{1}, j_{1}} * \widetilde{f}_{k_{2}, j_{2}} * f_{k_{3}, j_{3}}\right)\right\|_{\ell_{n}^{2} L_{\tau}^{2}} \\
\lesssim & \sup _{t_{k_{4}} \in \mathbb{R}} \sup _{j_{4} \geqslant\left[\alpha k_{4}\right]} \sum_{j_{1}, j_{2}, j_{3} \geqslant\left[\alpha k_{4}\right]} 2^{-j_{4} / 2-}\left\|\mathbf{1}_{D_{k_{4}, \leqslant j_{4}}^{M}} \cdot\left(f_{k_{1}, j_{1}} * \widetilde{f}_{k_{2}, j_{2}} * f_{k_{3}, j_{3}}\right)\right\|_{\ell_{n}^{2} L_{\tau}^{2}} .
\end{aligned}
$$

Then, (4.4) follows from (3.3) in Lemma 3.1 and (2.8) with (4.5).

REMARK 4.3. In the proof of Lemma 4.2, we used the $L^{4}$-Strichartz estimate (3.3) in Lemma 3.1. We point out that the multilinear Strichartz estimates in Lemmas 3.2 and 3.3 do not yield a better bound in this case. Consider the case: high $\times$ high $\times$ high $\rightarrow$ high. Then, applying Lemma 3.3 to (4.7) yields a bound with a constant $\sim 2^{(-\alpha+1 / 2+\varepsilon) k_{1}^{*}}$, which is worse than the constant $2^{-(3 / 4)(\alpha-\varepsilon) k_{1}^{*}}$ in (4.4) when $\alpha \leqslant 2$. The proof of Lemma 4.2 based on the $L^{4}$-Strichartz estimate (3.3) in Lemma 3.1 also allows us to handle the case: $2^{k_{1}^{*}} \leqslant M$, for which Lemma 3.3 is not applicable. See Remark 3.4. 
Next, we consider the case when the output frequency is low relative to the input frequencies. In such a case, we have $2^{k_{1}^{*}} \gg 2^{k_{4}} \geqslant M$. We treat this case in the next two lemmas.

LEMmA 4.4 (High $\times$ high $\times$ high $\rightarrow$ low). Let $\alpha \geqslant 0$. If $k_{3} \geqslant \max \left(20, \log _{2} M\right)$, $\left|k_{3}-k_{i}\right| \leqslant 5, i=1,2$, and $k_{4} \leqslant k_{1}-10$, then we have

$$
\left\|\mathbf{P}_{k_{4}} \mathcal{N}\left(u_{1}, u_{2}, u_{3}\right)\right\|_{N_{M, k_{4}}^{\alpha}} \lesssim \min \left(\Lambda_{1}, \Lambda_{2}\right)\left\|u_{1}\right\|_{F_{M, k_{1}}^{\alpha}}\left\|u_{2}\right\|_{F_{M, k_{2}}^{\alpha}}\left\|u_{3}\right\|_{F_{M, k_{3}}^{\alpha}}
$$

where $\Lambda_{1}$ and $\Lambda_{2}$ are given by

$$
\Lambda_{1}=2^{(-2+\alpha+\varepsilon) k_{1}^{*}-\alpha k_{4}} \quad \text { and } \quad \Lambda_{2}=2^{(-2+\alpha / 2+\varepsilon) k_{1}^{*}+(1 / 2-\alpha) k_{4}}
$$

for any $\varepsilon>0$.

Proof. In this case, we localize each component function $u_{i}$ onto subintervals of length $\sim 2^{-\alpha k_{1}^{*}}$. With $\gamma: \mathbb{R} \rightarrow[0,1]$ as in the proof of Lemma 4.2, we have

$$
\eta_{0}\left(2^{\left[\alpha k_{4}\right]}\left(t-t_{k_{4}}\right)\right)=\eta_{0}\left(2^{\left[\alpha k_{4}\right]}\left(t-t_{k_{4}}\right)\right) \sum_{|m| \leqslant C 2^{\left[\alpha k_{1}^{*}\right]-\left[\alpha k_{4}\right]}} \gamma^{3}\left(2^{\left[\alpha k_{1}^{*}\right]+c}\left(t-t_{k_{4}}\right)-m\right)
$$

and $\eta_{0}\left(2^{\left[\alpha k_{i}\right]} t\right) \cdot \gamma\left(2^{\left[\alpha k_{1}\right]+c} t\right)=\gamma\left(2^{\left[\alpha k_{1}\right]+c} t\right)$ for $i=1,2$, 3. In particular, we divide the time interval of length $\sim 2^{-\alpha k_{4}}$ into $O\left(2^{\alpha\left(k_{1}^{*}-k_{4}\right)}\right)$ many subintervals of length $\sim 2^{-\alpha k_{1}^{*}}$. Then, proceeding as in the proof of Lemma 4.2 with (2.8), it suffices to prove that

$$
\begin{aligned}
2^{\alpha\left(k_{1}^{*}-k_{4}\right)} & \sum_{j_{4} \geqslant\left[\alpha k_{4}\right]} 2^{-j_{4} / 2}\left\|\mathbf{1}_{\widetilde{D}_{k_{4}, j_{4}}^{M}} \cdot\left(f_{k_{1}, j_{1}} * \widetilde{f}_{k_{2}, j_{2}} * f_{k_{3}, j_{3}}\right)\right\|_{\ell_{n}^{2} L_{\tau}^{2}} \\
& \lesssim \min \left(\Lambda_{1}, \Lambda_{2}\right) \prod_{i=1}^{3} 2^{j_{i} / 2}\left\|f_{k_{i}, j_{i}}\right\|_{\ell_{n}^{2} L_{\tau}^{2}}
\end{aligned}
$$

for any $f_{k_{i}, j_{i}}: \mathbb{Z} \times \mathbb{R} \rightarrow \mathbb{R}_{+}$supported on $\widetilde{D}_{k_{i}, j_{i}}^{M}$ with $j_{i} \geqslant\left[\alpha k_{1}^{*}\right], i=1,2,3$, where

$$
\widetilde{D}_{k_{i}, j_{i}}^{M}= \begin{cases}D_{k_{i}, \leqslant j_{i}}^{M} & \text { when } j_{i}=\left[\alpha k_{1}^{*}\right] \\ D_{k_{i}, j_{i}}^{M} & \text { when } j_{i}>\left[\alpha k_{1}^{*}\right]\end{cases}
$$

Here, we can assume that $j_{i} \geqslant\left[\alpha k_{1}^{*}\right], i=1,2,3$, thanks to the time localization over an interval of size $\sim 2^{-\left[\alpha k_{1}^{*}\right]}$ and (2.8). Hence, (4.8) with $\Lambda_{1}$ follows from (3.4) in Lemma 3.1 with (4.3), while (4.8) with $\Lambda_{2}$ follows from Lemma 3.3. 
LEMMA 4.5 (High $\times$ high $\times$ low $\rightarrow$ low). Let $\alpha \in[0,2]$. If $k_{1} \geqslant 20,\left|k_{1}-k_{2}\right| \leqslant 5$, and $k_{3}, k_{4} \leqslant k_{1}-10$, then we have

$$
\left\|\mathbf{P}_{k_{4}} \mathcal{N}\left(u_{1}, u_{2}, u_{3}\right)\right\|_{N_{M, k_{4}}^{\alpha}} \lesssim \min \left(\Lambda_{3}, \Lambda_{4}\right)\left\|u_{1}\right\|_{F_{M, k_{1}}^{\alpha}}\left\|u_{2}\right\|_{F_{M, k_{2}}^{\alpha}}\left\|u_{3}\right\|_{F_{M, k_{3}}^{\alpha}},
$$

where $\Lambda_{3}$ and $\Lambda_{4}$ are given by

$$
\Lambda_{3}=2^{(-3 / 2+\alpha+\varepsilon) k_{1}^{*}-\alpha k_{4}-\beta} \quad \text { with } \beta= \begin{cases}k_{3}^{*} / 2 & \text { if }\left|k_{3}-k_{4}\right| \geqslant 2, \\ 0 & \text { otherwise, }\end{cases}
$$

and

$$
\Lambda_{4}= \begin{cases}2^{-(1 / 2)(3-\alpha-\varepsilon) k_{1}^{*}-k_{3}^{*} / 2+k_{4}^{*} / 2-\alpha k_{4}} & \text { if }\left|k_{3}-k_{4}\right| \geqslant 2, \\ 2^{-(1 / 2)(3-\alpha-\varepsilon) k_{1}^{*}+k_{3}^{*} / 4-\alpha k_{4}} & \text { otherwise, }\end{cases}
$$

for any $\varepsilon>0$.

Proof. We proceed as in the proof of Lemma 4.4, Then, (4.9) with $\Lambda_{3}$ follows from (3.4) in Lemma 3.1 with the size estimates (4.1) and (4.2). Similarly, (4.9) with $\Lambda_{4}$ follows from Lemma 3.5.

In the following, we briefly discuss the proof of Proposition 4.1. From Lemmas 4.2-4.5, we have

$$
\begin{gathered}
2^{s k_{4}}\left\{\left\|\mathbf{P}_{k_{4}} \mathcal{N}\left(u_{1}, u_{2}, u_{3}\right)\right\|_{N_{M, k_{4}}^{\alpha}}+\left\|\mathbf{P}_{k_{4}} \mathcal{R}\left(u_{1}, u_{2}, u_{3}\right)\right\|_{N_{M, k_{4}}^{\alpha}}\right\} \\
\lesssim 2^{s k_{4}} \Lambda^{*}\left\|u_{1}\right\|_{F_{M, k_{1}}^{\alpha}}^{\alpha}\left\|u_{2}\right\|_{F_{M, k_{2}}^{\alpha}}\left\|u_{3}\right\|_{F_{M, k_{3}}^{\alpha}},
\end{gathered}
$$

where $\Lambda^{*}$ denotes the constants in Lemmas 4.2-4.5, depending on different frequency interactions. Note that it suffices to guarantee that

$$
2^{s k_{4}} \Lambda^{*} \lesssim 2^{-\varepsilon k_{1}^{*}} 2^{s\left(k_{1}+k_{2}+k_{3}\right)} .
$$

Then, Proposition 4.1 follows from summing (4.10) over different dyadic blocks. Moreover, at a slight expense of the regularity in modulation, we can gain a factor $T^{\theta}$ for some $\theta>0$. See [19] for the details.

In the following, we perform case-by-case analysis on the constants obtained in Lemmas 4.2-4.5 and compute the restrictions on $s<0$ and $\alpha>0$ such that (4.11) holds. In the following, $\varepsilon=\varepsilon(s)>0$ denotes a small constant which may vary line by line.

(i) The output frequency is high. In view of Lemma 4.2, we need to have $-\left(\frac{3}{4} \alpha-\varepsilon\right) \leqslant 2 s$. Hence, it suffices to choose

$$
\alpha=-\frac{8 s}{3}+\varepsilon
$$


for some sufficiently small $\varepsilon=\varepsilon(s)>0$. Note that this is consistent with the heuristics presented in Section 2.4.

(ii) High $\times$ high $\times$ high $\rightarrow$ low: In view of (4.8) with $\Lambda_{1}$ of Lemma 4.4, we need to have

$$
-2+\alpha+\varepsilon \leqslant 3 s
$$

Then, it follows from (4.12) that (4.13) holds for $s \geqslant-\frac{6}{17}+\varepsilon$. Next, we consider the case $s \leqslant-\frac{6}{17}$. Then, from (4.8) with $\Lambda_{2}$, we need to have

$$
\left(-2+\frac{\alpha}{2}+\varepsilon\right) k_{1}^{*}+\left(s+\frac{1}{2}-\alpha\right) k_{4}^{*} \leqslant 3 s k_{1}^{*} .
$$

In view of (4.12), we must have $s \geqslant-\frac{6}{13}+\varepsilon$ from the coefficients of $k_{1}^{*}$, while we have $s \leqslant-\frac{3}{22}+\varepsilon$ from the coefficient of $k_{4}^{*}$. Hence, it follows from (4.13) and (4.14) that (4.11) holds for any $s \in\left(-\frac{6}{13}, 0\right)$.

(iii) High $\times$ high $\times$ low $\rightarrow$ low: First, we consider $s>-\frac{9}{28}$. From (4.9) with $\Lambda_{3}$ of Lemma 4.5, we need to have

$$
\left(-\frac{3}{2}+\alpha+\varepsilon\right) k_{1}^{*} \leqslant 2 s k_{1}^{*} \quad \text { and } \quad(s-\alpha) k_{4}-\beta \leqslant s k_{3} .
$$

In view of (4.12), the first condition provides $s \geqslant-\frac{9}{28}+\varepsilon$. The second condition is trivially satisfied when $k_{4} \geqslant k_{3}-5$. When $k_{3} \geqslant k_{4}+5$, it gives $s \geqslant-\frac{1}{2}$. Hence, (4.11) holds for $s \in\left(-\frac{9}{28}, 0\right)$.

Next, we consider $s \leqslant-\frac{9}{28}$. First, we consider the case $\left|k_{3}-k_{4}\right| \leqslant 1$. From (4.9) with $\Lambda_{4}$, we need to have

$$
-\frac{1}{2}(3-\alpha-\varepsilon) k_{1}^{*} \leqslant 2 s k_{1}^{*} \quad \text { and } \quad\left(\frac{1}{4}-\alpha\right) k_{3}^{*} \leqslant 0 .
$$

In view of (4.12), the first condition provides $s \geqslant-\frac{9}{20}+\varepsilon$, while the second condition provides $s \leqslant-\frac{3}{32}+\varepsilon$.

Next, let us consider the case $k_{3} \geqslant k_{4}+2$. (The case $k_{4} \geqslant k_{3}+2$ is easier.) In this case, we need to have

$$
-\frac{1}{2}(3-\alpha-\varepsilon) k_{1}^{*}-\frac{1}{2} k_{3}+\left(s+\frac{1}{2}-\alpha\right) k_{4} \leqslant 2 s k_{1}^{*}+s k_{3} .
$$

This yields the condition $s \in\left(-\frac{9}{20},-\frac{27}{220}\right)$. Hence, it follows from (4.15) and (4.16) that (4.11) holds for any $s \in\left(-\frac{9}{20},-\frac{9}{28}\right]$.

Putting all the cases (i)-(iii) together, we see that (4.11) holds for $s \in\left(-\frac{9}{20}, 0\right)$. 


\section{Energy estimate on smooth solutions}

In this section, we establish an energy estimate for (smooth) solutions to the Wick ordered cubic 4NLS (1.5). Let $u \in C\left(\mathbb{R} ; H^{\infty}(\mathbb{T})\right)$ be a smooth solution to (1.5). Then, in view of (2.18), our goal is to estimate

$$
\sup _{t \in[-T, T]} E_{k_{0}}(u)(t)
$$

in a summable manner over $k_{0} \in \mathbb{Z}_{M}$, where $E_{k_{0}}(u)$ is as in (2.16). By the fundamental theorem of calculus with the equation (1.5), we have

$$
\begin{aligned}
E_{k_{0}}(u)(t)-E_{k_{0}}(u)(0)= & 2 \operatorname{Re}\left(\int_{0}^{t} \sum_{n \in \mathbb{Z}} a_{k_{0}}(n) \partial_{t} \widehat{u}_{n}\left(t^{\prime}\right) \widehat{\widehat{u}}_{n}\left(t^{\prime}\right) d t^{\prime}\right) \\
= & -2 \operatorname{Re} i\left(\int_{0}^{t} \sum_{n \in \mathbb{Z}} a_{k_{0}}(n) \sum_{\Gamma(n)} \widehat{u}_{n_{1}}{\widehat{\widehat{u}_{n}}}_{n_{2}} \widehat{u}_{n_{3}} \widehat{\widehat{u}}_{n}\left(t^{\prime}\right) d t^{\prime}\right) \\
& +\underbrace{2 \operatorname{Re} i\left(\int_{0}^{t} \sum_{n \in \mathbb{Z}} a_{k_{0}}(n)\left|\widehat{u}_{n}\left(t^{\prime}\right)\right|^{4} d t^{\prime}\right)}_{=0},
\end{aligned}
$$

where $\Gamma(n)$ is as in (2.3). By letting $n_{4}=n$ and symmetrizing under the summation indices $n_{1}, \ldots, n_{4}$, we obtain

$$
\begin{aligned}
E_{k_{0}}(u)(t)-E_{k_{0}}(u)(0) & =\frac{i}{2} \int_{0}^{t} \sum_{\substack{n_{1}-n_{2}+n_{3}-n_{4}=0 \\
n_{2} \neq n_{1}, n_{3}}} \Psi(\bar{n}) \widehat{u}_{n_{1}} \widehat{\widehat{u}}_{n_{2}} \widehat{u}_{n_{3}}{\overline{\widehat{u}_{n}}}_{n_{4}}\left(t^{\prime}\right) d t^{\prime} \\
& =: R_{k_{0}}(t),
\end{aligned}
$$

where $\Psi(\bar{n})$ is defined by

$$
\Psi(\bar{n})=a_{k_{0}}\left(n_{1}\right)-a_{k_{0}}\left(n_{2}\right)+a_{k_{0}}\left(n_{3}\right)-a_{k_{0}}\left(n_{4}\right) .
$$

The symbol $\Psi(\bar{n})$ provides an extra decay via the mean value theorem and the double mean value theorem [13, Lemmas 4.1 and 4.2] applied to the symbol $a_{k_{0}}(\xi)$. See (5.8), (5.9), and (5.10).

REMARK 5.1. In this section, we study an energy estimate on a single solution. In Section 7, we establish an energy estimate for the difference of two solutions in order to prove uniqueness of solutions. It is significantly harder to establish an energy estimate for the difference of two solutions mainly due to (i) the resonant contribution for the difference of solutions (corresponding the second term on 
the right-hand side of (5.1)) does not vanish and (ii) the symmetrization process above fails for the difference of solutions. In order to overcome this difficulty, we perform an infinite iteration of normal form reductions.

The main goal of this section is to establish the following multilinear estimate on $R_{k_{0}}$.

Proposition 5.2. Let $s \in\left(-\frac{9}{20}, 0\right)$ and $\alpha=-8 s / 3+$ as in (4.12). Then, there exist $\delta_{0}>0$ and $\theta>0$ such that

$$
\left|R_{k_{0}}(T)\right| \lesssim 2^{-\delta_{0} k_{0}} T^{\theta}\|u\|_{F^{s, \alpha}(T)}^{4}
$$

for all $k_{0} \in \mathbb{Z}_{M}$ and $0<T \leqslant 1$.

In [19], we studied a similar energy estimate for solutions to the Wick ordered cubic NLS (1.12). There, we needed to perform a normal form reduction (that is, add a correction term) in order to achieve a better energy estimate and hence match the regularity from the trilinear estimate. The Wick ordered cubic 4NLS (1.5), however, possesses much stronger dispersion and we do not perform a normal form reduction.

REMARK 5.3. As in [19], the energy estimate (5.4) possesses a certain smoothing property, namely, (5.4) still holds true even if we replace the $F^{s, \alpha}(T)$-norm on the right-hand side by $F^{s-\delta, \alpha}(T)$-norm for some small $\delta>0$. This smoothing property plays an important role in proving a compactness property of smooth approximating solutions (Lemma 6.3; see also Lemma 7.4). See the proof of Lemma 8.2 in [19].

Proof of Proposition 5.2. We first write $R_{k_{0}}$ as a multilinear operator given by

$$
\begin{aligned}
R_{k_{0}}(t) & =R_{k_{0}}\left(u_{1}, u_{2}, u_{3}, u_{4}\right)(t) \\
& =\frac{i}{2} \int_{0}^{t} \sum_{\substack{n_{1}-n_{2}+n_{3}-n_{4}=0 \\
n_{2} \neq n_{1}, n_{3}}} \Psi(\bar{n}) \widehat{u}_{1}\left(n_{1}\right) \overline{\widehat{u}_{2}\left(n_{2}\right)} \widehat{u}_{3}\left(n_{3}\right) \overline{\widehat{u}_{4}\left(n_{4}\right)}\left(t^{\prime}\right) d t^{\prime} .
\end{aligned}
$$

Apply the dyadic decomposition on the spatial frequencies $\left|n_{i}\right| \sim 2^{k_{i}}, k_{i} \geqslant \log _{2} M$, $i=1, \ldots, 4$. By symmetry, assume that $\left|n_{1}\right| \sim n_{1}^{*}$. Then, it suffices to prove 


$$
\left|R_{k_{0}}(T)\right| \lesssim T^{\theta} 2^{-\delta_{0} k_{0}} \prod_{i=1}^{4} 2^{(s-) k_{i}}\left\|\mathbf{P}_{k_{i}} u_{i}\right\|_{F_{M, k_{i}}^{\alpha}(T)}
$$

Here, a small extra decay is needed to sum over dyadic blocks. Let $\tilde{u}_{i}$ be an extension of $u_{i}$ such that $\left\|\tilde{u}_{i}\right\|_{F_{M, k_{i}}^{\alpha}} \leqslant 2\left\|\mathbf{P}_{k_{i}} u\right\|_{F_{M, k_{i}}^{\alpha}(T)}$. Let $\gamma: \mathbb{R} \rightarrow[0,1]$ be a smooth cutoff function supported on $[-1,1]$ such that

$$
\sum_{m \in \mathbb{Z}} \gamma^{4}(t-m) \equiv 1, \quad t \in \mathbb{R}
$$

With $K=k_{1}^{*}+c$, define $f_{i, j_{i}, m}, j_{i} \in \mathbb{Z}_{\geqslant 0}, i=1, \ldots, 4$, by

$$
f_{i, j_{i}, m}=\mathcal{F}^{-1}\left[\eta_{j_{i}}\left(\tau+n^{4}\right) \mathcal{F}\left[\gamma\left(2^{[\alpha K]} t-m\right) \tilde{u}_{i}\right]\right] \text {. }
$$

Then, it suffices to prove

$$
\begin{aligned}
\mid \int_{\mathbb{R}} \mathbf{1}_{[0, T]}(t) & \sum_{j_{1}, \ldots, j_{4} \in \mathbb{Z}_{\geqslant 0}|m| \leqslant 2^{[\alpha K]}(T+1)} \sum_{\substack{n_{1}-n_{2}+n_{3}-n_{4}=0 \\
n_{2} \neq n_{1}, n_{3}}} \Psi(\bar{n}){\widehat{f_{1}}}_{1, j_{1}, m}\left(n_{1}\right) \overline{\widehat{f}_{2, j_{2}, m}\left(n_{2}\right)} \\
& \times \widehat{f}_{3, j_{3}, m}\left(n_{3}\right) \widehat{\widehat{f}_{4, j_{4}, m}\left(n_{4}\right)}(t) d t \mid \lesssim T^{\theta} 2^{-\delta_{0} k_{0}} \prod_{i=1}^{4} 2^{(s-) k_{i}}\left\|\widetilde{u}_{i}\right\|_{F_{M, k_{i}}^{\alpha}} .
\end{aligned}
$$

In the following, we prove (5.5) for each dyadic modulation size $\sim 2^{j_{i}}, i=1$, $\ldots, 4$. In view of (2.8), we assume that $j_{i} \geqslant \alpha K$. Define the subsets $\mathcal{A}$ and $\mathcal{B}$ of $\left\{m \in \mathbb{Z}:|m| \leqslant 2^{[\alpha K]}(T+1)\right\}$ by

$$
\begin{gathered}
\mathcal{A}=\left\{m \in \mathbb{Z}: \mathbf{1}_{[0, T]}(t) \gamma\left(2^{[\alpha K]} t-m\right)=\gamma\left(2^{[\alpha K]} t-m\right)\right\}, \\
\mathcal{B}=\left\{m \in \mathbb{Z}: \mathbf{1}_{[0, T]}(t) \gamma\left(2^{[\alpha K]} t-m\right) \neq \gamma\left(2^{[\alpha K]} t-m\right)\right. \\
\text { and } \left.\mathbf{1}_{[0, T]}(t) \gamma\left(2^{[\alpha K]} t-m\right) \neq 0\right\} .
\end{gathered}
$$

Namely, $\mathcal{A}$ denotes the set of $m \in \mathbb{Z}$ such that the support of $\gamma\left(2^{[\alpha K]} t-m\right)$ lies in the interior of the interval $[0, T]$, while $\mathcal{B}$ denotes those $m \in \mathbb{Z}$ such that the support of $\gamma\left(2^{[\alpha K]} t-m\right)$ intersects the boundary point $t=0$ or $t=T$. In the following, we separately estimate the contributions from $\mathcal{A}$ and $\mathcal{B}$. Finally, we simply denote $f_{i, j_{i}, m}$ by $f_{i, j_{i}}$ in the following.

Part 1: First, we consider the terms with $m \in \mathcal{A}$. Note that we can drop the sharp cutoff $\mathbf{1}_{[0, T]}(t)$ on the left-hand side of (5.5) in this case. We prove (5.5) with $\theta=1$ in this case. The main ingredients are the (double) mean value theorem and the following lower bound on the largest modulation; with $\sigma_{j}=\tau_{j}+n_{j}^{4}$, we have

$$
\sigma_{1}^{*}:=\max \left(\left|\sigma_{1}\right|,\left|\sigma_{2}\right|,\left|\sigma_{3}\right|,\left|\sigma_{4}\right|\right) \gtrsim|\phi(\bar{n})| \sim\left(n_{1}^{*}\right)^{2}|\mu(\bar{n})|,
$$


where $\phi(\bar{n})$ and $\mu(\bar{n})$ are as in (2.4) and (2.5). Recall that we have $2^{k_{i}} \geqslant M$, $i=1, \ldots, 4$. Given $k \in \mathbb{Z}_{M}$, it follows from (2.12) and (2.13) that

$$
\left|\partial^{\gamma} a_{k_{0}}(\xi)\right| \lesssim 2^{(2 s-\gamma) k} 2^{-\delta_{0}\left|k-k_{0}\right|}
$$

for $\xi \in I_{k}^{M}, \gamma=1,2$.

- Case (a): $2^{k_{1}^{*}} \lesssim M$.

In this case, we have $2^{k_{1}^{*}} \sim 2^{k_{4}^{*}}$. Then, by the double mean value theorem [13, Lemma 4.2], we have

$$
\begin{aligned}
|\Psi(\bar{n})| & \lesssim\left|a_{k_{0}}^{\prime \prime}\left(n_{1}^{*}\right)\right| \cdot\left|\left(n_{4}-n_{1}\right)\left(n_{4}-n_{3}\right)\right| \lesssim 2^{(2 s-2) k_{1}^{*}-\delta_{0}\left|k_{1}^{*}-k_{0}\right|}|\mu(\bar{n})| \\
& \lesssim 2^{-\delta_{0} k_{0}} 2^{\left(2 s-2+\delta_{0}\right) k_{1}^{*}}|\mu(\bar{n})| .
\end{aligned}
$$

We first consider the case $n_{1}^{*} \gtrsim 2^{k_{1}^{*}}$. In this case, we apply Lemma 3.3 in view of Remark 3.4. With (4.12), we have

$$
\begin{aligned}
& \sum_{|m| \leqslant 2^{[\alpha K]}(T+1)}\left(\sigma_{1}^{*}\right)^{-1 / 2}|\Psi(\bar{n})| 2^{(-\alpha / 2+1 / 2) k_{1}^{*}} \lesssim T 2^{-\delta_{0} k_{0}} 2^{\alpha k_{1}^{*} / 2}|\mu(\bar{n})|^{1 / 2} 2^{\left(2 s-5 / 2+\delta_{0}\right) k_{1}^{*}} \\
& \lesssim T 2^{-\delta_{0} k_{0}} 2^{\left((2 / 3) s-3 / 2+\delta_{0}+\right) k_{1}^{*}} \leqslant T 2^{-\delta_{0} k_{0}} 2^{(4 s-) k_{1}^{*}}
\end{aligned}
$$

for sufficiently small $\delta_{0}=\delta_{0}(s)>0$, provided that $s>-\frac{9}{20}$. Then, (5.5) follows from Lemma 3.3 with (5.7) in this case.

Next, we consider the case $n_{1}^{*} \ll 2^{k_{1}^{*}}$. In this case, Lemma 3.3 is not applicable. Note, however, that this case occurs only when $n_{1}^{*} \ll M$. Moreover, from the definition of the symbol $a_{k_{0}}$, we see that $a_{k_{0}}(n)$ is constant for $|n| \leqslant M / 2$. Hence, we conclude that $\Psi(\bar{n})=0$ when $n_{1}^{*} \ll M$ and there is no contribution to (5.5) in this case.

- Case (b): $\left|n_{4}-n_{1}\right|,\left|n_{4}-n_{3}\right| \ll n_{1}^{*}$ and $2^{k_{1}^{*}} \gg M$.

In this case, we have $\left|n_{1}\right| \sim\left|n_{2}\right| \sim\left|n_{3}\right| \sim\left|n_{4}\right| \sim n_{1}^{*} \sim 2^{k_{1}^{*}}$. Moreover, by the double mean value theorem, we have

$$
|\Psi(\bar{n})| \lesssim 2^{\left(2 s-2+\delta_{0}\right) k_{1}^{*}-\delta_{0} k_{0}}|\mu(\bar{n})|
$$

as in Case (a). Then, the rest follows as in Case (a).

- Case (c): $\left|n_{4}-n_{1}\right| \sim n_{1}^{*} \gg\left|n_{4}-n_{3}\right|=\left|n_{1}-n_{2}\right|$ and $2^{k_{1}^{*}} \gg M$.

In this case, we have $\left|n_{2}\right| \sim\left|n_{1}\right| \sim n_{1}^{*}$. Then, by the mean value theorem, we have

$$
\left|a_{k_{0}}\left(n_{1}\right)-a_{k_{0}}\left(n_{2}\right)\right| \lesssim\left|a_{k_{0}}^{\prime}\left(n_{1}^{*}\right)\right| \cdot\left|n_{1}-n_{2}\right| \lesssim 2^{-\delta_{0} k_{0}} 2^{\left(2 s-1+\delta_{0}\right) k_{1}^{*}}\left|n_{1}-n_{2}\right| .
$$

Moreover, from (2.4), we have $|\phi(\bar{n})| \sim\left(n_{1}^{*}\right)^{3}\left|n_{4}-n_{3}\right|$. 
○ Subcase (c.i): $\left|n_{4}-n_{3}\right| \ll n_{3}^{*}$.

In this case, we also have $\left|n_{3}\right| \sim\left|n_{4}\right| \sim n_{3}^{*}$. Then, by the mean value theorem, we have

$$
\left|a_{k_{0}}\left(n_{3}\right)-a_{k_{0}}\left(n_{4}\right)\right| \lesssim\left|a_{k_{0}}^{\prime}\left(n_{3}^{*}\right)\right| \cdot\left|n_{4}-n_{3}\right| \lesssim 2^{-\delta_{0} k_{0}} 2^{\left(2 s-1+\delta_{0}\right) k_{3}^{*}}\left|n_{4}-n_{3}\right| .
$$

We point out that (5.10) holds true even when $n_{3}^{*} \lesssim M$, since $a_{k_{0}}^{\prime}(n)=0$ for $|n| \leqslant M / 2$. See (2.14). Hence, it follows from (5.9) and (5.10) that

$$
|\Psi(\bar{n})| \lesssim 2^{-\delta_{0} k_{0}} 2^{\left(2 s-1+\delta_{0}\right) k_{3}^{*}}\left|n_{4}-n_{3}\right|
$$

and thus

$$
\begin{aligned}
& \sum_{|m| \leqslant 2^{[\alpha K]}(T+1)}\left(\sigma_{1}^{*}\right)^{-1 / 2}|\Psi(\bar{n})| 2^{-(\alpha / 2) k_{1}^{*}} 2^{k_{4}^{*} / 2} \\
& \quad \lesssim T 2^{-\delta_{0} k_{0}} 2^{(-(4 / 3) s-3 / 2+) k_{1}^{*}} 2^{\left(2 s-1 / 2+\delta_{0}\right) k_{3}^{*}}\left|n_{4}-n_{3}\right|^{1 / 2} \\
& \quad \lesssim T 2^{-\delta_{0} k_{0}} 2^{\left(-(4 / 3) s-3 / 2+\delta_{0}+\right) k_{1}^{*}} 2^{2 s k_{3}^{*}} \lesssim T 2^{-\delta_{0} k_{0}} 2^{\left(-(10 / 3) s-3 / 2+\delta_{0}+\right) k_{1}^{*}}\left(\prod_{i=1}^{4} 2^{(s-) k_{i}}\right) .
\end{aligned}
$$

Hence, (5.5) follows from Lemma 3.3, provided that $s>-\frac{9}{20}$ and $\delta_{0}=\delta_{0}(s)>0$ is sufficiently small.

o Subcase (c.ii): $\left|n_{4}-n_{3}\right| \sim n_{3}^{*}$.

In this case, we have $|\phi(\bar{n})| \gtrsim\left(n_{1}^{*}\right)^{3} n_{3}^{*}$ and $|\Psi(\bar{n})| \lesssim 2^{-\delta_{0} k_{0}} 2^{\left(2 s+\delta_{0}\right) k_{4}^{*}}$. Thus, we have

$$
\begin{aligned}
& \sum_{|m| \leqslant 2^{[\alpha K]}(T+1)}\left(\sigma_{1}^{*}\right)^{-1 / 2}|\Psi(\bar{n})| 2^{-(\alpha / 2) k_{1}^{*}} 2^{k_{4}^{*} / 2} \\
& \lesssim T 2^{-\delta_{0} k_{0}} 2^{\left(-(10 / 3) s-3 / 2+\delta_{0}+\right) k_{1}^{*}} 2^{(-s-1 / 2) k_{3}^{*}} 2^{(s+1 / 2) k_{4}^{*}}\left(\prod_{i=1}^{4} 2^{(s-) k_{i}}\right) .
\end{aligned}
$$

Hence, (5.5) follows from Lemma 3.3, provided that $s>-\frac{9}{20}$ and $\delta_{0}>0$ is sufficiently small.

- Case (d): $\left|n_{4}-n_{1}\right|,\left|n_{4}-n_{3}\right| \sim n_{1}^{*}$ and $2^{k_{1}^{*}} \gg M$.

In this case, we have $|\phi(\bar{n})| \sim\left(n_{1}^{*}\right)^{4}$ and $|\Psi(\bar{n})| \lesssim 2^{-\delta_{0} k_{0}} 2^{\left(2 s+\delta_{0}\right) k_{4}^{*}}$. Thus, we have

$$
\begin{aligned}
& \sum_{|m| \leqslant 2^{[\alpha K]}(T+1)}\left(\sigma_{1}^{*}\right)^{-1 / 2}|\Psi(\bar{n})| 2^{-(\alpha / 2) k_{1}^{*}} 2^{k_{4}^{*} / 2} \\
& \lesssim T 2^{-\delta_{0} k_{0}} 2^{\left(-(13 / 3) s-2+\delta_{0}+\right) k_{1}^{*}} 2^{(s+1 / 2) k_{4}^{*}}\left(\prod_{i=1}^{4} 2^{(s-) k_{i}}\right) .
\end{aligned}
$$


Hence, (5.5) follows from Lemma 3.3, provided that $s>-\frac{9}{20}$ and $\delta_{0}>0$ is sufficiently small.

Part 2: Next, we consider the terms with $m \in \mathcal{B}$. In this case, we use Lemma 2.4 to handle the sharp cutoff $\mathbf{1}_{[0, T]}$. Note that there are only $O(1)$ many values of $m \in \mathcal{B}$. Namely, we can save $\left(n_{1}^{*}\right)^{-\alpha}$ as compared to the analysis in Part 1.

We only consider Case (a) above as the other cases follow in a similar manner. With (5.6) and $|\phi(\bar{n})| \sim\left(n_{1}^{*}\right)^{2}|\mu(\bar{n})|$, we have

$$
\left(\sigma_{1}^{*}\right)^{-1 / 2+\theta+}|\Psi(\bar{n})| \lesssim 2^{-\delta_{0} k_{0}}\left(n_{1}^{*}\right)^{4 s-}
$$

for $\theta>0$ sufficiently small such that $-1+2 \theta<s$. Suppose $\sigma_{1}=\sigma_{1}^{*}$. Then, by $L_{x, t}^{2}, L_{x, t}^{6}, L_{x, t}^{6}, L_{x, t}^{6}$-Hölder's inequality and Lemma 2.4, we have

$$
\begin{aligned}
\left|R_{k_{0}}(T)\right| & \lesssim 2^{-\delta_{0} k_{0}}\left(n_{1}^{*}\right)^{4 s-} \sup _{j_{1}} 2^{(1 / 2-\theta-) j_{1}}\left\|\mathcal{F}\left(\mathbf{1}_{[0, T]} f_{1, j_{1}}\right)\right\|_{\ell_{n}^{2} L_{t}^{2}} \sum_{j_{2}, j_{3}, j_{4}} \prod_{i=2}^{4}\left\|f_{i, j_{i}}\right\|_{L_{x, t}^{6}} \\
& \lesssim T^{\theta} 2^{-\delta_{0} k_{0}} \prod_{i=1}^{4} 2^{(s-) k_{i}}\left\|\mathbf{P}_{k_{i}} \widetilde{u}_{i}\right\|_{F_{M, k_{i}}^{\alpha}},
\end{aligned}
$$

where we used Lemma 2.3 in the last step. This completes the proof of Proposition 5.2.

\section{Global existence}

In this section, we prove global existence (Theorem 1.1) by putting together the trilinear estimate (Proposition 4.1) and the energy estimate (Proposition 5.2). We also make use of the decay property (2.6) of the $H_{M}^{s}$-norm as $M \rightarrow \infty$. Moreover, we establish an exponential growth bound on the $H^{s}$-norms of solutions. In view of the time reversibility of (1.5), we only consider positive times in the following.

6.1. Proof of Theorem 1.1. The following proposition establishes the longtime existence for small initial data which plays a key role in the proof of Theorem 1.1.

Proposition 6.1. Let $s \in\left(-\frac{9}{20}, 0\right)$. Then, given $u_{0} \in H^{s}(\mathbb{T})$, there exist $T=$ $T\left(\left\|u_{0}\right\|_{H^{s}}\right)>0$ and a local-in-time solution $u$ to the Wick ordered cubic $4 N L S$ (1.5) on $[0, T]$ with $\left.u\right|_{t=0}=u_{0}$. Furthermore, there exists $\varepsilon_{0}>0$ such that if $u_{0} \in H^{s}(\mathbb{T})$ satisfies

$$
\left\|u_{0}\right\|_{H_{M}^{s}} \leqslant \varepsilon_{0}
$$


for some dyadic $M \geqslant 1$, then the corresponding solution $u$ to (1.5) with $\left.u\right|_{t=0}=u_{0}$ can be extended to the unit time interval $[0,1]$ with the following estimate:

$$
\sup _{t \in[0,1]}\|u(t)\|_{H_{M}^{s}} \leqslant 2\left\|u_{0}\right\|_{H_{M}^{s}} .
$$

We first assume Proposition 6.1 and present the proof of Theorem 1.1.

Proof of Theorem 1.1. Given $T>0$, we iteratively apply Proposition 6.1 and construct a solution $u$ on $[0, T]$. Let $u_{0} \in H^{s}(\mathbb{T})$. Then, there exists $M=M(s$, $\left.T, u_{0}, \varepsilon_{0}\right) \geqslant 1$ such that

$$
\left\|u_{0}\right\|_{H_{M}^{s}} \leqslant 2^{-[T]-1} \varepsilon_{0}
$$

where $\varepsilon_{0}$ is as in Proposition 6.1. Hence, we can apply Proposition $6.1[T]+1$ times and construct the solution $u$ on $[0, T]$, satisfying

$$
\sup _{t \in[0, T]}\|u(t)\|_{H_{M}^{s}} \leqslant 2^{[T]+1}\left\|u_{0}\right\|_{H_{M}^{s}} \leqslant \varepsilon_{0}
$$

This proves Theorem 1.1.

Before proceeding to the proof of Proposition 6.1, we recall the following lemma [19, Lemma 8.1].

LemMA 6.2. Let $s \in \mathbb{R}$. Given $u \in C\left(\mathbb{R} ; H^{\infty}(\mathbb{T})\right)$, let $X_{M}(T)=\|u\|_{E_{M}^{s}(T)}+$ $\|\mathfrak{N}(u)\|_{N_{M}^{s, \alpha}(T)}$. Then, $X_{M}(T)$ is nondecreasing and continuous in $T \in \mathbb{R}_{+}$. Moreover, we have

$$
\lim _{T \rightarrow 0} X_{M}(T)=\|u(0)\|_{H_{M}^{s}} .
$$

While our function spaces depend on the parameter $M \geqslant 1$, the proof of Lemma 8.1 in [19] applies to Lemma 6.2 without any change for fixed $M \geqslant 1$.

Proof of Proposition 6.1. We only sketch the proof under the smallness assumption (6.1), since it follows closely the argument in [19, Section 8]. See also Remark 6.4. Fix $s \in\left(-\frac{9}{20}, 0\right)$ and $\alpha=-\frac{8}{3} s+$. Let $u \in C\left(\mathbb{R} ; H^{\infty}(\mathbb{T})\right)$ be a smooth solution to (1.5) with $\left.u\right|_{t=0}=u_{0}$. Then, it follows from Lemma 2.5, Proposition 4.1, (2.18), and Proposition 5.2 that there exists $\theta=\theta(s)>0$ such that

$$
\begin{aligned}
\|u\|_{F_{M}^{s, \alpha}(T)} & \lesssim\|u\|_{E_{M}^{s}(T)}+\|\mathfrak{N}(u)\|_{N_{M}^{s, \alpha}(T)}, \\
\|\mathfrak{N}(u)\|_{N_{M}^{s, \alpha}(T)} & \lesssim T^{\theta}\|u\|_{F_{M}^{s, \alpha}(T)}^{3}, \\
\|u\|_{E_{M}^{s}(T)}^{2} & \leqslant\left\|u_{0}\right\|_{H_{M}^{s}}^{2}+C T^{\theta}\|u\|_{F_{M}^{s, \alpha}(T)}^{4},
\end{aligned}
$$


for any $T>0$ and $M \geqslant 1$, where $\mathfrak{N}(u)=\mathcal{N}(u)+\mathcal{R}(u)$ denotes the nonlinearity of (1.5) defined in (1.6). Letting $X_{M}(T)$ be as in Lemma 6.2, it follows from (6.3), (6.4), and (6.5) that

$$
X_{M}(T)^{2} \leqslant 2\left\|u_{0}\right\|_{H_{M}^{s}}^{2}+C T^{\theta}\left\{X_{M}(T)^{2}+X_{M}(T)^{4}\right\} \cdot X_{M}(T)^{2}
$$

for any $T>0$. Now, choose $\varepsilon_{0}>0$ sufficiently small such that

$$
C\left(4 \varepsilon_{0}^{2}+16 \varepsilon_{0}^{4}\right) \leqslant \frac{1}{2} .
$$

Then, in view of Lemma 6.2, it follows from a continuity argument that

$$
X_{M}(T) \leqslant 2\left\|u_{0}\right\|_{H_{M}^{s}}
$$

for any $T \in(0,1]$. Hence, the a priori bound (6.2) for smooth solutions follows from (6.6) and (2.10).

Next, we recall the following compactness lemma.

LEMMA 6.3. Let $s>-\frac{9}{20}$. Given $u_{0} \in H_{M}^{s}(\mathbb{T})$, let $u_{n} \in C\left(\mathbb{R} ; H^{\infty}(\mathbb{T})\right)$ be a global solution to (1.5) with $\left.u_{n}\right|_{t=0}=\mathbf{P}_{\leqslant n} u_{0}$. Then, there exists $T_{0}=T_{0}\left(\left\|u_{0}\right\|_{H^{s}}\right)>0$ such that the set $\left\{u_{n}\right\}_{n \in \mathbb{N}}$ is precompact in $C\left([-T, T] ; H_{M}^{s}(\mathbb{T})\right)$ for $T \leqslant T_{0}$. Moreover, $\left\|\mathbf{P}_{>N} u_{n}\right\|_{C_{T} H_{M}^{s}}$ tends to 0 as $N \rightarrow \infty$, uniformly in $n \in \mathbb{N}$.

See [19, Lemma 8.2] for the details of the proof. See also Lemma 7.4 below. We point out that the smoothing property of the energy estimate in Proposition 5.2 plays an important role in proving Lemma 6.3.

In view of Lemma 6.3 with $T_{0}=1$, we can extract a subsequence, which we still denote by $\left\{u_{n}\right\}_{n \in \mathbb{N}}$, converging to some $u$ in $C\left([0,1] ; H_{M}^{s}(\mathbb{T})\right)$. It remains to show that this limit $u$ is a distributional solution to (1.5). It follows from Lemma 6.3 that $\left\{u_{n}\right\}_{n \in \mathbb{N}}$ also converges in $E_{M}^{s}(1)$. In view of (6.3) and (6.4), this in turns implies that $\left\{u_{n}\right\}$ converges to $u$ in $F_{M}^{s, \alpha}(1)$. Finally, by applying the trilinear estimate (Proposition 4.1), we see that the nonlinearity $\left\{\mathfrak{N}\left(u_{n}\right)\right\}_{n \in \mathbb{N}}$ converges to $\mathfrak{N}(u)$ in $N_{M}^{s, \alpha}(1)$. Hence, the limit $u$ is a distributional solution to (1.5) on the time interval $[0,1]$. This proves local existence for the Wick ordered cubic 4NLS (1.5) in $H_{M}^{s}(\mathbb{T})$ for $s>-\frac{9}{20}$. Moreover, from the a priori estimate for smooth solutions and the convergence of $u_{n}$ to $u$ in $E_{M}^{s}(1),(6.2)$ also holds for the solution $u$.

REMARK 6.4. Let us briefly discuss the case when we do not impose the smallness assumption and $M=1$. This is the setting considered in [19] and hence is relevant for the proof of the nonexistence result (Corollary 1.2).

Let $R=\left\|u_{0}\right\|_{H^{s}}$ Then, choose $T_{0}=T_{0}(R) \leqslant 1$ sufficiently small such that

$$
C T_{0}^{\theta}\left(4 R^{2}+16 R^{4}\right) \leqslant \frac{1}{2} .
$$


Then, a continuity argument with Lemma 6.2 yields (6.6) for $T \in\left(0, T_{0}\right]$. By repeating the argument above, one can prove local existence on $\left[0, T_{0}\right]$ for $T_{0}=$ $T_{0}\left(\left\|u_{0}\right\|_{H^{s}}\right)>0$.

6.2. On the growth of Sobolev norms. In this subsection, we study the growth of the $H^{s}$-norm of a solution to (1.5), $s \in\left(-\frac{9}{20}, 0\right)$, constructed in Theorem 1.1.

Fix $s_{0} \in\left(-\frac{9}{20}, 0\right)$. The following bound follows from iterating Proposition 6.1.

Lemma 6.5. Let $s_{0} \leqslant s<0$ and $0<\varepsilon \leqslant \varepsilon_{0}$, where $\varepsilon_{0}$ is as in Proposition 6.1. Let $u$ be a solution to (1.5) with $\left.u\right|_{t=0}=u_{0} \in H^{s}(\mathbb{T})$ such that

$$
\left\|u_{0}\right\|_{H_{M}^{s_{0}}} \leqslant \varepsilon
$$

for some dyadic $M \geqslant 1$. Then, the following bound holds:

$$
\sup _{t \in[0, T]}\|u(t)\|_{H_{M}^{s}} \lesssim 2^{T}\left\|u_{0}\right\|_{H_{M}^{s}},
$$

for all $0<T \leqslant T_{0}$, where

$$
T_{0} \sim \log _{2}\left(\frac{\varepsilon_{0}}{\varepsilon}\right)
$$

Proof. When $s=s_{0}$, the estimate (6.7) follows from the proof of Proposition 6.1, namely iterating (6.2) $[T]+1$ times. For general $s \in\left(s_{0}, 0\right)$, we exploit the following equivalence

$$
\|f\|_{H_{M}^{s}}^{2} \sim \sum_{K \geqslant M} K^{-2 s_{0}+2 s}\|f\|_{H_{K}^{s_{0}}}^{2}
$$

for any $f \in H^{s}(\mathbb{T})$ and any dyadic $K \geqslant M \geqslant 1$. We first assume (6.8) and prove (6.7). By (6.8), (6.7) for $s=s_{0}$, and the monotonicity of the $H_{M}^{s}$-norm in $M$, we have

$$
\begin{aligned}
\sup _{t \in[0, T]}\|u(t)\|_{H_{M}^{s}}^{2} & \lesssim \sum_{K \geqslant M} K^{-2 s_{0}+2 s} \sup _{t \in[0, T]}\|u(t)\|_{H_{K}^{s_{0}}}^{2} \\
& \lesssim 2^{2 T} \sum_{K \geqslant M} K^{-2 s_{0}+2 s}\left\|u_{0}\right\|_{H_{K}^{s_{0}}}^{2} \sim 2^{2 T}\left\|u_{0}\right\|_{H_{M}^{s}}^{2} .
\end{aligned}
$$

This proves (6.7). 
It remains to show (6.8). Let us first consider the contribution from $|n| \leqslant M$. With $K^{2}+n^{2} \sim K^{2}$ for $K \geqslant M$, we have

$$
\sum_{K \geqslant M} K^{-2 s_{0}+2 s}\left\|f_{\leqslant M}\right\|_{H_{K}^{s_{0}}}^{2} \sim \sum_{K \geqslant M} K^{2 s}\left\|f_{\leqslant M}\right\|_{L^{2}}^{2} \sim M^{2 s}\left\|f_{\leqslant M}\right\|_{L^{2}}^{2} \sim\left\|f_{\leqslant M}\right\|_{H_{M}^{s}},
$$

where $f_{\leqslant M}=\mathcal{F}^{-1}\left[\mathbf{1}_{|n| \leqslant M} \widehat{f}\right]$. Next, we consider the contribution from $|n|>M$. By Fubini's theorem, we have

$$
\begin{aligned}
\sum_{K \geqslant M} K^{-2 s_{0}+2 s}\left\|f_{>M}\right\|_{H_{K}^{s_{0}}}^{2} & \sim \sum_{K \geqslant M} K^{2 s} \sum_{M<|n| \leqslant K}|\widehat{f}(n)|^{2} \\
& +\sum_{K \geqslant M} K^{-2 s_{0}+2 s} \sum_{|n|>K}|n|^{2 s_{0}}|\widehat{f}(n)|^{2} \\
& \sim \sum_{|n|>M}\left(\sum_{K \geqslant|n|} \frac{K^{2 s}}{|n|^{2 s}}\right)|n|^{2 s}|\widehat{f}(n)|^{2} \\
& +\sum_{|n|>M}\left(\sum_{M \leqslant K<|n|} \frac{K^{-2 s_{0}+2 s}}{|n|^{-2 s_{0}+2 s}}\right)|n|^{2 s}|\widehat{f}(n)|^{2} \\
& \sim\left\|f_{>M}\right\|_{H_{M}^{s}}^{2},
\end{aligned}
$$

where $f_{>M}=f-f_{\leqslant M}$. Then, (6.8) follows from (6.9) and (6.10).

By applying Lemma 6.5, we obtain the following global-in-time bound on the $H^{s}$-norm of solutions to (1.5) for $s_{0}<s<0$.

Proposition 6.6. Fix $s_{0} \in\left(-\frac{9}{20}, 0\right)$. Let $s \in\left(s_{0}, 0\right), B>0$, and $u$ be a solution to (1.5) with $\left.u\right|_{t=0}=u_{0} \in H^{s}(\mathbb{T})$ such that

$$
\left\|u_{0}\right\|_{H^{s}} \leqslant B
$$

Then, we have

$$
\|u(t)\|_{H^{s}} \lesssim \varepsilon_{0}^{s /\left(s-s_{0}\right)}\left(2^{t} B\right)^{1-s /\left(s-s_{0}\right)}
$$

for all $t>0$, where $\varepsilon_{0}$ is as in Proposition 6.1.

Proof. By choosing $M \gg 1$ sufficiently large, it follows from (6.8) that

$$
\left\|u_{0}\right\|_{H_{M}^{s_{0}}} \leqslant M^{-s+s_{0}} B \leqslant \varepsilon_{0} .
$$


Then, it follows from Lemma 6.5 that

$$
\sup _{t \in[0, T]}\|u(t)\|_{H_{M}^{s}} \lesssim 2^{T}\left\|u_{0}\right\|_{H_{M}^{s}}
$$

for all $T>0$ such that

$$
T \lesssim \log _{2}\left(\frac{M^{s-s_{0}} \varepsilon_{0}}{B}\right) . \quad \text { Namely, } M \gtrsim\left(\frac{2^{T} B}{\varepsilon_{0}}\right)^{1 /\left(s-s_{0}\right)} .
$$

Therefore, we obtain

$$
\sup _{t \in[0, T]}\|u(t)\|_{H^{s}} \leqslant M^{-s} \sup _{t \in[0, T]}\|u(t)\|_{H_{M}^{s}} \lesssim \varepsilon_{0}^{s /\left(s-s_{0}\right)}\left(2^{T} B\right)^{1-s /\left(s-s_{0}\right)}
$$

for any $T>0$. This proves (6.11).

REMARK 6.7. In Proposition 6.6, we only obtain an exponential upper bound for the growth of the $H^{s}$-norm. One may upgrade this exponential bound to a polynomial bound if one incorporates a scaling in the argument (as in [27]). We, however, do not pursue this issue since (i) our argument with one parameter $M \geqslant 1$ (without a scaling parameter) suffices to prove global existence and (ii) a polynomial bound is by no mean optimal.

\section{Uniqueness and continuous dependence}

In Section 6, we proved local and global existence of solutions to the Wick ordered cubic 4NLS (1.5). The remaining part of this paper is devoted to the proof of Theorem 1.5. The main difficulty lies in proving uniqueness of solutions. Once we prove uniqueness, continuous dependence follows immediately. See Section 7.2.

In Section 7.1, we set up an energy estimate for the difference of two solutions with the same initial condition. In particular, we state a key identity, expanding the energy estimate into a sum of infinite series of multilinear expressions of arbitrarily large degrees (Propositions 7.1 and 7.2). See Remark 8.20. This identity allows us to establish crucial smoothing estimates. In Section 7.2, we use this proposition to prove Theorem 1.5, in particular uniqueness. The proofs of Propositions 7.1 and 7.2 are somewhat lengthy, involving an infinite iteration of normal form reductions. We therefore postpone the proof of Propositions 7.1 and 7.2 to Section 8 .

7.1. Energy estimate on the difference of two solutions. In this subsection, we consider an energy estimate for the difference of two solutions. As pointed 
out in Remark 5.1, there are two main sources of difficulty: (i) the resonant contribution for the difference of solutions does not vanish and (ii) the symmetrization process in (5.2) and (5.3) (for handling the nonresonant contribution) fails for the difference of solutions; see (7.1).

Let us consider an energy estimate for the difference of two solutions with the same initial condition. Given $u_{0} \in H^{s}(\mathbb{T}), s>-\frac{9}{20}$, let $u$ and $v$ be two solutions to (1.5) constructed in Section 6 with the same initial condition $\left.u\right|_{t=0}=\left.v\right|_{t=0}=u_{0}$. Then, we have $u, v \in C\left([-T, T] ; H^{s}(\mathbb{T})\right) \cap F^{s, \alpha}(T)$ for some $T=T\left(\left\|u_{0}\right\|_{H^{s}}\right)>0$. See Remark 6.4. Using the equation (1.5), we have

$$
\begin{aligned}
& \frac{d}{d t}\|u(t)-v(t)\|_{H^{s}}^{2}=\frac{d}{d t} \sum_{n \in \mathbb{Z}}\langle n\rangle^{2 s}\left|\widehat{u}_{n}-\widehat{v}_{n}\right|^{2} \\
& =2 \operatorname{Re} \sum_{n \in \mathbb{Z}}\langle n\rangle^{2 s} \frac{d}{d t}\left(\widehat{u}_{n}-\widehat{v}_{n}\right) \cdot \overline{\left(\widehat{u}_{n}-\widehat{v}_{n}\right)} \\
& =-2 \operatorname{Re} i \sum_{n \in \mathbb{Z}}\langle n\rangle^{2 s}\left[\widehat{\mathcal{N}(u)_{n}}-\widehat{\mathcal{N}(v)_{n}}\right] \overline{\left(\widehat{u}_{n}-\widehat{v}_{n}\right)} \\
& +2 \operatorname{Re} i \sum_{n \in \mathbb{Z}}\langle n\rangle^{2 s}\left[{\widehat{\mathcal{R}(u)_{n}}}_{n}-\widehat{\mathcal{R}(v)_{n}}\right] \overline{\left(\widehat{u}_{n}-\widehat{v}_{n}\right)} \\
& =-2 \operatorname{Re} i \sum_{n \in \mathbb{Z}}\langle n\rangle^{2 s}\left[\widehat{\mathcal{N}(u)_{n}}-\widehat{\mathcal{N}(v)_{n}}\right] \overline{\left(\widehat{u}_{n}-\widehat{v}_{n}\right)} \\
& -2 \operatorname{Re} i \sum_{n \in \mathbb{Z}}\langle n\rangle^{2 s}\left(\left|\widehat{u}_{n}\right|^{2}-\left|\widehat{v}_{n}\right|^{2}\right) \overline{\left(\widehat{u}_{n}-\widehat{v}_{n}\right)} \widehat{v}_{n} \\
& =: \mathrm{I}+\mathrm{II} \text {, }
\end{aligned}
$$

where $\mathcal{N}(u)$ and $\mathcal{R}(u)$ are as in (1.7) and (1.8).

We first discuss how to handle the main difficulty (i). The main idea is to perform normal form reductions infinitely many times and express

$$
\left|\widehat{u}_{n}(t)\right|^{2}-\left|\widehat{v}_{n}(t)\right|^{2}=\left(\left|\widehat{u}_{n}(t)\right|^{2}-\left|\widehat{u}_{n}(0)\right|^{2}\right)-\left(\left|\widehat{v}_{n}(t)\right|^{2}-\left|\widehat{u}_{n}(0)\right|^{2}\right)
$$

in (7.1) as the difference of sums of multilinear forms of arbitrarily large degrees.

Proposition 7.1. Let $s>-\frac{1}{3}$. Then, there exist multilinear forms $\left\{\mathcal{N}_{0}^{(j)}\right\}_{j=2}^{\infty}$, $\left\{\mathcal{R}^{(2)}\right\}_{j=2}^{\infty}$, and $\left\{\mathcal{N}_{1}^{(j)}\right\}_{j=1}^{\infty}$, depending on a parameter $K>0$, such that

$$
\begin{aligned}
\left|\widehat{u}_{n}(t)\right|^{2}-\left|\widehat{u}_{n}(0)\right|^{2}= & \left.\sum_{j=2}^{\infty} \mathcal{N}_{0}^{(j)}(u)\left(n, t^{\prime}\right)\right|_{0} ^{t} \\
& +\int_{0}^{t}\left[\sum_{j=2}^{\infty} \mathcal{R}^{(j)}(u)\left(n, t^{\prime}\right)+\sum_{j=1}^{\infty} \mathcal{N}_{1}^{(j)}(u)\left(n, t^{\prime}\right)\right] d t
\end{aligned}
$$


for any solution $u \in C\left(\mathbb{R} ; H^{s}(\mathbb{T})\right)$ to (1.5) with smooth (local-in-time) approximations. (Namely, given $t_{0} \in \mathbb{R}$, there exists a sequence of smooth solutions $\left\{u_{N}\right\}_{N \in \mathbb{N}}$ to (1.5) and an interval $I \ni t_{0}$ such that $u_{N}$ tends to $u$ in $C\left(I ; H^{s}(\mathbb{T})\right)$ as $N \rightarrow \infty$.) Here, $\mathcal{N}_{0}^{(j)}$ is a $2 j$-linear form, while $\mathcal{R}^{(j)}$ and $\mathcal{N}_{1}^{(j)}$ are $(2 j+2)$-linear forms (depending on $t \in \mathbb{R}$ ), satisfying the following bounds on $H^{s}(\mathbb{T})$; given any $\theta \in\left(0, \frac{2}{3}\right]$, there exist functions $C_{0, j}, C_{r, j}, C_{1, j}: \mathbb{R}_{+} \rightarrow \mathbb{R}_{+}$, depending on $s$ and $\theta$, such that

$$
\begin{aligned}
& \sum_{n \in \mathbb{Z}}\left\|\mathcal{N}_{0}^{(j)}\left(f_{1}, f_{2}, \ldots, f_{2 j}\right)(n)\right\|_{L_{t}^{\infty}(\mathbb{R})} \lesssim C_{0, j} \prod_{i=1}^{2 j}\left\|f_{i}\right\|_{H^{s}}, \\
& \sum_{n \in \mathbb{Z}}\left\|\mathcal{R}^{(j)}\left(f_{1}, f_{2}, \ldots, f_{2 j}\right)(n)\right\|_{L_{t}^{\infty}(\mathbb{R})} \lesssim C_{r, j} \prod_{i=1}^{2 j+2}\left\|f_{i}\right\|_{H^{s}}, \\
& \sum_{n \in \mathbb{Z}}\left\|\mathcal{N}_{1}^{(j)}\left(f_{1}, f_{2}, \ldots, f_{2 j}\right)(n)\right\|_{L_{t}^{\infty}(\mathbb{R})} \lesssim C_{1, j} \prod_{i=1}^{2 j+2}\left\|f_{i}\right\|_{H^{s}},
\end{aligned}
$$

for any $f_{i} \in H^{s}(\mathbb{T})$ and $K>0$, where

$$
\begin{aligned}
& C_{0, j}(K)= \begin{cases}K^{\max (-1 / 2,-1-2 s)} & \text { if } j=2, \\
K^{-((j-1)(1-\theta)) / 2} o\left(j^{-2}\right) & \text { if } j \geqslant 3,\end{cases} \\
& C_{r, j}(K)= \begin{cases}K^{\max (-1 / 2,-1-3 s)} & \text { if } j=2, \\
K^{-((j-3)(1-\theta)) / 2} o\left(j^{-2}\right) & \text { if } j \geqslant 3,\end{cases} \\
& C_{1, j}(K)= \begin{cases}K^{1 / 2-2 s} & \text { if } j=1, \\
K^{-((j-2)(1-\theta)) / 2} o\left(j^{-2}\right) & \text { if } j \geqslant 2 .\end{cases}
\end{aligned}
$$

It follows from the proof presented in Section 8 that the decay in $j$ is much faster than $j^{-2}$ but it suffices for our purpose in taking double difference in (8.60).

Proposition 7.1 exhibits a smoothing property analogous to Takaoka and Tsutsumi [39] in the context of the modified $\mathrm{KdV}$ on $\mathbb{T}$. In [39], Takaoka and Tsutsumi performed a normal form reduction (= integration by parts) once. See also Nakanishi et al. [32] and Molinet et al. [31], where the authors applied normal form reductions twice in obtaining effective energy estimates for the modified $\mathrm{KdV}$ on $\mathbb{T}$. In order to maximize the smoothing effect, however, we instead perform normal form reductions infinitely many times and re-express $\left|\widehat{u}_{n}(t)\right|^{2}-\left|\widehat{u}_{n}(0)\right|^{2}$ as a sum of infinite series of multilinear forms of arbitrarily large degrees.

Next, we turn our attention to the nonresonant part I in (7.1). In this case, we cannot apply the symmetrization argument as in Section 5. A straightforward 
energy estimate in terms of the $F^{s, \alpha}(T)$-norm without symmetrization works only for $s>-\frac{3}{10}$. See Remarks 7.3 and 8.21. In the following, we apply an infinite iteration of normal form reductions to estimate the nonresonant part I in (7.1) and express I as a sum of infinite series consisting of multilinear terms in $u$ and $v$. The following proposition follows as a corollary to Proposition 7.1. See Section 8.6 for the proof.

Proposition 7.2. Let $s>-\frac{1}{3}$. Then, there exists $T=T\left(\left\|u_{0}\right\|_{H^{s}}\right)>0$ such that

$$
\left|\int_{0}^{t} \mathrm{I}\left(t^{\prime}\right) d t^{\prime}\right| \leqslant \frac{1}{4}\|u-v\|_{C_{T} H^{s}}^{2}
$$

for any $t \in[-T, T]$ and any two solutions $u, v \in C\left([-T, T] ; H^{s}(\mathbb{T})\right) \cap F^{s, \alpha}(T)$ to $(1.5)$ constructed in Section 6 with $\left.u\right|_{t=0}=\left.v\right|_{t=0}=u_{0} \in H^{s}(\mathbb{T})$. (As in Proposition 7.1, it suffices to assume that $u, v \in C\left([-T, T] ; H^{s}(\mathbb{T})\right)$ are two solutions with smooth (local-in-time) approximations.)

We postpone the proof of Propositions 7.1 and 7.2 to Section 8. In the next subsection, we present the proof of Theorem 1.5, assuming Propositions 7.1 and 7.2.

7.2. Uniqueness and continuous dependence. In this subsection, we use Propositions 7.1 and 7.2 to prove Theorem 1.5. Given $s>-\frac{1}{3}$, let $u, v \in C([-T$, $\left.T] ; H^{s}(\mathbb{T})\right) \cap F^{s, \alpha}(T)$ be two solutions to (1.5) constructed in Section 6 with the same initial condition $\left.u\right|_{t=0}=\left.v\right|_{t=0}=u_{0} \in H^{s}(\mathbb{T})$, satisfying

$$
\|u\|_{C_{T} H^{s}},\|v\|_{C_{T} H^{s}} \leqslant r
$$

for some $r>0$. Then, it follows from Proposition 7.1 and the multilinearity of $\mathcal{N}_{0}^{(j)}, \mathcal{R}^{(j)}$, and $\mathcal{N}_{1}^{(j)}$ that

$$
\begin{aligned}
\sup _{n \in \mathbb{Z}} & \left\|\left|\widehat{u}_{n}\right|^{2}-\left|\widehat{v}_{n}\right|^{2}\right\|_{L_{T}^{\infty}} \leqslant \sum_{n \in \mathbb{Z}}\left\|\left|\widehat{u}_{n}\right|^{2}-\left|\widehat{v}_{n}\right|^{2}\right\|_{L_{T}^{\infty}} \\
= & \sum_{n \in \mathbb{Z}}\left\|\left(\left|\widehat{u}_{n}\right|^{2}-\left|\widehat{u}_{n}(0)\right|^{2}\right)-\left(\left|\widehat{v}_{n}\right|^{2}-\left|\widehat{u}_{n}(0)\right|^{2}\right)\right\|_{L_{T}^{\infty}} \\
\leqslant & \sum_{n \in \mathbb{Z}} \sum_{j=2}^{\infty}\left\|\mathcal{N}_{0}^{(j)}(u)(n)-\mathcal{N}_{0}^{(j)}(v)(n)\right\|_{L_{T}^{\infty}} \\
& +T \sum_{n \in \mathbb{Z}} \sum_{j=2}^{\infty}\left\|\mathcal{R}^{(j)}(u)(n)-\mathcal{R}^{(j)}(v)(n)\right\|_{L_{T}^{\infty}}
\end{aligned}
$$




$$
\begin{aligned}
& +T \sum_{n \in \mathbb{Z}} \sum_{j=1}^{\infty}\left\|\mathcal{N}_{1}^{(j)}(u)(n)-\mathcal{N}_{1}^{(j)}(v)(n)\right\|_{L_{T}^{\infty}} \\
& \lesssim K^{\max (-1 / 2,-1-2 s)} r^{3}\|u-v\|_{C_{T} H^{s}}+\sum_{j=3}^{\infty} K^{-((j-1)(1-\theta)) / 2} r^{2 j-1}\|u-v\|_{C_{T} H^{s}} \\
& +T K^{\max (-1 / 2,-1-3 s)} r^{5}\|u-v\|_{C_{T} H^{s}}+T \sum_{j=3}^{\infty} K^{-((j-3)(1-\theta)) / 2} r^{2 j+1}\|u-v\|_{C_{T} H^{s}} \\
& +T K^{1 / 2-2 s} r^{3}\|u-v\|_{C_{T} H^{s}}+T \sum_{j=2}^{\infty} K^{-((j-2)(1-\theta)) / 2} r^{2 j+1}\|u-v\|_{C_{T} H^{s}}
\end{aligned}
$$

Then, by first choosing $K=K(r)>0$ sufficiently large and then choosing $T=$ $T(K)=T(r)>0$ sufficiently small, we conclude that

$$
\sup _{n \in \mathbb{Z}}\left\|\left|\widehat{u}_{n}\right|^{2}-\left|\widehat{v}_{n}\right|^{2}\right\|_{L_{T}^{\infty}} \leqslant \sum_{n \in \mathbb{Z}}\left\|\left|\widehat{u}_{n}\right|^{2}-\left|\widehat{v}_{n}\right|^{2}\right\|_{L_{T}^{\infty}} \leqslant \frac{1}{16 r}\|u-v\|_{C_{T} H^{s}} .
$$

Hence, it follows from (7.6) and Cauchy-Schwarz inequality that

$$
\begin{aligned}
\left|\int_{0}^{T} \mathrm{II}(t) d t\right| & \leqslant T\|\mathrm{II}\|_{L_{T}^{\infty}}=2 T\left\|\sum_{n \in \mathbb{Z}}\langle n\rangle^{2 s}\left(\left|\widehat{u}_{n}\right|^{2}-\left|\widehat{v}_{n}\right|^{2}\right) \overline{\left(\widehat{u}_{n}-\widehat{v}_{n}\right)} \widehat{v}_{n}\right\|_{L_{T}^{\infty}} \\
& \leqslant \frac{1}{4}\|u-v\|_{C_{T} H^{s}}^{2} .
\end{aligned}
$$

Therefore, by integrating (7.1) from 0 to $T$ with $u(0)=v(0)$ and applying Proposition 7.2 and (7.7), we obtain

$$
\|u-v\|_{C_{T} H^{s}}^{2} \leqslant \frac{1}{2}\|u-v\|_{C_{T} H^{s}}^{2} .
$$

This proves local-in-time uniqueness of solutions to (1.5) in $C\left([-T, T] ; H^{s}(\mathbb{T})\right) \cap$ $F^{s, \alpha}(T)$ with some $T=T\left(\left\|u_{0}\right\|_{H^{s}}\right)>0$. In view of the global-in-time bound in Proposition 6.6, we can iterate this argument and establish uniqueness globally in time. Here, uniqueness holds in

$$
\bigcap_{t \in \mathbb{R}}\left\{u \in C\left(\mathbb{R} ; H^{s}(\mathbb{T})\right) ; u(\cdot-t) \in F^{s, \alpha}\left(T\left(t, u_{0}\right)\right)\right\}
$$

for some appropriate $T\left(t, u_{0}\right)>0$. (Since we only need Propositions 7.1 and 7.2, the uniqueness holds among the solutions in $C\left(\mathbb{R} ; H^{s}(\mathbb{T})\right)$ with smooth approximations. Note that in such a class, uniqueness is by no means automatic since we do not have continuous dependence (at this point).) 
REMARK 7.3. (i) We stress that it is crucial that $u$ and $v$ have the same initial condition in the argument above.

(ii) We can estimate the nonresonant contribution $I$ in (7.1) in terms of the $F^{s, \alpha}(T)$-norm for $s>-\frac{3}{10}$. See Remark 8.21 below. This provides uniqueness for a more restrictive range $s>-\frac{3}{10}$.

Note that an energy estimate of the form:

$\|u-v\|_{E^{s}(T)}^{2} \lesssim\|u(0)-v(0)\|_{H^{s}}^{2}+T^{\theta} C\left(\|u\|_{F^{s, \alpha}(T)},\|v\|_{F^{s, \alpha}(T)}\right)\|u-v\|_{F^{s, \alpha}(T)}^{2}$

for two solutions $u$ and $v$ with different initial data $u(0) \neq v(0)$ is false for $s<0$ in view of the failure of local uniform continuity for the solution map for (1.5) in negative Sobolev spaces.

(iii) By combining the proofs of Propositions 7.1 and 7.2, we can express $\|u(t)-v(t)\|_{H^{s}}^{2}$ as a sum of infinite series consisting of multilinear linear terms (in $u$ and $v$ ) of arbitrarily large degrees. Moreover, thanks to the multilinearity of the summands and the double difference structure of I and II, we can rearrange the series so that we can extract two factors of (the Fourier coefficient of) $u-v$ in each of the multilinear terms. See Remark 8.20.

Thanks to the uniqueness of solutions, continuous dependence of the solution map for (1.5) on initial data in $H^{s}(\mathbb{T})$ basically follows from repeating the argument in Section 6.

LEMMA 7.4. Given $s>-\frac{1}{3}$, let $\left\{u_{n}\right\}_{n \in \mathbb{N}}$ and $u$ are the unique solutions to (1.5) in $C\left(\mathbb{R} ; H^{s}(\mathbb{T})\right)$ with $\left.u_{n}\right|_{t=0}=u_{0, n}$ and $\left.u\right|_{t=0}=u_{0}$. If we have

$$
\lim _{n \rightarrow \infty}\left\|u_{0, n}-u_{0}\right\|_{H^{s}}=0
$$

then we have

$$
\lim _{n \rightarrow \infty}\left\|u_{n}-u\right\|_{C_{T} H^{s}}=0
$$

for any $T>0$.

Proof. It suffices to prove (7.8) for sufficiently small $T>0$ since the general case follows from iterating local-in-time arguments in view of the global-in-time bound in Proposition 6.6. Let $T=T\left(\left\|u_{0}\right\|_{H^{s}}\right)>0$ be the local existence time from Section 6 for initial data of size $\left\|u_{0}\right\|_{H^{s}}+1$. Without loss of generality, we assume that $\sup _{n \in \mathbb{Z}}\left\|u_{0, n}\right\|_{H^{s}} \leqslant\left\|u_{0}\right\|_{H^{s}}+1$. 
Note that it suffices to prove that $\left\{u_{n}\right\}_{n \in \mathbb{N}}$ is precompact in $C([-T, T]$; $\left.H^{s}(\mathbb{T})\right) \cap F^{s, \alpha}(T)$. This implies that any subsequence of $\left\{u_{n}\right\}_{n \in \mathbb{N}}$ has a convergent subsubsequence. In view of convergence to $u_{0}$ at time 0 and the uniqueness of solutions, such a convergent subsubsequence must converge to $u$ since it converges to $u_{0}$ at time 0 . Therefore, the entire sequence $\left\{u_{n}\right\}_{n \in \mathbb{N}}$ converges to $u$ in $C\left([-T, T] ; H^{s}(\mathbb{T})\right)$, yielding $(7.8)$.

Since $u_{0, n}$ converges to $u_{0}$ in $H^{s}(\mathbb{T})$, we see that $\left\{u_{0, n}\right\}_{n \in \mathbb{N}} \cup\left\{u_{0}\right\}$ is compact in $H^{s}(\mathbb{T})$. Then, by Riesz' characterization of compactness, given $\varepsilon>0$, there exists $N \in \mathbb{N}$ such that

$$
\left\|\mathbf{P}_{>N} u_{0, n}\right\|_{H^{s}}<\varepsilon \quad \text { and } \quad\left\|\mathbf{P}_{>N} u_{0}\right\|_{H^{s}}<\varepsilon
$$

for all $n \in \mathbb{N}$. Then, by exploiting the smoothing property of the energy estimate (5.4) in Proposition 5.2 (see Remark 5.3) as in [19, Lemma 8.2], we claim that, given $\varepsilon>0$, there exists $N_{0} \in \mathbb{N}$ such that

$$
\left\|\mathbf{P}_{>N} u_{n}\right\|_{C_{T} H^{s}}<\varepsilon
$$

for all $N \geqslant N_{0}$, uniformly in $n \in \mathbb{N}$. In view of Remark 5.3, it follows from (the proof of) Proposition 5.2 with the a priori bound:

$$
\|u\|_{L^{\infty}\left([-T, T] ; H^{s}\right)} \lesssim\|u\|_{F^{s, \alpha}(T)} \leqslant 2\left\|u_{0}\right\|_{H^{s}}
$$

that there exists small $\delta>0$ such that

$$
\begin{aligned}
\|\| \mathbf{P}_{>N} u_{n}\left\|_{E^{s}(T)}^{2}-\right\| \mathbf{P}_{>N} u_{0, n} \|_{H^{s}}^{2} \mid & \lesssim T^{\theta}\left\|\mathbf{P}_{>c N} u_{n}\right\|_{F^{s-\delta, \alpha}(T)}^{2}\left\|u_{n}\right\|_{F^{s-\delta, \alpha}(T)}^{2} \\
& \lesssim C\left(\left\|u_{0}\right\|_{H^{s}}\right) N^{-2 \delta} \longrightarrow 0,
\end{aligned}
$$

as $N \rightarrow \infty$, uniformly in $n \in \mathbb{N}$. Note that the a priori bound (7.11) follows from Lemma 2.2 and (6.6). Hence, from (7.9), there exists $N_{0} \in \mathbb{N}$ such that

$$
\left\|\mathbf{P}_{>N} u_{n}\right\|_{C_{T} H^{s}}^{2} \leqslant\left\|\mathbf{P}_{>N} u_{n}\right\|_{E^{s}(T)}^{2} \lesssim\left\|\mathbf{P}_{>N} u_{0, n}\right\|_{H^{s}}^{2}+C\left(\left\|u_{0}\right\|_{H^{s}}\right) N^{-2 \delta} \lesssim \varepsilon
$$

for all $N \geqslant N_{0}$, uniformly in $n \in \mathbb{N}$. This proves (7.10).

Fix $\varepsilon>0$. By (7.10), there exists $N_{0}>0$ such that $\left\|\mathbf{P}_{>N_{0}} u_{n}\right\|_{C_{T} H^{s}}<\frac{\varepsilon}{3}$ for all $n \in$ $\mathbb{N}$. Arguing as in the proof of Lemma 8.2 in [19] with Ascoli-Arzelà compactness theorem, we conclude that $\left\{\mathbf{P}_{\leqslant N_{0}} u_{n}\right\}_{n \in \mathbb{N}}$ is precompact in $C([-T, T]$; $H^{s}(\mathbb{T})$ ). Hence, there exists a finite cover by balls of radius $\varepsilon / 3$ (in $C_{T} H^{s}$ ) centered at $\left\{\mathbf{P}_{\leqslant N_{0}} u_{n_{k}}\right\}_{k=1}^{K}$. Then, the balls of radius $\varepsilon$ (in $C_{T} H^{s}$ ) centered at $\left\{u_{n_{k}}\right\}_{k=1}^{K}$ cover $\left\{u_{n}\right\}_{n \in \mathbb{N}}$. This proves the precompactness of $\left\{u_{n}\right\}_{n \in \mathbb{N}}$ in $C([-T, T]$; $\left.H^{s}(\mathbb{T})\right)$.

Let us extract a subsequence, still denoted by $\left\{u_{n}\right\}_{n \in \mathbb{N}}$, converging to some $u$ in $C\left([-T, T] ; H^{s}(\mathbb{T})\right)$. In view of the uniform tail estimate (7.12), this subsequence 
also converges in $E^{s}(T)$. Then, by making $T$ smaller, if necessary, it follows from (6.3) and (6.4) that

$$
\left\|u_{n}-u_{m}\right\|_{F^{s, \alpha}(T)} \lesssim\left\|u_{n}-u_{m}\right\|_{E^{s}(T)} .
$$

Hence, $\left\{u_{n}\right\}$ converges to $u$ in $F^{s, \alpha}(T)$.

\section{Normal form reductions}

It remains to prove Propositions 7.1 and 7.2. In this section, we perform an infinite iteration of normal form reductions and present the proofs of these propositions in Sections 8.5 and 8.6.

Let $u$ be a smooth global solution to the Wick ordered cubic 4NLS (1.5) and $\mathbf{u}(t)=S(-t) u(t)$ be its interaction representation defined in (2.1). Then, by the fundamental theorem of calculus with (2.2), we can write the growth of the energy quantity $\left|\widehat{u}_{n}(t)\right|^{2}$ as

$$
\begin{aligned}
\left|\widehat{u}_{n}(t)\right|^{2}-\left|\widehat{u}_{n}(0)\right|^{2} & =\left|\widehat{\mathbf{u}}_{n}(t)\right|^{2}-\left|\widehat{\mathbf{u}}_{n}(0)\right|^{2} \\
& =-2 \operatorname{Re} i\left(\int_{0}^{t} \sum_{\Gamma(n)} e^{-i \phi(\bar{n}) t^{\prime}} \widehat{\mathbf{u}}_{n_{1}} \widehat{\mathbf{u}}_{n_{2}} \widehat{\mathbf{u}}_{n_{3}} \widehat{\mathbf{u}}_{n}\left(t^{\prime}\right) d t^{\prime}\right) .
\end{aligned}
$$

(The quantity $\left|\widehat{\mathbf{u}}_{n}(t)\right|^{2}$ is often referred to as an action.) Integrating by parts in time,

$$
\begin{aligned}
= & \left.2 \operatorname{Re}\left(\sum_{\Gamma(n)} \frac{e^{-i \phi(\bar{n}) t}}{\phi(\bar{n})} \widehat{\mathbf{u}}_{n_{1}} \widehat{\mathbf{u}}_{n_{2}} \widehat{\mathbf{u}}_{n_{3}} \widehat{\mathbf{u}}_{n}\right)\right|_{0} ^{t} \\
& -2 \operatorname{Re}\left(\int_{0}^{t} \sum_{\Gamma(n)} \frac{e^{-i \phi(\bar{n}) t}}{\phi(\bar{n})} \partial_{t}\left(\widehat{\mathbf{u}}_{n_{1}} \widehat{\mathbf{u}}_{n_{2}} \widehat{\mathbf{u}}_{n_{3}} \widehat{\mathbf{u}}_{n}\right)\left(t^{\prime}\right) d t^{\prime}\right) .
\end{aligned}
$$

In view of the factorization (2.4), we see that the gain of $\phi(\bar{n})$ in the denominators corresponds to the gain of derivatives. The price to pay here is that the second term on the right-hand side of (8.1) is now 6-linear. In order to handle the last term in (8.1), we need to apply an integration by parts again, yielding 8-linear terms. In fact, we iterate this procedure infinitely many times in the following. When we apply integration by parts in an iterative manner, the time derivative may fall on any of the factors, generating higher order nonlinear terms. (In the following, we perform integration by parts without integration symbols, which we refer to as differentiation by parts, following [1]. Moreover, we perform integration by parts only in the case the phase factor is 'sufficiently large'.) We need to keep track of all possible ways in which the time derivatives fall and sum over the 
contributions from all possible choices. This can be a combinatorially challenging task. In order to handle multilinear terms of increasing complexity appearing in the infinite iteration of normal form reductions, we introduce the notion of ordered bi-trees in the following.

8.1. Ordered bi-trees. In [18], the first author implemented an infinite iteration of normal form reductions to study the cubic NLS on $\mathbb{T}$, where differentiation by parts was applied to the evolution equation satisfied by the interaction representation. In [18], (ternary) trees and ordered trees played an important role for indexing such terms and frequencies arising in the general steps of normal form reductions.

In the following, we instead implement an infinite iteration scheme of normal form reductions applied to the energy quantity $\left|\widehat{\mathbf{u}}_{n}(t)\right|^{2}$. More precisely, to the evolution equation satisfied by the energy quantity. In particular, we need treelike structures that grow in two directions. For this purpose, we introduce the notion of bi-trees and ordered bi-trees in the following. Once we replace trees and ordered trees by bi-trees and ordered bi-trees, other related notions can be defined in a similar manner as in [18] with certain differences to be noted.

DEFINITION 8.1. (i) Given a partially ordered set $\mathcal{T}$ with partial ordering $\leqslant$, we say that $b \in \mathcal{T}$ with $b \leqslant a$ and $b \neq a$ is a child of $a \in \mathcal{T}$, if $b \leqslant c \leqslant a$ implies either $c=a$ or $c=b$. If the latter condition holds, we also say that $a$ is the parent of $b$.

(ii) A tree $\mathcal{T}$ is a finite partially ordered set satisfying the following properties:

(a) Let $a_{1}, a_{2}, a_{3}, a_{4} \in \mathcal{T}$. If $a_{4} \leqslant a_{2} \leqslant a_{1}$ and $a_{4} \leqslant a_{3} \leqslant a_{1}$, then we have $a_{2} \leqslant a_{3}$ or $a_{3} \leqslant a_{2}$.

(b) A node $a \in \mathcal{T}$ is called terminal, if it has no child. A nonterminal node $a \in \mathcal{T}$ is a node with exactly three ordered children denoted by $a_{1}, a_{2}$, and $a_{3}$.

(c) There exists a maximal element $r \in \mathcal{T}$ (called the root node) such that $a \leqslant r$ for all $a \in \mathcal{T}$.

(d) $\mathcal{T}$ consists of the disjoint union of $\mathcal{T}^{0}$ and $\mathcal{T}^{\infty}$, where $\mathcal{T}^{0}$ and $\mathcal{T}^{\infty}$ denote the collections of nonterminal nodes and terminal nodes, respectively.

(iii) A bi-tree $\mathcal{T}=\mathcal{T}_{1} \cup \mathcal{T}_{2}$ is a union of two trees $\mathcal{T}_{1}$ and $\mathcal{T}_{2}$, where the root nodes $r_{j}$ of $\mathcal{T}_{j}, j=1,2$, are joined by an edge. A bi-tree $\mathcal{T}$ consists of the disjoint union of $\mathcal{T}^{0}$ and $\mathcal{T}^{\infty}$, where $\mathcal{T}^{0}$ and $\mathcal{T}^{\infty}$ denote the collections 

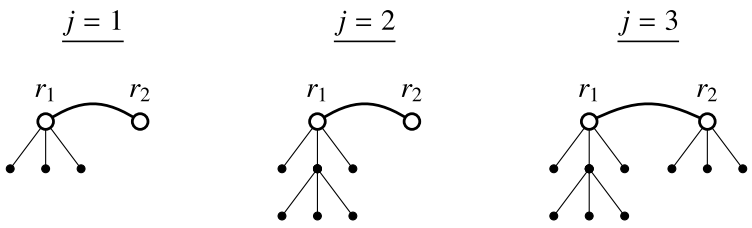

Figure 1. Examples of bi-trees of the $j$ th generations, $j=1,2,3$.

of nonterminal nodes and terminal nodes, respectively. By convention, we assume that the root node $r_{1}$ of the first tree $\mathcal{T}_{1}$ is nonterminal, while the root node $r_{2}$ of the second tree $\mathcal{T}_{2}$ may be terminal.

(iv) Given a bi-tree $\mathcal{T}=\mathcal{T}_{1} \cup \mathcal{T}_{2}$, we define a projection $\Pi_{j}, j=1$, 2, onto a tree by setting

$$
\Pi_{j}(\mathcal{T})=\mathcal{T}_{j}
$$

In Figure $1, \Pi_{1}(\mathcal{T})$ corresponds to the tree on the left under the root node $r_{1}$, while $\Pi_{2}(\mathcal{T})$ corresponds to the tree on the right under the root node $r_{2}$.

Note that the number $|\mathcal{T}|$ of nodes in a bi-tree $\mathcal{T}$ is $3 j+2$ for some $j \in \mathbb{N}$, where $\left|\mathcal{T}^{0}\right|=j$ and $\left|\mathcal{T}^{\infty}\right|=2 j+2$. Let us denote the collection of trees of the $j$ th generation (namely, with $j$ parental nodes) by $B T(j)$, that is,

$$
B T(j):=\{\mathcal{T}: \mathcal{T} \text { is a bi-tree with }|\mathcal{T}|=3 j+2\}
$$

Next, we introduce the notion of ordered bi-trees, for which we keep track of how a bi-tree 'grew' into a given shape.

DEFINITION 8.2. We say that a sequence $\left\{\mathcal{T}_{j}\right\}_{j=1}^{J}$ is a chronicle of $J$ generations, if

(a) $\mathcal{T}_{j} \in B T(j)$ for each $j=1, \ldots, J$;

(b) $\mathcal{T}_{j+1}$ is obtained by changing one of the terminal nodes in $\mathcal{T}_{j}$ into a nonterminal node (with three children), $j=1, \ldots, J-1$.

Given a chronicle $\left\{\mathcal{T}_{j}\right\}_{j=1}^{J}$ of $J$ generations, we refer to $\mathcal{T}_{J}$ as an ordered bi-tree of the $J$ th generation. We denote the collection of the ordered trees of the $J$ th generation by $\mathfrak{B T}(J)$. Note that the cardinality of $\mathfrak{B T}(J)$ is given by $|\mathfrak{B T}(1)|=1$ and

$$
|\mathfrak{B T}(J)|=4 \cdot 6 \cdot 8 \cdots 2 J=2^{J-1} \cdot J !=: c_{J}, \quad J \geqslant 2 .
$$


We stress that the notion of ordered bi-trees comes with associated chronicles. For example, given two ordered bi-trees $\mathcal{T}_{J}$ and $\widetilde{\mathcal{T}}_{J}$ of the $J$ th generation, it may happen that $\mathcal{T}_{J}=\widetilde{\mathcal{T}}_{J}$ as bi-trees (namely as planar graphs) according to Definition 8.1, while $\mathcal{T}_{J} \neq \widetilde{\mathcal{T}}_{J}$ as ordered bi-trees according to Definition 8.2. In the following, when we refer to an ordered bi-tree $\mathcal{T}_{J}$ of the $J$ th generation, it is understood that there is an underlying chronicle $\left\{\mathcal{T}_{j}\right\}_{j=1}^{J}$.

Given a bi-tree $\mathcal{T}$, we associate each terminal node $a \in \mathcal{T}^{\infty}$ with the Fourier coefficient (or its complex conjugate) of the interaction representation $\mathbf{u}$ and sum over all possible frequency assignments. In order to do this, we introduce the index function assigning frequencies to all the nodes in $\mathcal{T}$ in a consistent manner.

DEFINITION 8.3. (i) Given a bi-tree $\mathcal{T}=\mathcal{T}_{1} \cup \mathcal{T}_{2}$, we define an index function $\mathbf{n}: \mathcal{T} \rightarrow \mathbb{Z}$ such that

(a) $n_{r_{1}}=n_{r_{2}}$, where $r_{j}$ is the root node of the tree $\mathcal{T}_{j}, j=1,2$;

(b) $n_{a}=n_{a_{1}}-n_{a_{2}}+n_{a_{3}}$ for $a \in \mathcal{T}^{0}$, where $a_{1}, a_{2}$, and $a_{3}$ denote the children of $a$;

(c) $\left\{n_{a}, n_{a_{2}}\right\} \cap\left\{n_{a_{1}}, n_{a_{3}}\right\}=\emptyset$ for $a \in \mathcal{T}^{0}$,

where we identified $\mathbf{n}: \mathcal{T} \rightarrow \mathbb{Z}$ with $\left\{n_{a}\right\}_{a \in \mathcal{T}} \in \mathbb{Z}^{\mathcal{T}}$. We use $\mathfrak{N}(\mathcal{T}) \subset \mathbb{Z}^{\mathcal{T}}$ to denote the collection of such index functions $\mathbf{n}$ on $\mathcal{T}$.

(ii) Given a tree $\mathcal{T}$, we also define an index function $\mathbf{n}: \mathcal{T} \rightarrow \mathbb{Z}$ by omitting the condition (a) and denote by $\mathfrak{N}(\mathcal{T}) \subset \mathbb{Z}^{\mathcal{T}}$ the collection of index functions $\mathbf{n}$ on $\mathcal{T}$, when there is no confusion.

REMARK 8.4. (i) In view of the consistency condition, we can refer to $n_{r_{1}}=n_{r_{2}}$ as the frequency at the root node without ambiguity. We shall denote it by $n_{r}$.

(ii) Just like index functions for (ordered) trees considered in [18], an index function $\mathbf{n}=\left\{n_{a}\right\}_{a \in \mathcal{T}}$ for a bi-tree $\mathcal{T}$ is completely determined once we specify the values $n_{a} \in \mathbb{Z}$ for the terminal nodes $a \in \mathcal{T}^{\infty}$. An index function $\mathbf{n}$ for a bi-tree $\mathcal{T}=\mathcal{T}_{1} \cup \mathcal{T}_{2}$ is basically a pair $\left(\mathbf{n}_{1}, \mathbf{n}_{2}\right)$ of index functions $\mathbf{n}_{j}$ for the trees $\mathcal{T}_{j}, j=1,2$, (omitting the nonresonance condition in [18, Definition 3.5 (iii)]), satisfying the consistency condition (a): $n_{r_{1}}=n_{r_{2}}$.

(iii) Given a bi-tree $\mathcal{T} \in \mathfrak{B T}(J)$, consider the summation of all possible frequency assignments $\left\{\mathbf{n} \in \mathfrak{N}(\mathcal{T}): n_{r}=n\right\}$. While $\left|\mathcal{T}^{\infty}\right|=2 J+2$, there are $2 J$ free variables in this summation. Namely, the condition $n_{r}=n$ reduces two summation variables. It is easy to see this by separately considering the cases $\Pi_{2}(\mathcal{T})=\left\{r_{2}\right\}$ and $\Pi_{2}(\mathcal{T}) \neq\left\{r_{2}\right\}$. 
Given an ordered bi-tree $\mathcal{T}_{J}$ of the $J$ th generation with a chronicle $\left\{\mathcal{T}_{j}\right\}_{j=1}^{J}$ and associated index functions $\mathbf{n} \in \mathfrak{N}\left(\mathcal{T}_{J}\right)$, we would like to keep track of the 'generations' of frequencies as in [18]. In the following, we use superscripts to denote such generations of frequencies.

Fix $\mathbf{n} \in \mathfrak{N}\left(\mathcal{T}_{J}\right)$. Consider $\mathcal{T}_{1}$ of the first generation. Its nodes consist of the two root nodes $r_{1}, r_{2}$, and the children $r_{11}, r_{12}$, and $r_{13}$ of the first root node $r_{1}$. See Figure 1 . We define the first generation of frequencies by

$$
\left(n^{(1)}, n_{1}^{(1)}, n_{2}^{(1)}, n_{3}^{(1)}\right):=\left(n_{r_{1}}, n_{r_{11}}, n_{r_{12}}, n_{r_{13}}\right) .
$$

From Definition 8.3, we have

$$
n^{(1)}=n_{r_{2}}, \quad n^{(1)}=n_{1}^{(1)}-n_{2}^{(1)}+n_{3}^{(1)}, \quad n_{2}^{(1)} \neq n_{1}^{(1)}, n_{3}^{(1)} .
$$

Next, we construct an ordered bi-tree $\mathcal{T}_{2}$ of the second generation from $\mathcal{T}_{1}$ by changing one of its terminal nodes $a \in \mathcal{T}_{1}^{\infty}=\left\{r_{2}, r_{11}, r_{12}, r_{13}\right\}$ into a nonterminal node. Then, we define the second generation of frequencies by setting

$$
\left(n^{(2)}, n_{1}^{(2)}, n_{2}^{(2)}, n_{3}^{(2)}\right):=\left(n_{a}, n_{a_{1}}, n_{a_{2}}, n_{a_{3}}\right) .
$$

Note that we have $n^{(2)}=n^{(1)}$ or $n_{k}^{(1)}$ for some $k \in\{1,2,3\}$,

$$
n^{(2)}=n_{1}^{(2)}-n_{2}^{(2)}+n_{3}^{(2)}, \quad n_{2}^{(2)} \neq n_{1}^{(2)}, n_{3}^{(2)},
$$

where the last identities follow from Definition 8.3. This extension of $\mathcal{T}_{1} \in \mathfrak{B T}(1)$ to $\mathcal{T}_{2} \in \mathfrak{B T}(2)$ corresponds to introducing a new set of frequencies after the first differentiation by parts, where the time derivative falls on each of $\widehat{\mathbf{u}}_{n}$ and $\widehat{\mathbf{u}}_{n_{j}}, j=$ $1,2,3$. (The complex conjugate signs on $\widehat{\mathbf{u}}_{n}$ and $\widehat{\mathbf{u}}_{n_{j}}$ do not play any significant role. Hereafter, we drop the complex conjugate sign. We also assume that all the Fourier coefficients of $\mathbf{u}$ are nonnegative.)

In general, we construct an ordered bi-tree $\mathcal{T}_{j}$ of the $j$ th generation from $\mathcal{T}_{j-1}$ by changing one of its terminal nodes $a \in \mathcal{T}_{j-1}^{\infty}$ into a nonterminal node. Then, we define the $j$ th generation of frequencies by

$$
\left(n^{(j)}, n_{1}^{(j)}, n_{2}^{(j)}, n_{3}^{(j)}\right):=\left(n_{a}, n_{a_{1}}, n_{a_{2}}, n_{a_{3}}\right) .
$$

As before, it follows from Definition 8.3 that

$$
n^{(j)}=n_{1}^{(j)}-n_{2}^{(j)}+n_{3}^{(j)}, \quad n_{2}^{(j)} \neq n_{1}^{(j)}, n_{3}^{(j)} .
$$

Given an ordered bi-tree $\mathcal{T}$, we denote by $B_{j}=B_{j}(\mathcal{T})$ the set of all possible frequencies in the $j$ th generation. Figure 2 below shows an example of a bi-tree $\mathcal{T} \in \mathfrak{B T}(3)$ ornamented by an index function $\mathbf{n} \in \mathfrak{N}(\mathcal{T})$. 


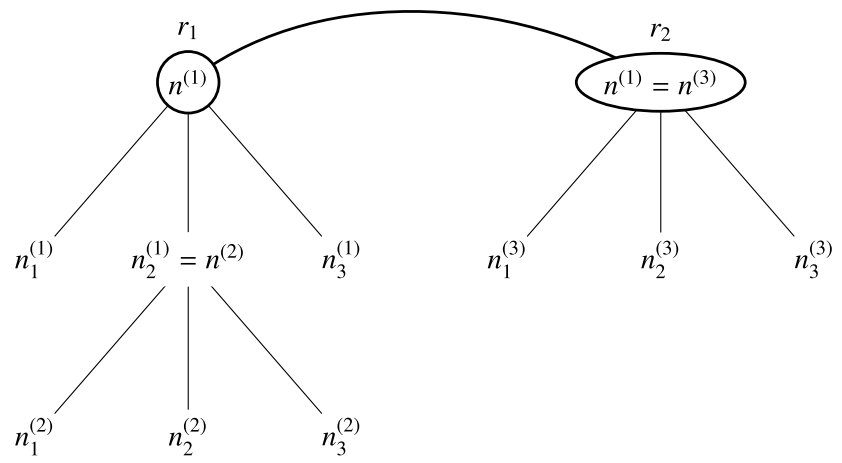

Figure 2. An example of a bi-tree $\mathcal{T} \in \mathfrak{B T}(3)$. Here, we have ornamented the nodes with the values of an index function $\mathbf{n}=\left\{n_{a}\right\}_{a \in \mathcal{T}} \in \mathfrak{N}(\mathcal{T})$, specifying the generations of frequencies as discussed above.

We denote by $\phi_{j}$ the corresponding phase function introduced at the $j$ th generation:

$$
\phi_{j}=\phi_{j}\left(n^{(j)}, n_{1}^{(j)}, n_{2}^{(j)}, n_{3}^{(j)}\right):=\left(n_{1}^{(j)}\right)^{4}-\left(n_{2}^{(j)}\right)^{4}+\left(n_{3}^{(j)}\right)^{4}-\left(n^{(j)}\right)^{4} .
$$

Then, by (2.4), we have

$$
\left|\phi_{j}\right| \sim\left(n_{\max }^{(j)}\right)^{2} \cdot\left|\left(n^{(j)}-n_{1}^{(j)}\right)\left(n^{(j)}-n_{3}^{(j)}\right)\right|,
$$

where $n_{\max }^{(j)}:=\max \left(\left|n^{(j)}\right|,\left|n_{1}^{(j)}\right|,\left|n_{2}^{(j)}\right|,\left|n_{3}^{(j)}\right|\right)$. Finally, we denote by $\mu_{j}$ the phase function (at the $j$ th generation) corresponding to the usual cubic NLS with the second order dispersion:

$$
\begin{aligned}
\mu_{j} & =\mu_{j}\left(n^{(j)}, n_{1}^{(j)}, n_{2}^{(j)}, n_{3}^{(j)}\right):=\left(n_{1}^{(j)}\right)^{2}-\left(n_{2}^{(j)}\right)^{2}+\left(n_{3}^{(j)}\right)^{2}-\left(n^{(j)}\right)^{2} \\
& =-2\left(n^{(j)}-n_{1}^{(j)}\right)\left(n^{(j)}-n_{3}^{(j)}\right) .
\end{aligned}
$$

Note that we have

$$
\left|\phi_{j}\right| \sim\left(n_{\max }^{(j)}\right)^{2} \cdot\left|\mu_{j}\right| \gtrsim\left|\mu_{j}\right|^{2}
$$

8.2. First few steps of normal form reductions. We first implement a formal infinite iteration scheme of normal form reductions for smooth functions without justifying switching of limits and summations. As before, let $u$ be a smooth global solution to (1.5) and $\mathbf{u}(t)=S(-t) u(t)$ be its interaction representation. For simplicity of notations, we simply set $\mathbf{u}_{n}=\widehat{\mathbf{u}}_{n}$ in the following. We may also 
drop the minus signs and the complex number $i$. In the following, we establish various multilinear estimates. Our argument has a common feature with [18] in that Cauchy-Schwarz inequality plays an important role. On the other hand, while the divisor counting argument played a crucial role in [18], we do not use the divisor counting argument in maximizing a gain of derivative. (We, however, use the divisor counting argument to show that the error term converges to 0 , where we do not need to show any gain of derivatives. See Section 8.4.)

Given $s>-\frac{1}{3}$, fix $K=K(s)>0$ (to be chosen later. As we see in Section 8.6, the constant $K$ will also depend on other constants.) Using the notations introduced in the previous subsection, for fixed $n$, we have

$$
\begin{aligned}
& \frac{d}{d t}\left|\mathbf{u}_{n}(t)\right|^{2}=-2 \operatorname{Re} i \sum_{\Gamma(n)} e^{-i \phi(\bar{n}) t} \mathbf{u}_{n_{1}} \overline{\mathbf{u}_{n_{2}}} \mathbf{u}_{n_{3}} \overline{\mathbf{u}_{n}} \\
& =-2 \operatorname{Re} i \sum_{\mathcal{T}_{1} \in \mathfrak{B} \mathfrak{T}(1)} \sum_{\substack{\mathbf{n} \in \mathfrak{N}\left(\mathcal{T}_{1}\right) \\
n_{r}=n}} e^{-i \phi_{1} t} \prod_{a \in \mathcal{T}_{1}^{\infty}} \mathbf{u}_{n_{a}}=: \mathcal{N}^{(1)}(\mathbf{u})_{n},
\end{aligned}
$$

where $\Gamma(n)$ is as in (2.3). (Due to the presence of $e^{-i \phi_{1} t}$, the multilinear form $\mathcal{N}^{(1)}(\mathbf{u})_{n}$ is nonautonomous in $t$. Hence, strictly speaking, we should denote it by $\mathcal{N}^{(1)}(t)(\mathbf{u}(t))_{n}$. In the following, however, we estimate these multilinear forms, uniformly in $t \in \mathbb{R}$, and thus we simply suppress such $t$-dependence when there is no confusion. The same comment applies to other multilinear forms.)

We divide the frequency space into $\left|\phi_{1}\right| \leqslant K$ and $\left|\phi_{1}\right|>K$. Namely, define $A_{K}$ by

$$
A_{K}:=\left\{\mathbf{n} \in \mathfrak{N}\left(\mathcal{T}_{1}\right):\left|\phi_{1}(\mathbf{n})\right| \leqslant K, n_{r}=n\right\}
$$

and write

$$
\mathcal{N}^{(1)}=\mathcal{N}_{1}^{(1)}+\mathcal{N}_{2}^{(1)}
$$

where $\mathcal{N}_{1}^{(1)}$ is the restriction of $\mathcal{N}^{(1)}$ onto $A_{K}$ and $\mathcal{N}_{2}^{(1)}=\mathcal{N}^{(1)}-\mathcal{N}_{1}^{(1)}$. Thanks to the restriction $\left|\phi_{1}\right| \leqslant K$, we can estimate the nearly resonant part $\mathcal{N}_{1}^{(1)}$ as follows.

LEMMA 8.5. Let $\mathcal{N}_{1}^{(1)}$ be as above. Then, for any $s \leqslant 0$, we have

$$
\sum_{n \in \mathbb{Z}}\left|\mathcal{N}_{1}^{(1)}(\mathbf{u})_{n}\right| \lesssim K^{1 / 2-2 s}\|\mathbf{u}\|_{H^{s}}^{4}
$$

REMARK 8.6. In Lemma 8.5, we established an $\ell_{n}^{1}$-bound on $\left\{\mathcal{N}_{1}^{(1)}(\mathbf{u})_{n}\right\}_{n \in \mathbb{Z}}$. In this and the next subsections, we estimate various multilinear terms in the $\ell_{n}^{1}$-norm. We point out that, in proving uniqueness of solutions to (1.5) with the same initial condition, it suffices to estimate these multilinear terms only in the much weaker $\ell_{n}^{\infty}$-norm. Unfortunately, we do not know how to convert this gain in summability to a gain in differentiability to go below $-\frac{1}{3}$. See Lemma 8.10 . 
Proof. For notational simplicity, we drop the superscript (1) in the frequencies $n^{(1)}=n_{r}$ and $n_{j}^{(1)}$. In view of (2.4), the condition $0<\left|\phi_{1}\right| \leqslant K$ implies that

$$
\langle n\rangle^{-s}\left\langle n_{1}\right\rangle^{-s}\left\langle n_{2}\right\rangle^{-s}\left\langle n_{3}\right\rangle^{-s} \lesssim n_{\max }^{-4 s} \leqslant K^{-2 s}
$$

on $\Gamma(n)$, provided that $s \leqslant 0$. Then, by crudely estimating the contribution with Cauchy-Schwarz inequality, (8.7), and $|\mathfrak{B T}(1)|=1$, we have

$$
\begin{aligned}
& \sum_{n \in \mathbb{Z}}\left|\mathcal{N}_{1}^{(1)}(\mathbf{u})_{n}\right| \lesssim \sum_{n \in \mathbb{Z}} \sum_{\mathcal{T}_{1} \in \mathfrak{B} \mathfrak{T}(1)} \sum_{\substack { \phi \mid \leqslant K \\
\begin{subarray}{c}{\mathbf{n} \in \mathfrak{N}\left(\mathcal{T}_{1}\right) \\
n_{r}=n \\
\phi_{1}=\phi{ \phi | \leqslant K \\
\begin{subarray} { c } { \mathbf { n } \in \mathfrak { N } ( \mathcal { T } _ { 1 } ) \\
n _ { r } = n \\
\phi _ { 1 } = \phi } }\end{subarray}} \prod_{a \in \mathcal{T}_{1}^{\infty}}\left|\mathbf{u}_{n_{a}}\right| \\
& \lesssim\|\mathbf{u}\|_{H^{s}}\left\{\sup _{n \in \mathbb{Z}}\left(\sum_{\Gamma(n)} \frac{K^{1-4 s}}{n_{\max }^{2}\left|\left(n-n_{1}\right)\left(n-n_{3}\right)\right|}\right) \cdot\left(\sum_{n \in \mathbb{Z}} \sum_{\Gamma(n)} \prod_{i=1}^{3}\left\langle n_{i}\right\rangle^{2 s}\left|\mathbf{u}_{n_{i}}\right|^{2}\right)\right\}^{1 / 2} \\
& \lesssim K^{1 / 2-2 s}\|\mathbf{u}\|_{H^{s}}^{4} .
\end{aligned}
$$

This proves (8.6). Note that the power of $K$ is by no means sharp.

Next, we consider the nonresonant term $\mathcal{N}_{2}^{(1)}(\mathbf{u})$. It turns out that there is no effective estimate for $\mathcal{N}_{2}^{(1)}(\mathbf{u})$ and thus we perform a normal form reduction. In the following, we restrict our discussion to

$$
\left|\phi_{1}\right|>K
$$

namely, the set of frequencies are restricted onto $A_{K}^{c}$. When it is clear from the context, however, we suppress such restriction for notational simplicity. Differentiating by parts, that is, integrating by parts without an integral sign, we obtain

$$
\begin{aligned}
& \mathcal{N}_{2}^{(1)}(\mathbf{u})_{n}=2 \operatorname{Re} \partial_{t}\left[\sum_{\mathcal{T}_{1} \in \mathfrak{B} \mathfrak{T}(1)} \sum_{\substack{\mathbf{n} \in \mathfrak{N}\left(\mathcal{T}_{1}\right) \\
n_{r}=n}} \frac{e^{-i \phi_{1} t}}{\phi_{1}} \prod_{a \in \mathcal{T}_{1}^{\infty}} \mathbf{u}_{n_{a}}\right] \\
& -2 \operatorname{Re} \sum_{\mathcal{T}_{1} \in \mathfrak{B} \mathfrak{T}(1)} \sum_{\substack{n \in \mathfrak{N}\left(\mathcal{T}_{1}\right) \\
n_{r}=n}} \frac{e^{-i \phi_{1} t}}{\phi_{1}} \partial_{t}\left(\prod_{a \in \mathcal{T}_{1}^{\infty}} \mathbf{u}_{n_{a}}\right) \\
& =2 \operatorname{Re} \partial_{t}\left[\sum_{\mathcal{T}_{1} \in \mathfrak{B} \mathfrak{T}(1)} \sum_{\substack{\mathbf{n} \in \mathfrak{N}\left(\mathcal{T}_{1}\right) \\
n_{r}=n}} \frac{e^{-i \phi_{1} t}}{\phi_{1}} \prod_{a \in \mathcal{T}_{1}^{\infty}} \mathbf{u}_{n_{a}}\right] \\
& -2 \operatorname{Re} \sum_{\mathcal{T}_{1} \in \mathfrak{B} \mathfrak{T}(1)} \sum_{b \in \mathcal{T}_{1}^{\infty}} \sum_{\substack{\mathbf{n} \in \mathfrak{N}\left(\mathcal{T}_{1}\right) \\
n_{r}=n}} \frac{e^{-i \phi_{1} t}}{\phi_{1}} \mathrm{R}(\mathbf{u})_{n_{b}} \prod_{a \in \mathcal{T}_{1}^{\infty} \backslash\{b\}} \mathbf{u}_{n_{a}}
\end{aligned}
$$




$$
\begin{aligned}
& -2 \operatorname{Re} \sum_{\mathcal{T}_{2} \in \mathfrak{B} \mathfrak{T}(2)} \sum_{\substack{\mathbf{n} \in \mathfrak{N}\left(\mathcal{T}_{2}\right) \\
n_{r}=n}} \frac{e^{-i\left(\phi_{1}+\phi_{2}\right) t}}{\phi_{1}} \prod_{a \in \mathcal{T}_{2}^{\infty}} \mathbf{u}_{n_{a}} \\
& =: \partial_{t} \mathcal{N}_{0}^{(2)}(\mathbf{u})_{n}+\mathcal{R}^{(2)}(\mathbf{u})_{n}+\mathcal{N}^{(2)}(\mathbf{u})_{n} \text {. }
\end{aligned}
$$

In the second equality, we applied the product rule and used the equation (2.2) to replace $\partial_{t} \mathbf{u}_{n_{b}}$ by the resonant part $R(\mathbf{u})_{n_{b}}$ and the nonresonant part $\mathrm{N}(\mathbf{u})_{n_{b}}$. Note that substituting the nonresonant part $\mathrm{N}(\mathbf{u})_{n_{b}}$ amounts to extending the tree $\mathcal{T}_{1} \in$ $\mathfrak{B T}(1)$ (and $\mathbf{n} \in \mathfrak{N}\left(\mathcal{T}_{1}\right)$ ) to $\mathcal{T}_{2} \in \mathfrak{B T}(2)$ (and to $\mathbf{n} \in \mathfrak{N}\left(\mathcal{T}_{2}\right)$, respectively) by replacing the terminal node $b \in \mathcal{T}_{1}^{\infty}$ into a nonterminal node with three children $b_{1}, b_{2}$, and $b_{3}$.

REMARK 8.7. Strictly speaking, the phase factor appearing in $\mathcal{N}^{(2)}(\mathbf{u})$ may be $\phi_{1}-\phi_{2}$ when the time derivative falls on the terms with the complex conjugate. In the following, however, we simply write it as $\phi_{1}+\phi_{2}$ since it does not make any difference for our analysis. Also, we often replace \pm 1 and $\pm i$ by 1 for simplicity when they do not play an important role. Finally, for notational simplicity, we drop twice the real part symbol ' 2 Re' on multilinear forms, but it is understood that all the multilinear forms appear with twice the real part symbol.

We first estimate the boundary term $\mathcal{N}_{0}^{(2)}$.

LEMMA 8.8. Let $\mathcal{N}_{0}^{(2)}$ be as in (8.9). Then, for $s \geqslant-\frac{1}{2}$, we have

$$
\sum_{n \in \mathbb{Z}}\left|\mathcal{N}_{0}^{(2)}(\mathbf{u})_{n}\right| \lesssim K^{\max (-1 / 2,-1-2 s)}\|\mathbf{u}\|_{H^{s}}^{4}
$$

Proof. As in the proof of Lemma 8.5, we drop the superscript (1). From (2.4), we have

$$
\sup _{n \in \mathbb{Z}} \sum_{\substack{\Gamma(n) \\\left|\phi_{1}\right|>K}} \frac{n_{\max }^{-8 s}}{\left|\phi_{1}\right|^{2}} \lesssim \sup _{n \in \mathbb{Z}} \sum_{\substack{\Gamma(n) \\\left|\phi_{1}\right|>K}} \frac{1}{\left|\left(n-n_{1}\right)\left(n-n_{3}\right)\right|^{2} n_{\max }^{4+8 s}} \lesssim K^{\max (-1,-2-4 s)}
$$

for $s \geqslant-\frac{1}{2}$. Then, by Cauchy-Schwarz inequality with (8.11) and $|\mathfrak{B T}(1)|=1$, we have

$$
\sum_{n \in \mathbb{Z}}\left|\mathcal{N}_{0}^{(2)}(\mathbf{u})_{n}\right| \lesssim \sum_{\mathcal{T}_{1} \in \mathfrak{B} \mathfrak{T}(1)} \sum_{\substack { n \in \mathbb{Z} \\
\begin{subarray}{c}{n \in \mathfrak{N}\left(\mathcal{T}_{1}\right) \\
n_{r}=n \\
\text { i } 1>>K{ n \in \mathbb { Z } \\
\begin{subarray} { c } { n \in \mathfrak { N } ( \mathcal { T } _ { 1 } ) \\
n _ { r } = n \\
\text { i } 1 > > K } }\end{subarray}} \frac{n_{\max }^{-4 s}}{\left|\phi_{1}\right|} \prod_{a \in \mathcal{T}_{1}^{\infty}}\left\langle n_{a}\right\rangle^{s} \mathbf{u}_{n_{a}}
$$




$$
\begin{aligned}
& \leqslant\|\mathbf{u}\|_{H^{s}}\left\{\left(\sup _{n \in \mathbb{Z}} \sum_{\substack{\Gamma(n) \\
\left|\phi_{1}\right|>K}} \frac{n_{\max }^{-8 s}}{\left|\phi_{1}\right|^{2}}\right) \cdot\left(\sum_{n \in \mathbb{Z}} \sum_{\Gamma(n)} \prod_{i=1}^{3}\left\langle n_{i}\right\rangle^{2 s}\left|\mathbf{u}_{n_{i}}\right|^{2}\right)\right\}^{1 / 2} \\
& \lesssim K^{\max (-1 / 2,-1-2 s)}\|\mathbf{u}\|_{H^{s}}^{4} .
\end{aligned}
$$

This proves (8.10).

The following estimate on $\mathcal{R}^{(2)}$ is an immediate corollary to Lemma 8.8.

LEMMA 8.9. Let $\mathcal{R}^{(2)}$ be as in (8.9). Then, for $s \geqslant-\frac{1}{3}$, we have

$$
\sum_{n \in \mathbb{Z}}\left|\mathcal{R}^{(2)}(\mathbf{u})_{n}\right| \lesssim K^{\max (-1 / 2,-1-3 s)}\|\mathbf{u}\|_{H^{s}}^{6}
$$

Proof. This lemma follows from the proof of Lemma 8.8 and $\ell^{2} \subset \ell^{6}$ once we observe that

$$
\sum_{\substack{\Gamma(n) \\\left|\phi_{1}\right|>K}} \frac{n_{\max }^{-12 s}}{\left|\phi_{1}\right|^{2}} \lesssim \sum_{\substack{\Gamma(n) \\\left|\phi_{1}\right|>K}} \frac{1}{\left|\mu_{1}\right|^{2}\left\langle n_{\max }\right\rangle^{4+12 s}} \lesssim K^{\max (-1,-2-6 s)}
$$

provided that $s \geqslant-\frac{1}{3}$.

As in the first step of the normal form reductions, we cannot estimate $\mathcal{N}^{(2)}$ as it is. By dividing the frequency space into

$$
C_{1}=\left\{\left|\phi_{1}+\phi_{2}\right| \lesssim 6^{3}\left|\phi_{1}\right|^{1-\theta}\right\}
$$

for some $\theta \in(0,1)$ (to be chosen later) and its complement $C_{1}^{c}$, split $\mathcal{N}^{(2)}$ as

$$
\mathcal{N}^{(2)}=\mathcal{N}_{1}^{(2)}+\mathcal{N}_{2}^{(2)}
$$

where $\mathcal{N}_{1}^{(2)}$ is the restriction of $\mathcal{N}^{(2)}$ onto $C_{1}$ and $\mathcal{N}_{2}^{(2)}:=\mathcal{N}^{(2)}-\mathcal{N}_{1}^{(2)}$. (Clearly, the number $6^{3}$ in (8.12) does not make any difference at this point. However, we insert it to match with (8.30). See also (8.16).)

Thanks to the frequency restriction, we can estimate the first term $\mathcal{N}_{1}^{(2)}$ as follows.

LEMMA 8.10. Let $\mathcal{N}_{1}^{(2)}$ be as in (8.13). Then, for $s>-\frac{1}{3}$, we have

$$
\sum_{n \in \mathbb{Z}}\left|\mathcal{N}_{1}^{(2)}(\mathbf{u})_{n}\right| \lesssim\|\mathbf{u}\|_{H^{s}}^{6}
$$


Before presenting the proof of Lemma 8.10 , let us briefly describe how to handle the second term of $\mathcal{N}_{2}^{(2)}$. On the support of $\mathcal{N}_{2}^{(2)}$, we have

$$
\left|\phi_{1}+\phi_{2}\right| \gg 6^{3}\left|\phi_{1}\right|^{1-\theta}>6^{3} K^{1-\theta} \text {. }
$$

Namely, the phase function $\phi_{1}+\phi_{2}$ is 'large' in this case and hence we can exploit this fast oscillation by applying the second step of the normal form reduction:

$$
\begin{aligned}
\mathcal{N}_{2}^{(2)}(\mathbf{u})_{n}= & \partial_{t}\left[\sum_{\mathcal{T}_{2} \in \mathfrak{B} \mathfrak{T}(2)} \sum_{\substack{\mathbf{n} \in \mathfrak{N}\left(\mathcal{T}_{2}\right) \\
n_{r}=n}} \frac{e^{-i\left(\phi_{1}+\phi_{2}\right) t}}{\phi_{1}\left(\phi_{1}+\phi_{2}\right)} \prod_{a \in \mathcal{T}_{2}^{\infty}} \mathbf{u}_{n_{a}}\right] \\
& -\sum_{\mathcal{T}_{2} \in \mathfrak{B} \mathfrak{T}(2)} \sum_{\substack{\mathbf{n} \in \mathfrak{N}\left(\mathcal{T}_{n}\right) \\
n_{r}=n}} \frac{e^{-i\left(\phi_{1}+\phi_{2}\right) t}}{\phi_{1}\left(\phi_{1}+\phi_{2}\right)} \partial_{t}\left(\prod_{a \in \mathcal{T}_{2}^{\infty}} \mathbf{u}_{n_{a}}\right) \\
= & \partial_{t}\left[\sum_{\mathcal{T}_{2} \in \mathfrak{B} \mathfrak{T}(2)} \sum_{\mathbf{n} \in \mathfrak{N}\left(\mathcal{T}_{2}\right)} \frac{e^{-i\left(\phi_{1}+\phi_{2}\right) t}}{\phi_{1}\left(\phi_{1}+\phi_{2}\right)} \prod_{a \in \mathcal{T}_{2}^{\infty}} \mathbf{u}_{n_{a}}\right] \\
& -\sum_{\mathcal{T}_{2} \in \mathfrak{B} \mathfrak{T}(2)} \sum_{\substack{b \in \mathcal{T}_{2}^{\infty} \\
\mathbf{n} \in \mathfrak{n}_{r}=n}} \frac{e^{-i\left(\mathcal{T}_{2}\right)}}{\phi_{1}\left(\phi_{1}\right) t} \phi_{\left.\phi_{2}\right)} \mathrm{R}(\mathbf{u})_{n_{b}} \prod_{a \in \mathcal{T}_{2}^{\infty} \backslash\{b\}} \mathbf{u}_{n_{a}} \\
& -\sum_{\mathcal{T}_{3} \in \mathfrak{B} \mathfrak{T}(3)} \sum_{\substack{\mathbf{n} \in \mathfrak{N}\left(\mathcal{T}_{3}\right) \\
n_{r}=n}} \frac{e^{-i\left(\phi_{1}+\phi_{2}+\phi_{3}\right) t}}{\phi_{1}\left(\phi_{1}+\phi_{2}\right)} \prod_{a \in \mathcal{T}_{3}^{\infty}} \mathbf{u}_{n_{a}} \\
= & : \partial_{t} \mathcal{N}_{0}^{(3)}(\mathbf{u})_{n}+\mathcal{R}^{(3)}(\mathbf{u})_{n}+\mathcal{N}^{(3)}(\mathbf{u})_{n} .
\end{aligned}
$$

The first two terms $\mathcal{N}_{0}^{(3)}$ and $\mathcal{R}^{(3)}$ on the right-hand side can be estimated in a straightforward manner with (8.8) and (8.14). See Lemmas 8.12 and 8.13 below. As for the last term $\mathcal{N}^{(3)}$, we split it as

$$
\mathcal{N}^{(3)}=\mathcal{N}_{1}^{(3)}+\mathcal{N}_{2}^{(3)},
$$

where $\mathcal{N}_{1}^{(3)}$ and $\mathcal{N}_{2}^{(3)}$ are the restrictions onto

$$
C_{2}=\left\{\left|\phi_{1}+\phi_{2}+\phi_{3}\right| \lesssim 8^{3}\left|\phi_{1}+\phi_{2}\right|^{1-\theta}\right\} \cup\left\{\left|\phi_{1}+\phi_{2}+\phi_{3}\right| \lesssim 8^{3}\left|\phi_{1}\right|^{1-\theta}\right\}
$$

and its complement $C_{2}^{c}$, respectively. By exploiting the frequency restriction, we can estimate the first term $\mathcal{N}_{1}^{(3)}$ (see Lemma 8.14 below). As for the second term $\mathcal{N}_{2}^{(3)}$, we apply the third step of the normal form reductions. In this way, we iterate normal form reductions in an indefinite manner.

We conclude this subsection by presenting the proof of Lemma 8.10. 
Proof of Lemma 8.10. Note that we have $\left|\phi_{2}\right| \sim\left|\phi_{1}\right|$ thanks to (8.12). Then, with (8.4) and $\left|\mu_{j}\right| \lesssim\left(n_{\max }^{(j)}\right)^{2}, j=1,2$, we have

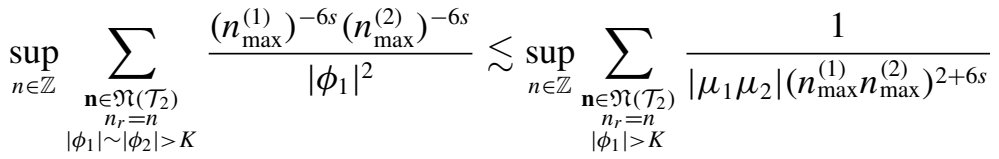

$$
\begin{aligned}
& \lesssim \sup _{n \in \mathbb{Z}} \sum_{\substack{\mathbf{n} \in \mathfrak{N}\left(\mathcal{T}_{2}\right) \\
n_{r}=n}} \frac{1}{\left|\mu_{1} \mu_{2}\right|^{1+}} \lesssim 1
\end{aligned}
$$

provided that $s>-\frac{1}{3}$. In the last step, we first summed over $n_{1}^{(2)}$ and $n_{3}^{(2)}$ for fixed $n^{(2)}$ and then summed over $n_{1}^{(1)}$ and $n_{3}^{(1)}$ for fixed $n$.

- Case 1: We first consider the case $\Pi_{2}\left(\mathcal{T}_{2}\right)=\left\{r_{2}\right\}$. Namely, the second root node $r_{2}$ is a terminal node. By Cauchy-Schwarz inequality with (8.17), we have

$$
\begin{aligned}
& \sum_{n \in \mathbb{Z}}\left|\mathcal{N}_{1}^{(2)}(\mathbf{u})_{n}\right| \lesssim \sum_{n \in \mathbb{Z}} \sum_{\substack{\mathcal{T}_{2} \in \mathfrak{B} \mathfrak{T}(2) \\
\Pi_{2}\left(\mathcal{T}_{2}\right)=\left\{r_{2}\right\}}} \sum_{\substack{\mathbf{n} \in \mathfrak{N}\left(\mathcal{T}_{2}\right) \\
n_{r}=n \\
\left|\phi_{1}\right|>K}} \frac{1}{\left|\phi_{1}\right|} \prod_{\substack{a \in \mathcal{T}_{2}^{\infty} \\
\mathbf{u}_{n_{a}}}} \mathbf{u}
\end{aligned}
$$

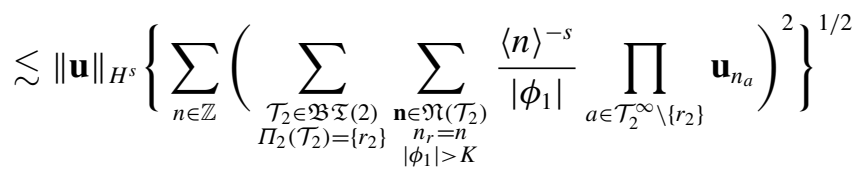

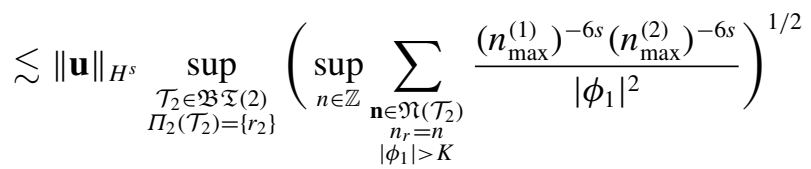

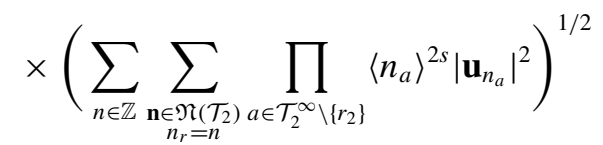

$$
\begin{aligned}
& \lesssim\|\mathbf{u}\|_{H^{s}}^{6} .
\end{aligned}
$$

In the last step, we used the observations in Remark 8.4.

- Case 2: Next, we consider the case $\Pi_{2}\left(\mathcal{T}_{2}\right) \neq\left\{r_{2}\right\}$. In this case, we need to modify the argument above since the frequency $n_{r}=n$ does not correspond to a terminal node. Noting that $\mathcal{T}_{2}^{\infty}=\Pi_{1}\left(\mathcal{T}_{2}\right)^{\infty} \cup \Pi_{2}\left(\mathcal{T}_{2}\right)^{\infty}$ and hence

$$
\sum_{\substack{\mathbf{n} \in \mathfrak{N}\left(\mathcal{T}_{2}\right) \\ n_{r}=n}} \prod_{a \in \mathcal{T}_{2}^{\infty}}\left|\mathbf{u}_{n_{a}}\right|^{2}=\prod_{j=1}^{2}\left(\sum_{\substack{\mathbf{n} \in \mathfrak{N}\left(\Pi_{j}\left(\mathcal{T}_{2}\right)\right) \\ n_{r_{j}}=n}} \prod_{a_{j} \in \Pi_{j}\left(\mathcal{T}_{2}\right)^{\infty}}\left|\mathbf{u}_{n_{a_{j}}}\right|^{2}\right),
$$


we have

$$
\begin{aligned}
& \sum_{n \in \mathbb{Z}}\left|\mathcal{N}_{1}^{(2)}(\mathbf{u})_{n}\right| \lesssim \sum_{n \in \mathbb{Z}} \sum_{\substack{\mathcal{T}_{2} \in \mathfrak{B} \mathfrak{T}(2) \\
\Pi_{2}\left(\mathcal{T}_{2}\right) \neq\left\{r_{2}\right\}}} \sum_{\substack{\mathbf{n} \in \mathfrak{N}\left(\mathcal{T}_{2}\right) \\
n_{r}=n \\
\left|\phi_{1}\right|>K}} \frac{1}{\left|\phi_{1}\right|} \prod_{a \in \mathcal{T}_{2}^{\infty}} \mathbf{u}_{n_{a}} \\
& \lesssim \sup _{\substack{\mathcal{T}_{2} \in \mathfrak{B} \mathfrak{T}(2) \\
\Pi_{2}\left(\mathcal{T}_{2}\right) \neq\left\{r_{2}\right\}}} \sum_{n \in \mathbb{Z}}\left(\sum_{\substack{\mathbf{n} \in \mathfrak{N}\left(\mathcal{T}_{2}\right) \\
n_{r}=n \\
\phi_{1} \mid>K}} \frac{\left(n_{\max }^{(1)}\right)^{-6 s}\left(n_{\max }^{(2)}\right)^{-6 s}}{\left|\phi_{1}\right|^{2}}\right)^{1 / 2}\left(\sum_{\substack{\mathbf{n} \in \mathfrak{N}\left(\mathcal{T}_{2}\right) \\
n_{r}=n}} \prod_{a \in \mathcal{T}_{2}^{\infty}}\left\langle n_{a}\right\rangle^{2 s}\left|\mathbf{u}_{n_{a}}\right|^{2}\right)^{1 / 2} \\
& \lesssim \sup _{\substack{\mathcal{T}_{2} \in \mathfrak{B} \mathfrak{T}(2) \\
\Pi_{2}\left(\mathcal{T}_{2}\right) \neq\left\{r_{2}\right\}}} \sum_{n \in \mathbb{Z}}\left(\sum_{\substack{\mathbf{n} \in \mathfrak{N}\left(\mathcal{T}_{2}\right) \\
n_{r}=n}} \prod_{a \in \mathcal{T}_{2}^{\infty}}\left\langle n_{a}\right\rangle^{2 s}\left|\mathbf{u}_{n_{a}}\right|^{2}\right)^{1 / 2} \\
& \lesssim \sup _{\substack{\mathcal{T}_{2} \in \mathfrak{B} \mathfrak{T}(2) \\
\Pi_{2}\left(\mathcal{T}_{2}\right) \neq\left\{r_{2}\right\}}} \sum_{n \in \mathbb{Z}} \prod_{j=1}^{2}\left(\sum_{\substack{\mathbf{n} \in \mathfrak{N}\left(\Pi_{j}\left(\mathcal{T}_{2}\right)\right) \\
n_{r_{j}}=n}} \prod_{a_{j} \in \Pi_{j}\left(\mathcal{T}_{2}\right)^{\infty}}\left\langle n_{a_{j}}\right\rangle^{2 s}\left|\mathbf{u}_{n_{a_{j}}}\right|^{2}\right)^{1 / 2}
\end{aligned}
$$

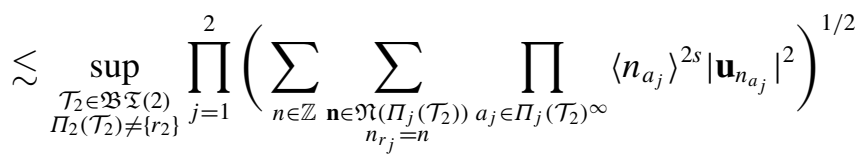

$$
\begin{aligned}
& \lesssim\|\mathbf{u}\|_{H^{s}}^{6} .
\end{aligned}
$$

This completes the proof of Lemma 8.10.

REMARK 8.11. The above computation in Cases 1 and 2 in particular shows that, given $\mathcal{T}_{j} \in \mathfrak{B T}(j), j \in \mathbb{N}$, we have

$$
\sum_{n \in \mathbb{Z}}\left(\sum_{\substack{\mathbf{n} \in \mathfrak{N}\left(\mathcal{T}_{j}\right) \\ n_{r}=n}} \prod_{a \in \mathcal{T}_{j}^{\infty}}\left|\mathbf{u}_{n_{a}}\right|^{2}\right)^{1 / 2} \leqslant\left\|\mathbf{u}_{n}\right\|_{\ell_{n}^{2}}^{2 j+2}
$$

8.3. General step: $\boldsymbol{J}$ th generation. After the $J$ th step, we have

$$
\begin{aligned}
& \mathcal{N}_{2}^{(J)}(\mathbf{u})_{n}=\partial_{t}\left[\sum_{\mathcal{T} J \in \mathfrak{B} \mathfrak{T}(J)} \sum_{\substack{\mathbf{n} \in \mathfrak{N}\left(\mathcal{T}_{J}\right) \\
n_{r}=n}} \frac{e^{-i \widetilde{\phi}_{J} t}}{\prod_{j=1}^{J} \widetilde{\phi}_{j}} \prod_{a \in \mathcal{T}_{J}^{\infty}} \mathbf{u}_{n_{a}}\right]
\end{aligned}
$$

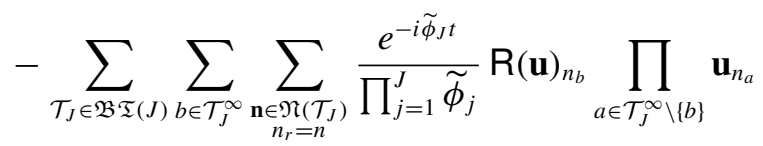




$$
\begin{gathered}
-\sum_{\mathcal{T}_{J+1} \in \mathfrak{B} \mathfrak{T}(J+1)} \sum_{\substack{\mathbf{n} \in \mathfrak{N}\left(\mathcal{T}_{J+1}\right) \\
n_{r}=n}} \frac{e^{-i \tilde{\phi}_{J+1} t}}{\prod_{j=1}^{J} \widetilde{\phi}_{j}} \prod_{a \in \mathcal{T}_{J+1}^{\infty}} \mathbf{u}_{n_{a}} \\
=: \partial_{t} \mathcal{N}_{0}^{(J+1)}(\mathbf{u})_{n}+\mathcal{R}^{(J+1)}(\mathbf{u})_{n}+\mathcal{N}^{(J+1)}(\mathbf{u})_{n},
\end{gathered}
$$

where $\widetilde{\phi}_{J}$ is defined by

$$
\widetilde{\phi}_{J}:=\sum_{j=1}^{J} \phi_{j}
$$

Recall that $\left|\phi_{1}\right|>K$ and

$$
\left|\widetilde{\phi}_{j}\right| \gg(2 j+2)^{3} \max \left(\left|\widetilde{\phi}_{j-1}\right|^{1-\theta},\left|\phi_{1}\right|^{1-\theta}\right)>(2 j+2)^{3} K^{1-\theta},
$$

for $j=2, \ldots, J$. One of the main tasks in estimating the multilinear forms in (8.18) is to control the rapidly growing cardinality $c_{J}=|\mathfrak{B T}(J)|$ defined in (8.2). As in [18], we control $c_{J}$ by the growing constant $(2 j+2)^{3}$ appearing in (8.20). First, we estimate $\mathcal{N}_{0}^{(J+1)}$ and $\mathcal{R}^{(J+1)}$.

LEMMA 8.12. Let $\mathcal{N}_{0}^{(J+1)}$ be as in (8.18). Then, for $s>-\frac{1}{3}$, we have

$$
\sum_{n \in \mathbb{Z}}\left|\mathcal{N}_{0}^{(J+1)}(\mathbf{u})_{n}\right| \lesssim K^{-J(1-\theta) / 2}\|\mathbf{u}\|_{H^{s}}^{2 J+2}
$$

The implicit constant is independent of $J$. The same comment applies to Lemmas 8.13 and 8.14 below.

Proof. From (8.19), we have

$$
\left|\phi_{j}\right| \lesssim \max \left(\left|\widetilde{\phi}_{j-1}\right|,\left|\widetilde{\phi}_{j}\right|\right)
$$

Then, in view of (8.20), we have

$$
(2 j)^{3} K^{1-\theta}\left|\phi_{j}\right| \ll\left|\widetilde{\phi}_{j-1}\right|\left|\widetilde{\phi}_{j}\right| .
$$

Hence, with (8.20) once again, we have

$$
\prod_{j=1}^{J}\left((2 j+2)^{3} K^{1-\theta}\left|\phi_{j}\right|\right) \ll\left|\phi_{1}\right|\left|\widetilde{\phi}_{J}\right| \prod_{j=2}^{J}\left((2 j)^{3} K^{1-\theta}\left|\phi_{j}\right|\right) \ll \prod_{j=1}^{J}\left|\widetilde{\phi}_{j}\right|^{2} .
$$

We only discuss the case $\Pi_{2}\left(\mathcal{T}_{J}\right)=\left\{r_{2}\right\}$ since the modification is straightforward if $\Pi_{2}\left(\mathcal{T}_{J}\right) \neq\left\{r_{2}\right\}$. As in (8.17), we have

$$
\frac{\left(n_{\max }^{(j)}\right)^{-6 s}}{\left|\phi_{j}\right|} \sim \frac{\left(n_{\max }^{(j)}\right)^{-6 s}}{\left|\mu_{j}\right|\left(n_{\max }^{(j)}\right)^{2}} \lesssim \frac{1}{\left|\mu_{j}\right|^{1+}}
$$


for $s>-\frac{1}{3}$. Then, by (8.22) and (8.23), we have

$$
\begin{aligned}
& \sup _{n \in \mathbb{Z}} \sum_{\substack{n \\
\mathbf{n} \in \mathfrak{N}\left(\mathcal{T}_{J}\right) \\
n_{r}=n \\
\left|\phi_{1}\right|>K \\
\left|\widetilde{\phi}_{j}\right| \gg(2 j+2)^{3} K^{1-\theta} \\
j=2, \ldots, J}} \prod_{j=1}^{J} \frac{\left(n_{\max }^{(j)}\right)^{-6 s}}{\left|\widetilde{\phi}_{j}\right|^{2}} \ll \frac{K^{-J(1-\theta)}}{\prod_{j=1}^{J}(2 j+2)^{3}} \cdot \sup _{\substack { n \in \mathbb{Z} \\
\begin{subarray}{c}{n \in \mathfrak{N}\left(\mathcal{T}_{J}\right) \\
n_{r}=n \\
\phi_{j} \neq 0 \\
j=1, \ldots, J{ n \in \mathbb { Z } \\
\begin{subarray} { c } { n \in \mathfrak { N } ( \mathcal { T } _ { J } ) \\
n _ { r } = n \\
\phi _ { j } \neq 0 \\
j = 1 , \ldots , J } }\end{subarray}} \prod_{j=1}^{J} \frac{\left(n_{\max }^{(j)}\right)^{-6 s}}{\left|\phi_{j}\right|} \\
& \lesssim \frac{K^{-J(1-\theta)}}{\prod_{j=1}^{J}(2 j+2)^{3}} \cdot \sup _{\substack { n \in \mathbb{Z} \\
\begin{subarray}{c}{\mathbf{n} \in \mathfrak{N}\left(\mathcal{T}_{J}\right) \\
n_{r}=n \\
\mu_{j} \neq 0 \\
j=1, \ldots, J{ n \in \mathbb { Z } \\
\begin{subarray} { c } { \mathbf { n } \in \mathfrak { N } ( \mathcal { T } _ { J } ) \\
n _ { r } = n \\
\mu _ { j } \neq 0 \\
j = 1 , \ldots , J } }\end{subarray}} \prod_{j=1}^{J} \frac{1}{\left|\mu_{j}\right|^{1+}} \\
& \leqslant \frac{C^{J} K^{-J(1-\theta)}}{\prod_{j=1}^{J}(2 j+2)^{3}} .
\end{aligned}
$$

By Cauchy-Schwarz inequality with (8.8), (8.20), and (8.24), we have

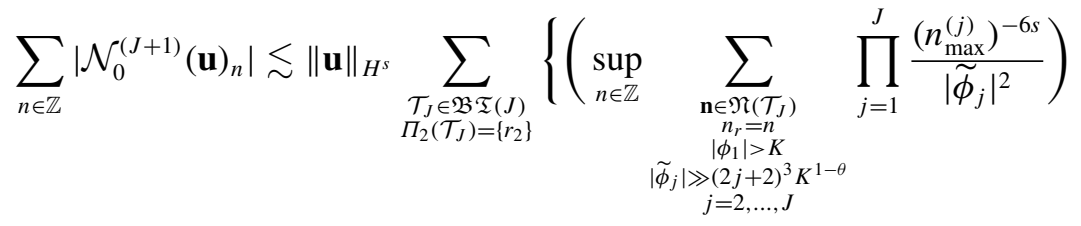

$$
\begin{aligned}
& \left.\times\left(\sum_{n \in \mathbb{Z}} \sum_{\substack{\mathbf{n} \in \mathfrak{N}\left(\mathcal{T}_{J}\right) \\
n_{r}=n}} \prod_{\substack{a \in \mathcal{T}_{J}^{\infty} \backslash\left\{r_{2}\right\} \\
C^{J}}}\left\langle n_{a}\right\rangle^{2 s}\left|\mathbf{u}_{n_{a}}\right|^{2}\right)\right\}^{1 / 2} \\
& \lesssim \frac{c_{J} \cdot C^{J / 2}}{\prod_{j=1}^{J}(2 j+2)^{3 / 2}} K^{-J(1-\theta) / 2}\|\mathbf{u}\|_{H^{s}}^{2 J+2} \lesssim K^{-J(1-\theta) / 2}\|\mathbf{u}\|_{H^{s}}^{2 J+2} .
\end{aligned}
$$

This completes the proof of Lemma 8.12.

LEMMA 8.13. Let $\mathcal{R}^{(J+1)}$ be as in (8.18). Then, for $s \geqslant \max \left(-\frac{1}{3}+,-(3-2 \theta) / 5\right)$, we have

$$
\sum_{n \in \mathbb{Z}}\left|\mathcal{R}^{(J+1)}(\mathbf{u})_{n}\right| \lesssim K^{-((J-2)(1-\theta)) / 2}\|\mathbf{u}\|_{H^{s}}^{2 J+4} .
$$

In particular, if $\theta \in\left(0, \frac{2}{3}\right]$, then (8.26) holds for $s>-\frac{1}{3}$.

Proof. Just like Lemma 8.9 on $\mathcal{R}^{(2)}$, this lemma follows from a modification of the proof of Lemma 8.12. 
We first consider the case $\left|\widetilde{\phi}_{J}\right| \gtrsim\left|\phi_{J}\right|$. Noting that $\left(n_{\max }^{(J)}\right)^{-4 s} \lesssim\left|\widetilde{\phi}_{J}\right|$ for $s \geqslant-\frac{1}{2}$, it follows from the second inequality in (8.22) and (8.23), we have

$$
\begin{aligned}
\sup _{n \in \mathbb{Z}} & \sum_{\substack{\mathbf{n} \in \mathfrak{N}\left(\mathcal{T}_{J}\right) \\
n_{r}=n \\
\left|\phi_{1}>K \\
\widetilde{\phi}_{j}\right| \gg(2 j+2)^{3} K^{1-\theta} \\
j=2, \ldots, J}}\left(n_{\max }^{(J)}\right)^{-4 s} \prod_{j=1}^{J} \frac{\left(n_{\max }^{(j)}\right)^{-6 s}}{\left|\widetilde{\phi}_{j}\right|^{2}} \\
& \ll \frac{K^{-(J-1)(1-\theta)}}{\prod_{j=2}^{J}(2 j+2)^{3}} \cdot \sup _{\substack { n \in \mathbb{Z} \\
\begin{subarray}{c}{\mathbf{n} \in \mathfrak{N}\left(\mathcal{T}_{J}\right) \\
n_{r}=n \\
\phi_{j} \neq 0 \\
j=1, \ldots, J{ n \in \mathbb { Z } \\
\begin{subarray} { c } { \mathbf { n } \in \mathfrak { N } ( \mathcal { T } _ { J } ) \\
n _ { r } = n \\
\phi _ { j } \neq 0 \\
j = 1 , \ldots , J } }\end{subarray}} \frac{\left(n_{\max }^{(J)}\right)^{-4 s}}{\left|\widetilde{\phi}_{J}\right|} \prod_{j=1}^{J} \frac{\left(n_{\max }^{(j)}\right)^{-6 s}}{\left|\phi_{j}\right|} \\
& \lesssim \frac{C^{J} K^{-(J-1)(1-\theta)}}{\prod_{j=2}^{J}(2 j+2)^{3}},
\end{aligned}
$$

provided that $s>-\frac{1}{3}$. Then, proceeding as in (8.25) with (8.27), we obtain (8.26) in this case.

Next, consider the case $\left|\widetilde{\phi}_{J}\right| \ll\left|\phi_{J}\right|$. In this case, we have $\left|\phi_{J}\right| \sim\left|\widetilde{\phi}_{J-1}\right|$. Proceeding as in (8.22) with (8.21), we have

$$
\left|\phi_{1}\right|\left|\widetilde{\phi}_{J-1}\right|\left|\widetilde{\phi}_{J}\right|^{2} \prod_{j=2}^{J-1}\left((2 j)^{3} K^{1-\theta}\left|\phi_{j}\right|\right) \ll \prod_{j=1}^{J}\left|\widetilde{\phi}_{j}\right|^{2} .
$$

From (8.20), we have $\left|\widetilde{\phi}_{J}\right| \gg(2 J+2)^{3}\left|\widetilde{\phi}_{J-1}\right|^{1-\theta} \sim(2 J+2)^{3}\left|\phi_{J}\right|^{1-\theta}$. This gives

$$
K^{(J-2)(1-\theta)}\left|\phi_{J}\right|^{2-2 \theta} \prod_{j=1}^{J}\left((2 j+2)^{3}\left|\phi_{j}\right|\right) \ll \prod_{j=1}^{J}\left|\widetilde{\phi}_{j}\right|^{2} .
$$

Hence, we obtain

$$
\begin{aligned}
\sup _{n \in \mathbb{Z}} & \sum_{\substack{n \in \mathfrak{N}\left(\mathcal{T}_{J}\right) \\
n_{r}=n \\
\left|\phi_{1}\right|>K \\
\left|\tilde{\phi}_{j}\right| \gg(2 j+2)^{3} K^{1-\theta} \\
j=2, \ldots, J}}\left(n_{\max }^{(J)}\right)^{-4 s} \prod_{j=1}^{J} \frac{\left(n_{\max }^{(j)}\right)^{-6 s}}{\left|\widetilde{\phi}_{j}\right|^{2}} \\
& \ll \frac{K^{-(J-2)(1-\theta)}}{\prod_{j=1}^{J}(2 j+2)^{3}} \cdot \sup _{\substack { n \in \mathbb{Z} \\
\begin{subarray}{c}{n \in \mathcal{N}\left(\mathcal{T}_{J}\right) \\
n_{r}=n \\
\phi_{j} \neq 0 \\
j=1, \ldots, J{ n \in \mathbb { Z } \\
\begin{subarray} { c } { n \in \mathcal { N } ( \mathcal { T } _ { J } ) \\
n _ { r } = n \\
\phi _ { j } \neq 0 \\
j = 1 , \ldots , J } }\end{subarray}} \frac{\left(n_{\max }^{(J)}\right)^{-10 s}}{\left|\phi_{J}\right|^{3-2 \theta}} \prod_{j=1}^{J-1} \frac{\left(n_{\max }^{(j)}\right)^{-6 s}}{\left|\phi_{j}\right|}
\end{aligned}
$$




$$
\lesssim \frac{C^{J} K^{-(J-2)(1-\theta)}}{\prod_{j=1}^{J}(2 j+2)^{3}}
$$

provided that $s>-\frac{1}{3}$ and $s \geqslant-(3-2 \theta) / 5$. Then, proceeding as in (8.25) with (8.28), we obtain (8.26) in this case.

Finally, we consider $\mathcal{N}^{(J+1)}$. As before, we write

$$
\mathcal{N}^{(J+1)}=\mathcal{N}_{1}^{(J+1)}+\mathcal{N}_{2}^{(J+1)}
$$

where $\mathcal{N}_{1}^{(J+1)}$ is the restriction of $\mathcal{N}^{(J+1)}$ onto

$$
C_{J}=\left\{\left|\widetilde{\phi}_{J+1}\right| \lesssim(2 J+2)^{3}\left|\widetilde{\phi}_{J}\right|^{1-\theta}\right\} \cup\left\{\left|\widetilde{\phi}_{J+1}\right| \lesssim(2 J+2)^{3}\left|\phi_{1}\right|^{1-\theta}\right\}
$$

and $\mathcal{N}_{2}^{(J+1)}:=\mathcal{N}^{(J+1)}-\mathcal{N}_{1}^{(J+1)}$. In the following lemma, we estimate the first term $\mathcal{N}_{1}^{(J+1)}$. Then, we apply a normal form reduction once again to the second term $\mathcal{N}_{2}^{(J+1)}$ as in (8.18) and repeat this process indefinitely. In the next subsection, we also show that this error term $\mathcal{N}_{2}^{(J+1)}$ tends to 0 in the $\ell_{n}^{1}$-nom as $J \rightarrow \infty$.

LEMMA 8.14. Let $\mathcal{N}_{1}^{(J+1)}$ be as in (8.29). Then, for $s>-\frac{1}{3}$, we have

$$
\sum_{n \in \mathbb{Z}}\left|\mathcal{N}_{1}^{(J+1)}(\mathbf{u})_{n}\right| \lesssim K^{-((J-1)(1-\theta)) / 2}\|\mathbf{u}\|_{H^{s}}^{2 J+4}
$$

Proof. We proceed with (8.21) as in the proofs of Lemmas 8.12 and 8.13. It follows from the restriction $\left|\widetilde{\phi}_{J+1}\right|=\left|\widetilde{\phi}_{J}+\phi_{J+1}\right| \lesssim(2 J+2)^{3}\left|\widetilde{\phi}_{J}\right|^{1-\theta}$ that $\left|\phi_{J+1}\right| \lesssim J^{3}\left|\widetilde{\phi}_{J}\right|$. Then from (8.21), we have

$$
\left|\phi_{1}\right|\left|\phi_{J+1}\right| \prod_{j=2}^{J}(2 j+2)^{3} K^{1-\theta}\left|\phi_{j}\right| \ll J^{3} \prod_{j=1}^{J}\left|\widetilde{\phi}_{j}\right|^{2} .
$$

Proceeding as in (8.24), we have

$$
\begin{aligned}
\sup _{n \in \mathbb{Z}} \sum_{\substack{\mathbf{n} \in \mathfrak{N}\left(\mathcal{T}_{J+1}\right) \\
n_{r}=n \\
\left|\phi_{1}\right|>K \\
\left(\tilde{\phi}_{j} \mid \gg j+2\right)^{3} K^{1-\theta} \\
j=2, \ldots, J}}\left(n_{\max }^{(J+1)}\right)^{-6 s} \prod_{j=1}^{J} \frac{\left(n_{\max }^{(j)}\right)^{-6 s}}{\left|\widetilde{\phi}_{j}\right|^{2}} \\
\ll \frac{K^{-(J-1)(1-\theta)}}{\prod_{j=2}^{J-1}(2 j+2)^{3}} \cdot \sup _{\substack { n \in \mathbb{Z} \\
\begin{subarray}{c}{\mathbf{n} \in \mathfrak{N}^{\prime}\left(\mathcal{T}_{J+1}\right) \\
n_{r}=n \\
\phi_{j} \neq \neq 0 \\
j=1, \ldots, J+1{ n \in \mathbb { Z } \\
\begin{subarray} { c } { \mathbf { n } \in \mathfrak { N } ^ { \prime } ( \mathcal { T } _ { J + 1 } ) \\
n _ { r } = n \\
\phi _ { j } \neq \neq 0 \\
j = 1 , \ldots , J + 1 } }\end{subarray}} \prod_{j=1}^{J+1} \frac{\left(n_{\max }^{(j)}\right)^{-6 s}}{\left|\phi_{j}\right|}
\end{aligned}
$$




$$
\lesssim \frac{C^{J+1} K^{-(J-1)(1-\theta)}}{\prod_{j=2}^{J-1}(2 j+2)^{3}}
$$

provided that $s>-\frac{1}{3}$. Then, (8.31) follows from the Cauchy-Schwarz argument with (8.32) once we note that

$$
\frac{c_{J+1} C^{(J+1) / 2}}{\prod_{j=2}^{J-1}(2 j+2)^{3 / 2}} \lesssim 1
$$

uniformly in $J$.

8.4. On the error term. In this subsection, we prove that the error term $\mathcal{N}_{2}^{(J+1)}(\mathbf{u})$ tends to 0 as $J \rightarrow \infty$ under some regularity assumption on $\mathbf{u}$.

From (8.18), we have

$$
\begin{aligned}
& \mathcal{N}_{2}^{(J+1)}(\mathbf{u})_{n}=-\sum_{\mathcal{T}_{J+1} \in \mathfrak{B} \mathfrak{T}(J+1)} \sum_{\substack{\mathbf{n} \in \mathfrak{N}\left(\mathcal{T}_{J+1}\right) \\
n_{r}=n}} \frac{e^{-i \widetilde{\phi}_{J+1} t}}{\prod_{j=1}^{J} \widetilde{\phi}_{j}} \prod_{a \in \mathcal{T}_{J+1}^{\infty}} \mathbf{u}_{n_{a}}
\end{aligned}
$$

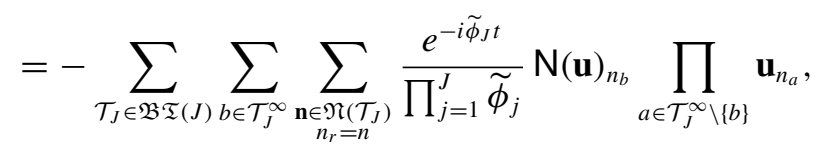

where it is understood that the summations in (8.33) are restricted to frequencies satisfying (8.8) and (8.20). (In fact, $\mathcal{N}_{2}^{(J+1)}$ is also restricted to $C_{J}^{c}$ but we do not need to use this fact. Namely, our argument also shows that $\mathcal{N}^{(J+1)}(\mathbf{u}) \rightarrow 0$ as $J \rightarrow \infty$.)

LEMMA 8.15. Let $\mathcal{N}_{2}^{(J+1)}$ be as in (8.33). Then, given $\mathbf{u} \in H^{1 / 6}(\mathbb{T})$, we have

$$
\sum_{n \in \mathbb{Z}}\left|\mathcal{N}_{2}^{(J+1)}(\mathbf{u})_{n}\right| \longrightarrow 0
$$

as $J \rightarrow \infty$.

Proof. The following simple estimate yields the minimum regularity restriction $s \geqslant \frac{1}{6}$, required for $\mathcal{N}_{2}^{(J+1)} \rightarrow 0$ as $J \rightarrow \infty$. By Hausdorff-Young's, Hölder's, and Sobolev's inequalities, we have

$$
\left\|\mathrm{N}(\mathbf{u})_{n_{b}}\right\|_{\ell_{n_{b}}^{\infty}}=\left\|\sum_{\substack{\mathbf{n} \in \mathfrak{N}\left(\mathcal{T}_{1}\right) \\ n_{r}=n_{b}}} \prod_{a \in \mathcal{T}_{1}^{\infty}} \mathbf{u}_{n_{a}}\right\|_{\ell_{n_{b}}^{\infty}} \leqslant\left\|\mathcal{F}^{-1}\left(\left|\widehat{\mathbf{u}}_{n}\right|\right)\right\|_{L_{x}^{3}}^{3} \lesssim\|\mathbf{u}\|_{H^{1 / 6}}^{3} .
$$


- Case 1: We first consider the case $\Pi_{2}\left(\mathcal{T}_{J}\right)=\left\{r_{2}\right\}$.

Suppose that $b \neq r_{2}$ in (8.33). In this case, by summing over the $2 J$ variables $\left\{n_{a}\right\}_{a \in \mathcal{T}_{J}^{\infty} \backslash\left\{b, r_{2}\right\}}$ first and then over $n \in \mathbb{Z}$, we have

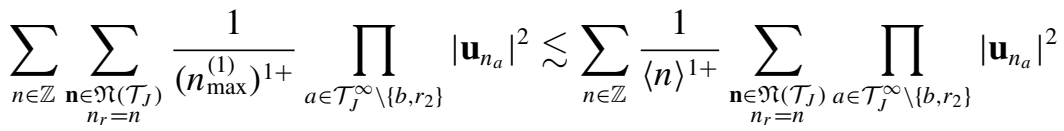

$$
\begin{aligned}
& \lesssim\|\mathbf{u}\|_{L^{2}}^{4 J} .
\end{aligned}
$$

Then, by Cauchy-Schwarz inequality with (8.22), (8.35), (8.36), and $\left|\mathcal{T}_{J} \backslash\left\{r_{2}\right\}\right|=$ $2 J+1$, we have

$$
\begin{aligned}
& \sum_{n \in \mathbb{Z}}\left|\mathcal{N}_{2}^{(J+1)}(\mathbf{u})_{n}\right| \lesssim \sum_{\substack{\mathcal{T}_{J} \in \mathfrak{B} \mathfrak{T}(J) \\
\Pi_{2}\left(\mathcal{T}_{J}\right)=\left\{r_{2}\right\}}} \sum_{\substack{\mathcal{T}_{J} \\
\mathcal{T}_{J}}} \sum_{n \in \mathbb{Z}}\left|\mathbf{u}_{n}\right| \sum_{\substack{\mathbf{n} \in \mathfrak{N}\left(\mathcal{T}_{J}\right) \\
n_{r}=n \\
\left|\phi_{1}\right|>K \\
\widetilde{\phi}_{j} \mid \gg(2 j+2)^{3} K^{1-\theta} \\
j=2, \ldots, J}}\left\|\mathrm{~N}(\mathbf{u})_{n_{b}}\right\|_{\ell_{n_{b}}} \\
& \times \frac{1}{\prod_{j=1}^{J}\left|\widetilde{\phi}_{j}\right|} \prod_{a \in \mathcal{T}_{J}^{\infty} \backslash\left\{b, r_{2}\right\}}\left|\mathbf{u}_{n_{a}}\right| \\
& \lesssim J\left\|\mathbf{u}_{n}\right\|_{\ell_{n}^{2}}\|\mathbf{u}\|_{H^{1 / 6}}^{3} \\
& \times \sum_{\substack{\mathcal{T}_{J} \in \mathfrak{B} \mathfrak{T}(J) \\
\Pi_{2}\left(\mathcal{T}_{J}\right)=\left\{r_{2}\right\}}}\left\{\sum_{n \in \mathbb{Z}}\left(\sum_{\substack{\mathbf{n} \in \mathfrak{N}\left(\mathcal{T}_{J}\right) \\
n_{r}=n \\
\left|\phi_{1}\right|>K}} \frac{1}{\prod_{j=1}^{J}\left|\widetilde{\phi}_{j}\right|} \prod_{a \in \mathcal{T}_{J}^{\infty} \backslash\left\{b, r_{2}\right\}}\left|\mathbf{u}_{n_{a}}\right|\right)^{2}\right\}^{1 / 2} \\
& \left|\widetilde{\phi}_{j}\right| \gg(2 j+2)^{3} K^{1-\theta} \\
& \ll \frac{J c_{J}}{\prod_{j=1}^{J}(2 j+2)^{3 / 2}} K^{-J(1-\theta) / 2}\|\mathbf{u}\|_{L^{2}}\|\mathbf{u}\|_{H^{1 / 6}}^{3}
\end{aligned}
$$

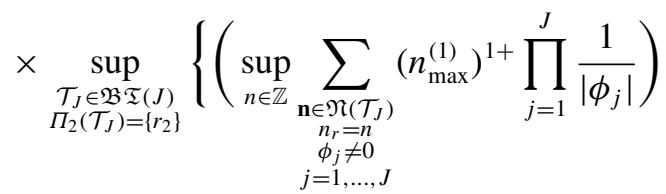

$$
\begin{aligned}
& \left.\times\left(\sum_{n \in \mathbb{Z}} \sum_{\substack{\mathbf{n} \in \mathfrak{N}\left(\mathcal{T}_{J}\right) \\
n_{r}=n}} \frac{1}{\left(n_{\max }^{(1)}\right)^{1+}} \prod_{\substack{a \in \mathcal{T}_{J}^{\infty} \backslash\left\{b, r_{2}\right\} \\
J \mathbf{u}^{\prime}}}\left|\mathbf{u}_{n_{a}}\right|^{2}\right)\right\}^{1 / 2} \\
& \lesssim \frac{J C^{J} c_{J}}{\prod_{j=1}^{J}(2 j+2)^{3 / 2}} K^{-J(1-\theta) / 2}\|\mathbf{u}\|_{L^{2}}^{2 J+1}\|\mathbf{u}\|_{H^{1 / 6}}^{3} \longrightarrow 0
\end{aligned}
$$

for any $K>0$, as $J \rightarrow \infty$. See Section 8.5 for a more precise condition on $K$ required for the convergence of the series for $\mathcal{N}_{0}^{(j)}, \mathcal{N}_{1}^{(j)}$, and $\mathcal{R}^{(j)}$. 
Next, suppose that $b=r_{2}$ in (8.33). This time, we need to work our way from the bottom. Let us first state and prove a useful lemma which follows from the nonresonance condition in Definition 8.3(c) and the divisor counting argument.

LEMMA 8.16. Let $J \in \mathbb{N}$. Given an ordered bi-tree $\mathcal{T}_{J} \in \mathfrak{B T}(J)$ with a chronicle $\left\{\mathcal{T}_{j}\right\}_{j=1}^{J}$ such that $\Pi_{2}\left(\mathcal{T}_{J}\right)=\left\{r_{2}\right\}$, fix a $\in \mathcal{T}_{J}^{\infty} \backslash \mathcal{T}_{J-1}^{\infty}$. (By convention, we set $\mathcal{T}_{0}$ to be a bi-tree of the zeroth generation consisting of the two root nodes $r_{1}$ and $r_{2}$ joined by an edge. Hence, we have $\mathcal{T}_{0}^{\infty}=\left\{r_{1}, r_{2}\right\}$.) Then, for fixed $m \in \mathbb{Z}$ and $v_{j} \in \mathbb{Z}, j=1, \ldots, J$, we have

$$
\#\left\{\mathbf{n} \in \mathfrak{N}\left(\mathcal{T}_{J}\right): n_{a}=m, \mu_{j}(\mathbf{n})=v_{j}, j=1, \ldots, J\right\} \leqslant C^{J} \prod_{j=1}^{J}\left|v_{j}\right|^{0+} .
$$

In view of $\Pi_{2}\left(\mathcal{T}_{J}\right)=\left\{r_{2}\right\}$, we can identify the ordered bi-tree $\mathcal{T}_{J}$ with an ordinary (ternary) ordered tree (in the sense of [18, Definition 3.3]). Lemma 8.16 is really a property of an ordered tree. Before proceeding to the proof of Lemma 8.16, let us recall the following arithmetic fact [20]. Given $n \in \mathbb{N}$, the number $d(n)$ of the divisors of $n$ satisfies

$$
d(n) \leqslant C_{\delta} n^{\delta}
$$

for any $\delta>0$.

Proof of Lemma 8.16. We first consider the case $J=1$. Let $r_{1 j}, j=1,2,3$ be the children of the first root node $r_{1}$. Then, it follows from $\mu_{1}=-2\left(n_{r_{12}}-n_{r_{11}}\right)\left(n_{r_{12}}-\right.$ $\left.n_{r_{13}}\right)$ and (8.39) that given $n_{r_{1 k}}=m$ for some $k \in\{1,2,3\}$, there are at most $o\left(\left|\mu_{1}\right|^{0+}\right)$ many choices for $n_{r_{1 j}}, j \neq k$ and hence for $n_{r}=n_{r_{1}}$.

When $J \geqslant 2$, (8.38) follows from an induction. In obtaining the ordered bi-tree $\mathcal{T}_{J}$, we replaced one of the terminal nodes, say $b \in \mathcal{T}_{J-1}^{\infty}$ into a nonterminal node. In particular, $a \in \mathcal{T}_{J}^{\infty} \backslash \mathcal{T}_{J-1}^{\infty}$ must be a child of $b$. Then, applying the argument for the $J=1$ case, we see that for fixed $n_{a}=m \in \mathbb{Z}$ and $\mu_{J} \in \mathbb{Z}$, there are at most $o\left(\left|\mu_{J}\right|^{0+}\right.$ ) many choices for $n_{b}$ (and the frequencies of the other two children of $b$ ). Now that we have fixed $n_{b}$ (up to $o\left(\left|\mu_{J}\right|^{0+}\right.$ ) many choices), (8.38) follows from the inductive hypothesis on $\mathcal{T}_{J-1}$.

REMARK 8.17. Note that Lemma 8.16 also holds even if we replace any of $\mu_{j}$ by $\phi_{j}$.

We continue with the proof of Lemma 8.15. Before proceeding to the case $b=r_{2}$, let us go over the main idea in the previous case $\left(b \neq r_{2}\right)$. When $b \neq r_{2}$, we placed $\mathrm{N}(\mathbf{u})_{n_{b}}$ in the $\ell_{n_{b}}^{\infty}$-norm and by expressing the summation over 
$\mathbf{n} \in \mathfrak{N}\left(\mathcal{T}_{J}\right)$ as

$$
\sum_{\mathbf{n} \in \mathfrak{N}\left(\mathcal{T}_{J}\right)}=\sum_{n \in \mathbb{Z}} \sum_{\substack{\mathbf{n} \in \mathfrak{N}\left(\mathcal{T}_{J}\right) \\ n_{r}=n}}
$$

we applied Cauchy-Schwarz inequality (in particular, in $n_{r}=n$ ) in the second inequality in (8.37), thus creating the factor

$$
\left\|\mathbf{u}_{n}\right\|_{\ell_{n}^{2}}\left\|\mathbf{N}(\mathbf{u})_{n_{b}}\right\|_{\ell_{n_{b}}^{\infty}} \lesssim\left\|\mathbf{u}_{n}\right\|_{\ell_{n}^{2}}\|\mathbf{u}\|_{H^{1 / 6}}^{3}
$$

thanks to (8.35). This left $2 J$ factors $\mathbf{u}_{n_{a}}, a \in \mathcal{T}_{J}^{\infty} \backslash\left\{b, r_{2}\right\}$, to which we applied (8.36). In this argument, it was crucial that we have $b \neq r_{2}$ so that we have the factor $\mathbf{u}_{n}=\mathbf{u}_{n_{r_{2}}}$ for the application of Cauchy-Schwarz inequality in $n_{r_{2}}=n$.

When $b=r_{2}$, we no longer have the factor $\mathbf{u}_{n}=\mathbf{u}_{n_{r_{2}}}$. Instead, the term corresponding to the frequency $n_{r_{2}}$ is given by $\mathrm{N}(\mathbf{u})_{n_{r_{2}}}$, which we place in the $\ell_{n_{r_{2}}}^{\infty}$-norm as in the previous case. Now, fix $\alpha \in \mathcal{T}_{J}^{\infty} \backslash \mathcal{T}_{J-1}^{\infty}$. Note that $\alpha \neq r_{2}$. Write

$$
\sum_{\mathbf{n} \in \mathfrak{N}\left(\mathcal{T}_{J}\right)}=\sum_{m \in \mathbb{Z}} \sum_{\substack{\mathbf{n} \in \mathfrak{N}\left(\mathcal{T}_{J}\right) \\ n_{\alpha}=m}}
$$

Namely, we single out the frequency $n_{\alpha}=m$ at the terminal node $\alpha \in \mathcal{T}_{J}^{\infty} \backslash$ $\mathcal{T}_{J-1}^{\infty}$. Compare this with (8.40) from the previous case, where we singled out the frequency $n_{r}=n$ at the terminal node $r_{2} \in \mathcal{T}_{J}^{\infty}$. In the following, we use $\mathbf{u}_{m}=\mathbf{u}_{n_{\alpha}}$ as a replacement of $\mathbf{u}_{n}=\mathbf{u}_{n_{r}}$ in the previous case and apply Cauchy-Schwarz inequality in $n_{\alpha}=m$. Also, note that, as a variant of (8.36), we have

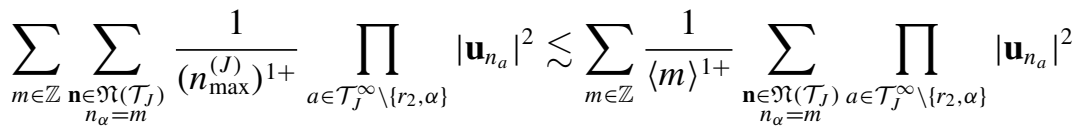

$$
\begin{aligned}
& \lesssim\|\mathbf{u}\|_{L^{2}}^{4 J} .
\end{aligned}
$$

Indeed, (8.42) follows from first summing over the $2 J$ variables $\left\{n_{a}\right\}_{a \in \mathcal{T}_{J}^{\infty} \backslash\left\{r_{2}, \alpha\right\}}$ and then over $m \in \mathbb{Z}$ with $n_{\max }^{(J)} \geqslant\left|n_{\alpha}\right|$. Then, from Cauchy-Schwarz inequality, (8.22), (8.35), (8.40), and (8.41), we have

$$
\begin{aligned}
\sum_{n \in \mathbb{Z}}\left|\mathcal{N}_{2}^{(J+1)}(\mathbf{u})_{n}\right| & \lesssim\left\|\mathrm{N}(\mathbf{u})_{n}\right\|_{\ell_{n}^{\infty}} \sum_{\substack{\mathcal{T}_{J} \in \mathfrak{B} \mathfrak{T}(J) \\
\Pi_{2}\left(\mathcal{T}_{J}\right)=\left\{r_{2}\right\}}} \sum_{\substack{\mathbf{n} \in \mathfrak{N}\left(\mathcal{T}_{J}\right) \\
\left|\phi_{1}\right|>K \\
\left|\tilde{\phi}_{j}\right| \gg(2 j+2)^{3} K^{1-\theta} \\
j=2, \ldots, J}} \frac{1}{\prod_{j=1}^{J}\left|\widetilde{\phi}_{j}\right|} \prod_{a \in \mathcal{T}_{J}^{\infty} \backslash\left\{r_{2}\right\}}\left|\mathbf{u}_{n_{a}}\right| \\
& \ll \frac{c_{J}}{\prod_{j=1}^{J}(2 j+2)^{3 / 2}} K^{-J(1-\theta) / 2}\|\mathbf{u}\|_{H^{1 / 6}}^{3}
\end{aligned}
$$




$$
\begin{aligned}
& \times \sup _{\substack{\mathcal{T}_{J} \in \mathfrak{B} \mathfrak{T}_{(J)} \\
\Pi_{2}\left(\mathcal{T}_{J}\right)=\left\{r_{2}\right\}}}\left\{\sum_{m \in \mathbb{Z}} \sum_{\substack{\mathbf{n} \in \mathfrak{N}\left(\mathcal{T}_{J}\right) \\
n_{\alpha}=m \\
\phi_{1}\left|>K \\
\tilde{\phi}_{j}\right| \gg(2 j+2)^{3} K^{1-\theta} \\
j=2, \ldots, J}}\left|\mathbf{u}_{n_{\alpha}}\right|^{2} \cdot\left(n_{\max }^{(J)}\right)^{1+} \prod_{j=1}^{J} \frac{1}{\left|\phi_{j}\right|}\right\}^{1 / 2} \\
& \times\left\{\sum_{\mathbf{n} \in \mathfrak{N}\left(\mathcal{T}_{J}\right)} \frac{1}{\left(n_{\max }^{(J)}\right)^{1+}} \prod_{a \in \mathcal{T}_{J}^{\infty} \backslash\left\{r_{2}, \alpha\right\}}\left|\mathbf{u}_{n_{a}}\right|^{2}\right\}^{1 / 2} .
\end{aligned}
$$

By (8.42), (8.4), and Lemma 8.16,

$$
\begin{aligned}
\lesssim & \frac{C^{J} c_{J}}{\prod_{j=1}^{J}(2 j+2)^{3 / 2}} K^{-J(1-\theta) / 2}\|\mathbf{u}\|_{L^{2}}^{2 J}\|\mathbf{u}\|_{H^{1 / 6}}^{3} \\
& \times \sup _{\substack{\mathcal{T}_{J} \in \mathfrak{B}^{2}(J) \\
\Pi_{2}\left(\mathcal{T}_{J}\right)=\left\{r_{2}\right\}}}\left\{\sum_{m \in \mathbb{Z}}\left|\mathbf{u}_{m}\right|^{2} \sum_{\substack{v_{j} \in \mathbb{Z} \backslash\{0\} \\
j=1, \ldots, J}} \frac{1}{\left|v_{J}\right|^{3 / 2}} \prod_{j=1}^{J-1} \frac{1}{\left|v_{j}\right|^{2-}}\right\}^{1 / 2} \\
\lesssim & \frac{C^{J} c_{J}}{\prod_{j=1}^{J}(2 j+2)^{3 / 2}} K^{-J(1-\theta) / 2}\|\mathbf{u}\|_{L^{2}}^{2 J+1}\|\mathbf{u}\|_{H^{1 / 6}}^{3} \longrightarrow 0,
\end{aligned}
$$

as $J \rightarrow \infty$.

- Case 2: Next, we consider the case $\Pi_{2}\left(\mathcal{T}_{J}\right) \neq\left\{r_{2}\right\}$. Note that we have $b \neq r_{2}$ by assumption. In this case, we can proceed as in (8.43) by replacing $r_{2}$ by $b$ and choosing $\alpha \in \mathcal{T}_{J}^{\infty} \backslash\left(\mathcal{T}_{J-1}^{\infty} \cup\{b\}\right)$.

REMARK 8.18. (i) If we assume a higher regularity $\mathbf{u} \in H^{\sigma}(\mathbb{T}), \sigma>\frac{1}{2}$, we can conclude (8.34) simply by the algebra property of $H^{\sigma}(\mathbb{T})$, which suffices for our purpose. See [33, Section 4.4]. We, however, decided to include the argument above since this provides the sharp regularity criterion $\left(\sigma \geqslant \frac{1}{6}\right)$ for the vanishing of the error term. Moreover, Lemma 8.16 seems to be of independent interest.

(ii) In view of the equation (2.2) with the cubic nonlinearity, we see $\sigma=\frac{1}{6}$ is the minimum regularity required for the application of the Leibniz rule in (8.9), (8.15), and (8.18). See [18]. By a computation similar to that in this subsection, we can also justify the switching of the time derivatives and the summations when $\sigma \geqslant \frac{1}{6}$ (by the dominated convergence theorem). We point out that it is also possible to justify the switching of the time derivatives and the summations as temporal distributions under a weaker assumption. See [18, Lemma 5.1]. 
8.5. Proof of Proposition 7.1. In this section, we put together all the estimates obtained in Sections 8.2-8.4 and prove Proposition 7.1.

Let $u$ be a smooth global solution to the Wick ordered cubic 4NLS (1.5) and $\mathbf{u}$ be its interaction representation as above. Then, by applying the normal form reductions $J$ times, we obtain

$$
\partial_{t}\left|\mathbf{u}_{n}\right|^{2}=\partial_{t} \sum_{j=2}^{J+1} \mathcal{N}_{0}^{(j)}(\mathbf{u})_{n}+\sum_{j=2}^{J+1} \mathcal{R}^{(j)}(\mathbf{u})_{n}+\sum_{j=1}^{J+1} \mathcal{N}_{1}^{(j)}(\mathbf{u})_{n}+\mathcal{N}_{2}^{(J+1)}(\mathbf{u})_{n}
$$

(Once again, we are replacing \pm 1 and $\pm i$ by 1 for simplicity since they play no role in our analysis.) In view of Lemma 8.15, by taking the limit as $J \rightarrow \infty$, we obtain

$$
\partial_{t}\left|\mathbf{u}_{n}\right|^{2}=\partial_{t} \sum_{j=2}^{\infty} \mathcal{N}_{0}^{(j)}(\mathbf{u})_{n}+\sum_{j=2}^{\infty} \mathcal{R}^{(j)}(\mathbf{u})_{n}+\sum_{j=1}^{\infty} \mathcal{N}_{1}^{(j)}(\mathbf{u})_{n} .
$$

Then, integration over $[0, t]$ yields the identity (7.2) for smooth solutions. (With a slight abuse of notations, we are identifying $\mathcal{N}_{0}^{(j)}(\mathbf{u})_{n}$ with $\mathcal{N}_{0}^{(j)}(u)_{n}$, and so on. The same comment applies in the following.) Furthermore, the multilinear estimates (7.3), (7.4), and (7.5) follow from Lemmas 8.5, 8.8, 8.9, 8.10, 8.12, 8.13, and 8.14 and choosing $\theta \in\left(0, \frac{2}{3}\right]$. (We fix an absolute constant $\theta \in\left(0, \frac{2}{3}\right]$ once and for all and thus suppress dependence of various constants on $\theta$ in the following.)

In the following, we verify (7.2)-(7.5) for rough solutions $u$ to (1.5) by an approximation argument. Fix $s \in\left(-\frac{1}{3}, 0\right)$. Let $u$ be a (possibly nonunique) solution to (1.5) in $C\left([-T, T] ; H^{s}(\mathbb{T})\right) \cap F^{s, \alpha}(T)$, that is, with $M=1$, constructed in Section 6. Note that we have $T=T\left(\|u(0)\|_{H^{s}}\right)>0$. See Remark 6.4.

Let $u_{N}$ be a smooth solution to (1.5) with $\left.u_{N}\right|_{t=0}=\mathbf{P}_{\leqslant N} u(0)$. Then, from the construction in Section 6, there exists a subsequence $\left\{u_{N_{k}}\right\}_{k \in \mathbb{N}}$ such that

$$
\lim _{k \rightarrow \infty}\left\|u-u_{N_{k}}\right\|_{C_{T} H^{s}}=0 .
$$

Moreover, $u_{N_{k}}$ and $u$ satisfy a uniform bound:

$$
\sup _{k \in \mathbb{N}}\left\|u_{N_{k}}\right\|_{C_{T} H^{s}},\|u\|_{C_{T} H^{s}} \leqslant r \sim\|u(0)\|_{H^{s}} .
$$

Hence, it follows from (8.44) and (8.45) that, for each fixed $n \in \mathbb{Z}$, we have

$$
\lim _{k \rightarrow \infty}\left\{\left|\widehat{u}_{N_{k}}(n, t)\right|^{2}-\left|\widehat{u}_{N_{k}}(n, 0)\right|^{2}\right\}=|\widehat{u}(n, t)|^{2}-|\widehat{u}(n, 0)|^{2},
$$

uniformly in $t \in[-T, T]$. 
Since $u_{N_{k}}$ is smooth, we have

$$
\begin{aligned}
\left|\widehat{u}_{N_{k}}(n, t)\right|^{2}- & \left|\widehat{u}_{N_{k}}(n, 0)\right|^{2} \\
= & \left.\sum_{j=2}^{\infty} \mathcal{N}_{0}^{(j)}\left(u_{N_{k}}\right)\left(n, t^{\prime}\right)\right|_{0} ^{t} \\
& +\int_{0}^{t}\left[\sum_{j=1}^{\infty} \mathcal{N}_{1}^{(j)}\left(u_{N_{k}}\right)\left(n, t^{\prime}\right)+\sum_{j=2}^{\infty} \mathcal{R}^{(j)}\left(u_{N_{k}}\right)\left(n, t^{\prime}\right)\right] d t^{\prime} .
\end{aligned}
$$

Note that the identity (7.2) for a rough solution $u$ follows from (8.46) and (8.47) once we prove

$$
\begin{gathered}
\lim _{k \rightarrow \infty} \sum_{j=2}^{\infty} \mathcal{N}_{0}^{(j)}\left(u_{N_{k}}\right)(n, t)=\sum_{j=2}^{\infty} \mathcal{N}_{0}^{(j)}(u)(n, t), \\
\lim _{k \rightarrow \infty} \int_{0}^{t} \sum_{j=2}^{\infty} \mathcal{R}^{(j)}\left(u_{N_{k}}\right)\left(n, t^{\prime}\right) d t^{\prime}=\int_{0}^{t} \sum_{j=2}^{\infty} \mathcal{R}^{(j)}(u)\left(n, t^{\prime}\right) d t^{\prime}, \\
\lim _{k \rightarrow \infty} \int_{0}^{t} \sum_{j=1}^{\infty} \mathcal{N}_{1}^{(j)}\left(u_{N_{k}}\right)\left(n, t^{\prime}\right) d t^{\prime}=\int_{0}^{t} \sum_{j=1}^{\infty} \mathcal{N}_{1}^{(j)}(u)\left(n, t^{\prime}\right) d t^{\prime},
\end{gathered}
$$

uniformly in $t \in[-T, T]$. In the following, we only verify (8.48), since (8.49) and (8.50) follow in an analogous manner.

From Lemmas 8.8 and 8.12 with the multilinearity of $\mathcal{N}_{0}^{(j)}$ and (8.45), we can choose $K=K(r) \gg 1$ such that

$$
\begin{aligned}
& \left|\sum_{j=2}^{\infty} \mathcal{N}_{0}^{(j)}\left(u_{N_{k}}\right)(n, t)-\sum_{j=2}^{\infty} \mathcal{N}_{0}^{(j)}(u)(n, t)\right| \\
& \quad \lesssim K^{\max (-1 / 2,-1-2 s)} r^{3}\left\|u-u_{N_{k}}\right\|_{C_{T} H^{s}}+\sum_{j=3}^{\infty} K^{-((j-1)(1-\theta)) / 2} r^{2 j+1}\left\|u-u_{N_{k}}\right\|_{C_{T} H^{s}} \\
& \quad \lesssim C(r)\left\|u-u_{N_{k}}\right\|_{C_{T} H^{s}} \longrightarrow 0,
\end{aligned}
$$

as $k \rightarrow \infty$, uniformly in $t \in[-T, T]$. This proves (8.48).

Finally, note that, in view of the global existence (Theorem 1.1) and the Sobolev norm bound (Proposition 6.6), we can iterate the above discussion to conclude the identity (7.2) for all $t \in \mathbb{R}$. This completes the proof of Proposition 7.1.

REMARK 8.19. In the above argument, we assumed that $u \in C([-T$, $\left.T] ; H^{s}(\mathbb{T})\right) \cap F^{s, \alpha}(T)$. It is, however, sufficient to assume that $u \in C([-T$, $\left.T] ; H^{s}(\mathbb{T})\right)$ is a solution to (2.2) for some $T=T\left(\left\|u_{0}\right\|_{H^{s}}\right)>0$ with some smooth 
approximating solutions $\left\{u_{n}\right\}_{n \in \mathbb{N}}$ such that

$$
\lim _{n \rightarrow \infty}\left\|u-u_{n}\right\|_{C_{T} H^{s}}=0 \quad \text { and } \quad \sup _{n \in \mathbb{N}}\left\|u_{n}\right\|_{C_{T} H^{s}},\|u\|_{C_{T} H^{s}} \lesssim\|u(0)\|_{H^{s}},
$$

replacing (8.44) and (8.45). The same comment applies to the proof of Proposition 7.2 presented in the next subsection.

8.6. Energy estimate for the nonresonant part. In this subsection, we briefly discuss the proof of Proposition 7.2 on the nonresonant part of the energy estimate (7.1) for the difference of two solutions with the same initial condition. In fact, we reduce the matter to (a slight modification of) the discussion in the previous subsections.

Given $u_{0} \in H^{s}(\mathbb{T}), s>-\frac{1}{3}$, let $u$ and $v$ be two solutions to (1.5) on $[-T, T]$ with the same initial condition $\left.u\right|_{t=0}=\left.v\right|_{t=0}=u_{0}$, satisfying

$$
\|u\|_{C_{T} H^{s}},\|v\|_{C_{T} H^{s}} \leqslant r \sim\left\|u_{0}\right\|_{H^{s}} .
$$

Let $\mathbf{u}$ and $\mathbf{v}$ denote the interaction representations of $u$ and $v$, respectively. Then, from (7.1), we have

$$
\begin{aligned}
\mathrm{I}= & -2 \operatorname{Re} i \sum_{n \in \mathbb{Z}}\langle n\rangle^{2 s}\left[{\widehat{\mathrm{N}(\mathbf{u})_{n}}}_{n}-\widehat{\left.\mathrm{N}(\mathbf{v})_{n}\right]} \overline{\left(\widehat{\mathbf{u}}_{n}-\widehat{\mathbf{v}}_{n}\right)}\right. \\
= & -2 \operatorname{Re} i \sum_{n \in \mathbb{Z}}\langle n\rangle^{2 s} \widehat{\mathrm{N}(\mathbf{u})_{n}} \widehat{\mathbf{u}}_{n}+2 \operatorname{Re} i \sum_{n \in \mathbb{Z}}\langle n\rangle^{2 s} \widehat{\mathrm{N}(\mathbf{u})_{n}} \widehat{\mathbf{v}}_{n} \\
& +2 \operatorname{Re} i \sum_{n \in \mathbb{Z}}\langle n\rangle^{2 s} \widehat{\mathrm{N}(\mathbf{v})_{n}} \widehat{\mathbf{u}}_{n}-2 \operatorname{Re} i \sum_{n \in \mathbb{Z}}\langle n\rangle^{2 s} \widehat{\mathrm{N}(\mathbf{v})_{n}} \widehat{\mathbf{v}}_{n} \\
= & : \mathrm{I}_{u u}-\mathrm{I}_{u v}-\mathrm{I}_{v u}+\mathrm{I}_{v v} .
\end{aligned}
$$

From (8.5) with (2.2), we have

$$
\mathrm{I}_{u u}=\sum_{n \in \mathbb{Z}}\langle n\rangle^{2 s} \mathcal{N}^{(1)}(\mathbf{u})_{n} \quad \text { and } \quad \mathrm{I}_{v v}=\sum_{n \in \mathbb{Z}}\langle n\rangle^{2 s} \mathcal{N}^{(1)}(\mathbf{v})_{n} .
$$

By repeating the arguments in the previous subsections, we have

$$
\begin{aligned}
& \int_{0}^{t} \mathrm{I}_{u u}\left(t^{\prime}\right) d t^{\prime}=\left.\sum_{j=2}^{\infty} \sum_{n \in \mathbb{Z}}\langle n\rangle^{2 s} \mathcal{N}_{0}^{(j)}(\mathbf{u})\left(n, t^{\prime}\right)\right|_{0} ^{t} \\
& +\int_{0}^{t}\left[\sum_{j=2}^{\infty} \sum_{n \in \mathbb{Z}}\langle n\rangle^{2 s} \mathcal{R}^{(j)}(\mathbf{u})\left(n, t^{\prime}\right)+\sum_{j=1}^{\infty} \sum_{n \in \mathbb{Z}}\langle n\rangle^{2 s} \mathcal{N}_{1}^{(j)}(\mathbf{u})\left(n, t^{\prime}\right)\right] d t^{\prime}
\end{aligned}
$$


and

$$
\begin{aligned}
& \int_{0}^{t} \mathrm{I}_{v v}\left(t^{\prime}\right) d t^{\prime}=\left.\sum_{j=2}^{\infty} \sum_{n \in \mathbb{Z}}\langle n\rangle^{2 s} \mathcal{N}_{0}^{(j)}(\mathbf{v})\left(n, t^{\prime}\right)\right|_{0} ^{t} \\
& +\int_{0}^{t}\left[\sum_{j=2}^{\infty} \sum_{n \in \mathbb{Z}}\langle n\rangle^{2 s} \mathcal{R}^{(j)}(\mathbf{v})\left(n, t^{\prime}\right)+\sum_{j=1}^{\infty} \sum_{n \in \mathbb{Z}}\langle n\rangle^{2 s} \mathcal{N}_{1}^{(j)}(\mathbf{v})\left(n, t^{\prime}\right)\right] d t^{\prime}
\end{aligned}
$$

In Sections 8.2 and 8.3, we performed the normal form reductions for each fixed $n \in \mathbb{Z}$ and the weight $\langle n\rangle^{2 s}$ in (8.52) does not affect the argument.

In order to handle the cross terms $\mathrm{I}_{u v}$ and $\mathrm{I}_{v u}$, we need to introduce new notations. Define $\tilde{\mathcal{N}}^{(1)}(\mathbf{u}, \mathbf{v})_{n}$ by

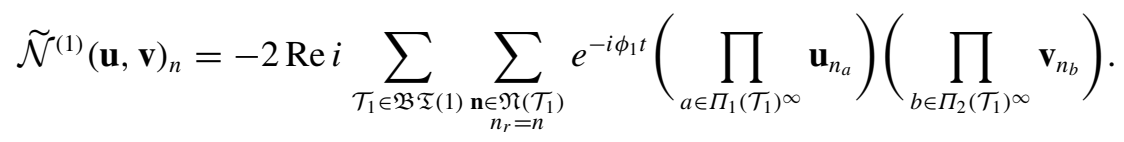

Namely, $\tilde{\mathcal{N}}^{(1)}(\mathbf{u}, \mathbf{v})_{n}$ is constructed from $\mathcal{N}_{n}^{(1)}$ in (8.5) by taking different functions $\mathbf{u}$ and $\mathbf{v}$ over the terminal nodes of the first tree $\Pi_{1}\left(\mathcal{T}_{1}\right)$ and the second tree $\Pi_{2}\left(\mathcal{T}_{1}\right)$, respectively. (Note that the second tree $\Pi_{2}\left(\mathcal{T}_{1}\right)$ consists only of the (second) root node $r_{2}$. We, however, use this notation in order to be consistent with the general case. See (8.55), (8.56), and (8.57).)

Then, we have

$$
\mathrm{I}_{u v}=\sum_{n \in \mathbb{Z}}\langle n\rangle^{2 s} \tilde{\mathcal{N}}^{(1)}(\mathbf{u}, \mathbf{v})_{n} \quad \text { and } \quad \mathrm{I}_{v u}=\sum_{n \in \mathbb{Z}}\langle n\rangle^{2 s} \tilde{\mathcal{N}}^{(1)}(\mathbf{v}, \mathbf{u})_{n} .
$$

We also make similar modifications to the multilinear terms introduced in Sections 8.2 and 8.3 and define $\widetilde{\mathcal{N}}_{0}^{(j)}(\mathbf{u}, \mathbf{v})_{n}, \widetilde{\mathcal{R}}^{(j)}(\mathbf{u}, \mathbf{v})_{n}$, and $\widetilde{\mathcal{N}}^{(j)}(\mathbf{u}, \mathbf{v})_{n}$ by

$$
\begin{aligned}
\tilde{\mathcal{N}}_{0}^{(j)}(\mathbf{u}, \mathbf{v})_{n}:= & \sum_{\mathcal{T}_{j-1} \in \mathfrak{B} \mathfrak{T}(j-1)} \sum_{\mathbf{n} \in \mathfrak{N}\left(\mathcal{T}_{j-1}\right)} \frac{e^{-i \widetilde{\phi}_{j-1} t}}{\prod_{r=n}^{j-1} \widetilde{\phi}_{k}} \\
& \times\left(\prod_{a \in \Pi_{1}\left(\mathcal{T}_{j-1}\right)^{\infty}} \mathbf{u}_{n_{a}}\right)\left(\prod_{b \in \Pi_{2}\left(\mathcal{T}_{j-1}\right)^{\infty}} \mathbf{v}_{n_{b}}\right), \\
\widetilde{\mathcal{R}}^{(j)}(\mathbf{u}, \mathbf{v})_{n}:= & \sum_{\mathcal{T}_{j-1} \in \mathfrak{B} \mathfrak{T}(j-1)} \sum_{\alpha \in \mathcal{T}_{j-1}^{\infty}} \sum_{\mathbf{n} \in \mathfrak{N}\left(\mathcal{T}_{j-1}\right)} \frac{e^{-i \widetilde{\phi}_{j-1} t}}{\prod_{k=1}^{j-1} \widetilde{\phi}_{k}} \mathrm{R}(\mathbf{u})_{n_{\alpha}} \\
& \times\left(\prod_{a \in \Pi_{1}\left(\mathcal{T}_{j-1}\right)^{\infty} \backslash\{\alpha\}} \mathbf{u}_{n_{a}}\right)\left(\prod_{b \in \Pi_{2}\left(\mathcal{T}_{j-1}\right)^{\infty} \backslash\{\alpha\}} \mathbf{v}_{n_{b}}\right),
\end{aligned}
$$




$$
\tilde{\mathcal{N}}^{(j)}(\mathbf{u}, \mathbf{v})_{n}:=\sum_{\mathcal{T}_{j} \in \mathfrak{B} \mathfrak{T}(j)} \sum_{\substack{\mathbf{n} \in \mathfrak{N}\left(\mathcal{T}_{j}\right) \\ n_{r}=n}} \frac{e^{-i \tilde{\phi}_{j} t}}{\prod_{k=1}^{j-1} \widetilde{\phi}_{k}}\left(\prod_{a \in \Pi_{1}\left(\mathcal{T}_{j}\right)^{\infty}} \mathbf{u}_{n_{a}}\right)\left(\prod_{b \in \Pi_{2}\left(\mathcal{T}_{j}\right)^{\infty}} \mathbf{v}_{n_{b}}\right) .
$$

(As mentioned in Remark 8.7, we are dropping unimportant, $\pm \pm i$, and 2 Re.) Compare these definitions with (8.18). Moreover, we define $\tilde{\mathcal{N}}_{1}^{(j)}(\mathbf{u}, \mathbf{v})_{n}$ and $\tilde{\mathcal{N}}_{2}^{(j)}(\mathbf{u}, \mathbf{v})_{n}$ as the restrictions of $\tilde{\mathcal{N}}^{(j)}(\mathbf{u}, \mathbf{v})_{n}$ onto $C_{j-1}$ and $C_{j-1}^{c}$ (see (8.30)). Then, from the discussion in the previous subsections, we have

$$
\begin{aligned}
\int_{0}^{t} \mathrm{I}_{u v}\left(t^{\prime}\right) d t^{\prime}= & \left.\sum_{j=2}^{\infty} \sum_{n \in \mathbb{Z}}\langle n\rangle^{2 s} \widetilde{\mathcal{N}}_{0}^{(j)}(\mathbf{u}, \mathbf{v})\left(n, t^{\prime}\right)\right|_{0} ^{t} \\
& +\int_{0}^{t}\left[\sum_{j=2}^{\infty} \sum_{n \in \mathbb{Z}}\langle n\rangle^{2 s} \widetilde{\mathcal{R}}^{(j)}(\mathbf{u}, \mathbf{v})\left(n, t^{\prime}\right)\right. \\
& \left.+\sum_{j=1}^{\infty} \sum_{n \in \mathbb{Z}}\langle n\rangle^{2 s} \tilde{\mathcal{N}}_{1}^{(j)}(\mathbf{u}, \mathbf{v})\left(n, t^{\prime}\right)\right] d t^{\prime}
\end{aligned}
$$

and

$$
\begin{aligned}
\int_{0}^{t} \mathrm{I}_{v u}\left(t^{\prime}\right) d t^{\prime}= & \left.\sum_{j=2}^{\infty} \sum_{n \in \mathbb{Z}}\langle n\rangle^{2 s} \tilde{\mathcal{N}}_{0}^{(j)}(\mathbf{v}, \mathbf{u})\left(n, t^{\prime}\right)\right|_{0} ^{t} \\
& +\int_{0}^{t}\left[\sum_{j=2}^{\infty} \sum_{n \in \mathbb{Z}}\langle n\rangle^{2 s} \widetilde{\mathcal{R}}^{(j)}(\mathbf{v}, \mathbf{u})\left(n, t^{\prime}\right)\right. \\
& \left.+\sum_{j=1}^{\infty} \sum_{n \in \mathbb{Z}}\langle n\rangle^{2 s} \tilde{\mathcal{N}}_{1}^{(j)}(\mathbf{v}, \mathbf{u})\left(n, t^{\prime}\right)\right] d t^{\prime}
\end{aligned}
$$

In the following, we simply drop the factor $\langle n\rangle^{2 s}$. (By making use of the factor $\langle n\rangle^{2 s}$, we may extend Proposition 7.2 to $s>-\frac{1}{2}$. This, however, involves modifications of the multilinear estimates in Sections 8.2 and 8.3. In view of the regularity restriction $s>-\frac{1}{3}$ for the resonant part (Proposition 7.1), we simply use the multilinear estimates from Sections 8.2 and 8.3 and prove Proposition 7.2 for $s>-\frac{1}{3}$.) Then, by applying the multilinear estimates in Lemmas 8.5, 8.8, 8.9, 8.10, 8.12, 8.13, and 8.14 (with $\theta \in\left(0, \frac{2}{3}\right]$ ), it follows from (8.51), (8.53), (8.54), (8.58), and (8.59) that

$$
\begin{aligned}
& \left|\int_{0}^{t} \mathrm{I}\left(t^{\prime}\right) d t^{\prime}\right|=\left|\int_{0}^{t}\left\{\left(\mathrm{I}_{u u}\left(t^{\prime}\right)-\mathrm{I}_{u v}\left(t^{\prime}\right)\right)-\left(\mathrm{I}_{v u}\left(t^{\prime}\right)-\mathrm{I}_{v v}\left(t^{\prime}\right)\right)\right\} d t^{\prime}\right| \\
& \quad \lesssim K^{\max (-1 / 2,-1-2 s)} r^{2}\|u-v\|_{C_{T} H^{s}}^{2}+\sum_{j=3}^{\infty} K^{-((j-1)(1-\theta)) / 2} r^{2 j-2}\|u-v\|_{C_{T} H^{s}}^{2}
\end{aligned}
$$




$$
\begin{aligned}
& +T K^{\max (-1 / 2,-1-3 s)} r^{4}\|u-v\|_{C_{T} H^{s}}^{2}+T \sum_{j=3}^{\infty} K^{-((j-3)(1-\theta)) / 2} r^{2 j}\|u-v\|_{C_{T} H^{s}}^{2} \\
& +T K^{1 / 2-2 s} r^{2}\|u-v\|_{C_{T} H^{s}}^{2}+T \sum_{j=2}^{\infty} K^{-((j-2)(1-\theta)) / 2} r^{2 j}\|u-v\|_{C_{T} H^{s}}^{2},
\end{aligned}
$$

uniformly for $t \in[-T, T]$. Note that we obtained two factors of $u-v$ thanks to the double difference structure of (8.51). (By writing the double differences of the multilinear terms of the $j$ th generation in a telescoping sum, we obtain $O\left(j^{2}\right)$ many terms. This loss of $O\left(j^{2}\right)$ does not cause any issue in view of the fast decay in $j$ in the multilinear estimates in Section 8.3.) Then, by first choosing $K=K(r)>0$ sufficiently large and then choosing $T=T(K)=T(r)>0$ sufficiently small, we conclude that

$$
\left|\int_{0}^{t} \mathrm{I}\left(t^{\prime}\right) d t^{\prime}\right| \leqslant \frac{1}{4}\|u-v\|_{C_{T} H^{s}}^{2}
$$

for $t \in[-T, T]$. This completes the proof of Proposition 7.2.

REMARK 8.20. Integrating (7.1) from 0 to $t$, we obtain the identity:

$$
\begin{aligned}
\| u(t)- & v(t) \|_{H^{s}}^{2} \\
= & \int_{0}^{t}\left\{\left(\mathrm{I}_{u u}\left(t^{\prime}\right)-\mathrm{I}_{u v}\left(t^{\prime}\right)\right)-\left(\mathrm{I}_{v u}\left(t^{\prime}\right)-\mathrm{I}_{v v}\left(t^{\prime}\right)\right)\right\} d t^{\prime} \\
& +\int_{0}^{t} \sum_{n \in \mathbb{Z}}\langle n\rangle^{2 s}\left(\mathfrak{S}_{\infty}(u)\left(n, t^{\prime}\right)-\mathfrak{S}_{\infty}(v)\left(n, t^{\prime}\right)\right) \overline{\left(\widehat{u}_{n}-\widehat{v}_{n}\right)} \widehat{v}_{n}\left(t^{\prime}\right) d t^{\prime},
\end{aligned}
$$

where $\mathfrak{S}_{\infty}(u)(n, t)=\left|\widehat{u}_{n}(t)\right|^{2}-\left|\widehat{u}_{n}(0)\right|^{2}$ as in (1.14). In view of (7.2), (8.53), (8.54), (8.58), and (8.59), we see that both the first and second terms on the righthand side of (8.61) can be expressed as a sum of infinite series consisting of multilinear terms of increasing degrees. Furthermore, thanks to the multilinearity of the summands, we can extract two factors of (the Fourier coefficient of) $u-v$ in both the first and second terms on the right-hand side of (8.61).

In this paper, we established spatial multilinear estimates (for fixed $t$ ) and showed that these multilinear terms are summable, provided $s>-\frac{1}{3}$. This allows us to obtain the enhanced uniqueness in Theorem 1.5. It may be of interest to establish space-time estimates on these multilinear terms (arising from the energy of the difference of two solutions in the $E^{s}(T)$-norm rather than the $C_{T} H^{s}$-norm), namely in terms of the $F^{s, \alpha}(T)$-norm as in Section 5 , possibly allowing us to go 
below $s=-\frac{1}{3}$. We point out that the argument in [14] may be of use in estimating multilinear terms of (arbitrarily) large degrees.

REMARK 8.21. If we proceed with an energy estimate in the spirit of Proposition 5.2 in Section 5 (but without symmetrization) in terms of the $F^{s, \alpha}(T)$-norm, we can establish the following energy bound; let $s \in\left(-\frac{3}{10}, 0\right)$ and $\alpha=-8 s / 3+\varepsilon$ as in (4.12). Then, there exists $\theta>0$ such that

$$
\left|\int_{0}^{T} \mathrm{I}(t) d t\right| \lesssim T^{\theta}\left(\|u\|_{F^{s, \alpha}(T)}^{2}+\|v\|_{F^{s, \alpha}(T)}^{2}\right)\|u-v\|_{F^{s, \alpha}(T)}^{2}
$$

for any $T \in(0,1]$. By combining with the linear and nonlinear estimates (Lemma 2.5 and Proposition 4.1), the energy estimate (8.62) yields uniqueness for $s>-\frac{3}{10}$, not sufficient for Theorem 1.5.

\section{Acknowledgements}

T.O. and Y.W. were supported by the European Research Council (grant no. 637995 'ProbDynDispEq'). The authors would like to thank the anonymous referee for the helpful comments which improved the presentation of the paper.

\section{References}

[1] A. Babin, A. Ilyin and E. Titi, 'On the regularization mechanism for the periodic Korteweg-de Vries equation', Comm. Pure Appl. Math. 64(5) (2011), 591-648.

[2] M. Ben-Artzi, H. Koch and J. C. Saut, 'Dispersion estimates for fourth order Schrödinger equations', C. R. Acad. Sci. Paris Sér. I Math. 330(2) (2000), 87-92.

[3] J. Bourgain, 'Fourier transform restriction phenomena for certain lattice subsets and applications to nonlinear evolution equations I. Schrödinger equations', Geom. Funct. Anal. 3(2) (1993), 107-156.

[4] J. Bourgain, 'Invariant measures for the 2D-defocusing nonlinear Schrödinger equation', Comm. Math. Phys. 176(2) (1996), 421-445.

[5] N. Burq, P. Gérard and N. Tzvetkov, 'An instability property of the nonlinear Schrödinger equation on $\mathbb{S}^{d}$, Math. Res. Lett. 9(2-3) (2002), 323-335.

[6] A. Choffrut and O. Pocovnicu, 'Ill-posedness for the cubic nonlinear half-wave equation and other fractional NLS on the real line', Int. Math. Res. Not. IMRN 2018(3) 699-738.

[7] M. Christ, 'Nonuniqueness of weak solutions of the nonlinear Schrödinger equation', Preprint, 2005, arXiv:math/0503366v1 [math.AP].

[8] M. Christ, J. Colliander and T. Tao, 'Asymptotics, frequency modulation, and low regularity ill-posedness for canonical defocusing equations', Amer. J. Math. 125(6) (2003), 1235-1293.

[9] M. Christ, J. Colliander and T. Tao, 'Ill-posedness for nonlinear Schrödinger and wave equations', Preprint, 2003, arXiv:math/0311048 [math.AP].

[10] M. Christ, J. Colliander and T. Tao, 'A priori bounds and weak solutions for the nonlinear Schrödinger equation in Sobolev spaces of negative order', J. Funct. Anal. 254(2) (2008), 368-395. 
[11] J. Chung, Z. Guo, S. Kwon and T. Oh, 'Normal form approach to global well-posedness of the quadratic derivative Schrödinger equation on the circle', Ann. Inst. H. Poincaré Anal. Non Linéaire 34 (2017), 1273-1297.

[12] J. Colliander, M. Keel, G. Staffilani, H. Takaoka and T. Tao, 'A refined global well-posedness result for Schrödinger equations with derivative', SIAM J. Math. Anal. 34(1) (2002), 64-86.

[13] J. Colliander, M. Keel, G. Staffilani, H. Takaoka and T. Tao, 'Sharp global well-posedness for $\mathrm{KdV}$ and modified KdV on $\mathbb{R}$ and T', J. Amer. Math. Soc. 16(3) (2003), 705-749.

[14] J. Colliander, M. Keel, G. Staffilani, H. Takaoka and T. Tao, 'Multilinear estimates for periodic KdV equations, and applications', J. Funct. Anal. 211(1) (2004), 173-218.

[15] J. Colliander and T. Oh, 'Almost sure well-posedness of the periodic cubic nonlinear Schrödinger equation below $L^{2}(\mathbb{T})$ ', Duke Math. J. 161(3) (2012), 367-414.

[16] G. Fibich, B. Ilan and G. Papanicolaou, 'Self-focusing with fourth-order dispersion', SIAM J. Appl. Math. 62(4) (2002), 1437-1462.

[17] Z. Guo, 'Local well-posedness and a priori bounds for the modified Benjamin-Ono equation', Adv. Differential Equations 16(11-12) (2011), 1087-1137.

[18] Z. Guo, S. Kwon and T. Oh, 'Poincaré-Dulac normal form reduction for unconditional wellposedness of the periodic cubic NLS', Comm. Math. Phys. 322(1) (2013), 19-48.

[19] Z. Guo and T. Oh, 'Non-existence of solutions for the periodic cubic NLS below $L^{2}$, Int. Math. Res. Not. IMRN (6) (2018), 1656-1729.

[20] G. H. Hardy and E. M. Wright, An Introduction to the Theory of Numbers, 5th edn (The Clarendon Press, Oxford University Press, New York, 1979), xvi+426 pp.

[21] A. Ionescu, C. Kenig and D. Tataru, 'Global well-posedness of the KP-I initial-value problem in the energy space', Invent. Math. 173(2) (2008), 265-304.

[22] B. A. Ivanov and A. M. Kosevich, 'Stable three-dimensional small-amplitude soliton in magnetic materials', So. J. Low Temp. Phys. 9 (1983), 439-442.

[23] V. I. Karpman, 'Stabilization of soliton instabilities by higher-order dispersion: fourth order nonlinear Schrödinger-type equations', Phys. Rev. E 53(2) (1996), 1336-1339.

[24] V. I. Karpman and A. G. Shagalov, 'Solitons and their stability in high dispersive systems. I. Fourth-order nonlinear Schrödinger-type equations with power-law nonlinearities', Phys. Lett. A 228(1-2) (1997), 59-65.

[25] C. Kenig and D. Pilod, 'Well-posedness for the fifth-order KdV equation in the energy space', Trans. Amer. Math. Soc. 367(4) (2015), 2551-2612.

[26] H. Koch and D. Tataru, 'A priori bounds for the 1D cubic NLS in negative Sobolev spaces', Int. Math. Res. Not. IMRN 2007(16), Art. ID rnm053, 36 pp.

[27] H. Koch and D. Tataru, 'Energy and local energy bounds for the 1-d cubic NLS equation in $H^{-1 / 4}$, Ann. Inst. H. Poincaré Anal. Non Linéaire 29(6) (2012), 955-988.

[28] H. Koch and N. Tzvetkov, 'On the local well-posedness of the Benjamin-Ono equation in $H^{s}(\mathbb{R})$ ', Int. Math. Res. Not. IMRN 2003(26) (2003), 1449-1464.

[29] C. Kwak, 'Periodic fourth-order cubic NLS: Local well-posedness and Non-squeezing property', J. Math. Anal. Appl. 461(2) (2018), 1327-1364.

[30] T. Miyaji and Y. Tsutsumi, 'Local well-posedness of the NLS equation with third order dispersion in negative Sobolev spaces', Differential Integral Equations 31(1-2) (2018), 111-132.

[31] L. Molinet, D. Pilod and S. Vento, 'On unconditional well-posedness for the periodic modified Korteweg-de Vries equation', J. Math. Soc. Japan, to appear.

[32] K. Nakanishi, H. Takaoka and Y. Tsutsumi, 'Local well-posedness in low regularity of the mKdV equation with periodic boundary condition', Discrete Contin. Dyn. Syst. 28(4) (2010), 1635-1654. 
[33] T. Oh, P. Sosoe and N. Tzvetkov, 'An optimal regularity result on the quasi-invariant Gaussian measures for the cubic fourth order nonlinear Schrödinger equation', Preprint, 2017, arXiv:1 707.01666 [math.AP].

[34] T. Oh and C. Sulem, 'On the one-dimensional cubic nonlinear Schrödinger equation below $L^{2}$, Kyoto J. Math. 52(1) (2012), 99-115.

[35] T. Oh and L. Thomann, 'A pedestrian approach to the invariant Gibbs measure for the 2d defocusing nonlinear Schrödinger equations', Stoch. Partial Differ. Equ. Anal. Comput. (2018) https://doi.org/10.1007/s40072-018-0112-2.

[36] T. Oh and N. Tzvetkov, 'Quasi-invariant Gaussian measures for the cubic fourth order nonlinear Schrödinger equation', Probab. Theory Related Fields 169 (2017), 1121-1168.

[37] T. Oh, N. Tzvetkov and Y. Wang, Solving the 4NLS with white noise initial data, Preprint.

[38] T. Oh and Y. Wang, 'On the ill-posedness of the cubic nonlinear Schrödinger equation on the circle', An. Ştiinţ. Univ. Al. I. Cuza Iaşi. Mat. (N.S.), to appear.

[39] H. Takaoka and Y. Tsutsumi, 'Well-posedness of the Cauchy problem for the modified KdV equation with periodic boundary condition', Int. Math. Res. Not. IMRN 2004(56) (2004), 3009-3040.

[40] S. K. Turitsyn, 'Three-dimensional dispersion of nonlinearity and stability of multidimensional solitons', Teoret. Mat. Fiz. 64 (1985), 226-232 (in Russian). 